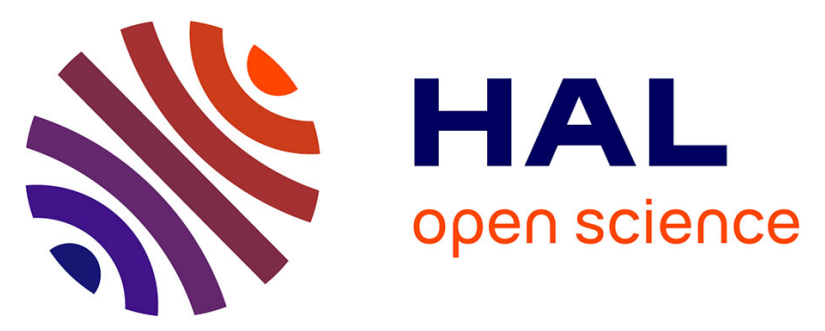

\title{
The issues of the uniqueness and the stability of the homogeneous response in uniaxial tests with gradient damage models
}

Kim Pham, Jean-Jacques Marigo, Corrado Maurini

\section{To cite this version:}

Kim Pham, Jean-Jacques Marigo, Corrado Maurini. The issues of the uniqueness and the stability of the homogeneous response in uniaxial tests with gradient damage models. Journal of the Mechanics and Physics of Solids, 2011, 59 (6), pp.1163-1190. 10.1016/j.jmps.2011.03.010 . hal-00578995

\section{HAL Id: hal-00578995 \\ https://hal.science/hal-00578995}

Submitted on 22 Mar 2011

HAL is a multi-disciplinary open access archive for the deposit and dissemination of scientific research documents, whether they are published or not. The documents may come from teaching and research institutions in France or abroad, or from public or private research centers.
L'archive ouverte pluridisciplinaire HAL, est destinée au dépôt et à la diffusion de documents scientifiques de niveau recherche, publiés ou non, émanant des établissements d'enseignement et de recherche français ou étrangers, des laboratoires publics ou privés. 


\title{
The issues of the uniqueness and the stability of the homogeneous response in uniaxial tests with gradient damage models
}

\author{
Kim Pham ${ }^{\mathrm{a}, \mathrm{b}}$, Jean-Jacques Marigo ${ }^{\mathrm{c}, *}$, Corrado Maurini ${ }^{\mathrm{a}, \mathrm{b}}$ \\ ${ }^{a}$ Université Paris 6 (UPMC), 4 place Jussieu, 75252 Paris, France \\ ${ }^{b}$ Institut Jean Le Rond d'Alembert (UMR-CNRS 7190), 4 place Jussieu, 75252 Paris, France \\ ${ }^{c}$ Laboratoire de Mécanique des Solides, Ecole Polytechnique, 91128 Palaiseau Cedex
}

\begin{abstract}
We consider a wide class of gradient damage models which are characterized by two constitutive functions after a normalization of the scalar damage parameter. The evolution problem is formulated following a variational approach based on the principles of irreversibility, stability and energy balance. Applied to a monotonically increasing traction test of a one-dimensional bar, we consider the homogeneous response where both the strain and the damage fields are uniform in space. In the case of a softening behavior, we show that the homogeneous state of the bar at a given time is stable provided that the length of the bar is less than a state dependent critical value and unstable otherwise. However, we show also that bifurcations can appear even if the homogeneous state is stable. All these results are obtained in a closed form. Finally, we propose a practical method to identify the two constitutive functions. This method is based on the measure of the homogeneous response in a situation where this response is stable without possibility of bifurcation, and on a procedure which gives the opportunity to detect its loss of stability. All the theoretical analysis is illustrated by examples.

Keywords: damage mechanics, gradient damage model, variational methods, irreversibility, stability, energy balance, bifurcation, Rayleigh ratio
\end{abstract}

\section{Introduction}

Which credit to grant to the global response of a uniaxial test in presence of softening? The generally admitted answer is: none! The advanced argument is that the experimental curve

${ }^{*}$ Corresponding author: marigo@lms.polytechnique.fr 
is not really representative of the intrinsic material behavior but a mixture of structural and material effects. The reason should be that the fields cannot be homogeneous in the sample because of the inevitable presence of localizations of the deformation in softening materials. For quasi-brittle materials, this empirical conclusion is reinforced by theoretical arguments based on local damage models, i.e. on models where the evolution of the damage at a point merely depends on the history of the strain at this point (Bažant, 1976). Indeed, whatever the method used, as well that based on the loss of ellipticity (Hill, 1958) as that based on the loss of stability (Nguyen, 2000; Marigo, 2000), the conclusion is the same: the homogeneous response is not observable. But a careful study (Marigo, 2000) shows that all the (infinite number of) responses predicted by such a local model are not observable. The reason is that a further localization of the deformation is always preferable from an energetic viewpoint.

Accordingly, if one assumes that in reality the localization is necessarily bounded from below and that a limit curve does exist, then one must change the model and introduce some characteristic length in order to penalize the too localized deformations. That leads to the concept of non local damage models which may be classified on the basis of the regularization technique. One can distinguish: (i) the integral procedure which makes use of a weight function and integrates the state variables over a typical domain whose size is related to the characteristic length (Pijaudier-Cabot and Bazant, 1987); (ii) the gradient scheme which introduces higher order terms with respect to spatial derivatives in the governing equations (Frémond and Nedjar, 1996; Comi and Perego, 2001; Pham and Marigo, 2010b). In both cases, the non-local terms may involve the displacement field, the damage variable, or both (Lorentz and Andrieux, 2003).

Once this non local approach was introduced, almost all theoretical studies, mainly developed in a one-dimensional context, have focussed on the role of the non-local terms for controlling the size of the localization zone. Some of them investigate the loss of ellipticity of the governing equations by meaning of a wave propagation analysis (Hill, 1962; Benallal et al., 1993; PijaudierCabot and Benallal, 1993). They show that the smallest wavelength that can propagate in an infinite softening homogeneous medium is proportional to the characteristic length. They assimilate this wavelength to the width of the localization zone in a generic boundary value problem for a structure with a finite dimension. Other works were devoted to the direct construction of the localization in a finite structure under specific boundary conditions, studying its evolution up to failure. Bažant and Zubelewicz (1988) and Benallal and Marigo (2007), for instance, have proposed, respectively in a non-local setting and a gradient damage context, a complete study of the localizations for particular damage laws, exhibiting explicit solutions for the localization profile. In both cases it was shown that the size of the localization is proportional to the internal length.

In the same time, the issue of the pertinence of the global response and the question whether the homogeneous states are observable became of secondary interest. Only few papers focus on these aspects and its relevance for the identification of the damage law (see e.g. Mazars et al. (1988); Bažant and Pijaudier-Cabot (1989)). Our paper is devoted to these two questions 
and, even more, we will use the properties of stability of the homogeneous states to identify the constitutive parameters and functions of the gradient damage model. The present work extends the previous (published or unpublished) results established in a more restrictive context (Marigo, 2000; Benallal and Marigo, 2007; Pham et al., 2010). Here, we make a precise effort to keep the discussion as general as possible, and to restrict to the minimum the arbitrariness of the assumptions on the constitutive laws. Considering a wide class of models, we classify them on the basis on the qualitative properties of their response in a onedimensional traction test. To this end, we perform a detailed analytical study of the homogenous response, including a complete discussion of the possible loss of stability and bifurcation toward localized solutions.

All the analysis relies on the very fecund variational approach which gives a sound theoretical framework for constructing and analyzing rate independent behaviors (Mielke, 2005; Bourdin et al., 2008; Pham and Marigo, 2010a,b). The basic ingredients of the variational approach are as follows: first, one defines the state variables of the model which can be of local or global nature; then, one defines the total energy of the system associated with its current state. This total energy includes the potential as well as the dissipated energy; finally, one formulates the evolution problem on the basis of three fundamental physical principles: (i) the irreversibility of some state variables (here the damage parameter), (ii) a stability condition and (iii) an energy balance. The irreversibility allows us to discriminate between the potential and the dissipated energy in the total energy, and to define the concept of accessible states; the stability condition requires that the current state realizes, at each time, a local minimum of the total energy among all accessible close states.; the energy balance takes a form which permits to handle time discontinuous evolutions. The origin of such an approach in damage mechanics can be found in the standard evolution law postulated by Marigo (1981) and then justified by Marigo (1989) by energetic arguments. The next step was to formulate the concepts of stability and bifurcation in terms of energetic variational principles (Nguyen, 1987, 2000). The last step which leads to the present formulation can be found in Pham and Marigo (2010a,b)) and is based on the work of Mielke (2005). A great amount of works devoted to fracture mechanics and based on this approach have been achieved in the last decade (see Bourdin et al., 2008, for a general survey). The main advantages of the variational approach are to give a unified setting for the analysis of existence, uniqueness and stability of quasi-static solutions and their numerical treatment (Bourdin, 2007; Amor et al., 2009). Indeed, while in the classical approaches the concept of stability is an extra assumption which can be seen as a post-treatment, in the variational approach the stability is one of the components of the evolution problem. In particular, what is generally considered as separate assumptions like the equilibrium equations, the boundary conditions and the laws governing the evolution of the internal variables become here a consequence of the three physical principles and of the choice of the energy functional. Thus, in the context of gradient damage models, the non local damage criterion and the natural damage boundary conditions are automatically deduced from the variational formulation. The "classical" Kuhn-Tucker conditions, see for example (Comi, 2001; Geers et al., 2000; Lorentz and Andrieux, 1999; Lorentz and Benallal, 2005; Peerlings et al., 2001), are derived from the first order stability condition and the energy balance, while the main results of stability and 
bifurcation come from the second order stability conditions and some properties of the second derivative of the total energy.

Specifically, the paper is structured as follows. Section 2 is devoted to the construction of the gradient damage model and to the setting of the evolution problem. First, we introduce two general families of gradient damage models, the so called strongly brittle materials and weakly brittle materials. After a normalization of the scalar damage parameter, each family is characterized by two functions of this parameter. Then, the evolution problem is formulated in terms of the three physical principles of irreversibility, stability and energy balance. Even if these principles have a more general scope, they are written here in a unidimensional setting only. In Section 3, we study the evolution problem of a bar submitted to a monotonically increasing traction at one end and we consider the particular solution where the strain and the damage fields are uniform in space. This particular evolution is called the homogeneous response. We discuss the properties of the resulting stress-strain curve and we analyze the stability of the homogeneous state associated with each value of the prescribed overall strain. That allows us to highlight size effects and to introduce the fundamental function giving the critical length of the bar beyond which the homogeneous state is no more stable. In Section 4, we study the possibility of bifurcation from the homogeneous branch by setting first the bifurcation problem. Then we show that a bifurcation to a stable branch is possible for a bar length smaller than the critical length associated to the loss of stability of the homogeneous state. Section 5 presents a possible method for the identification of the two state functions which characterize the behavior of the material. Since the homogeneous response is the unique possible response if the length of the bar is sufficiently small, we explain first how one function can be obtained from the associated stress-strain curve. Then, we propose a procedure for detecting the loss of stability of the homogeneous state which gives access to the second state function. Two (long) appendices are devoted to the solution of generic variational problems which are related to the stability and the uniqueness of the homogeneous response.

The following notations are used: the dependence on the time parameter $t$ is indicated by a subscript whereas the dependence on the spatial coordinate $x$ is indicated classically by parentheses, e.g. $x \mapsto u_{t}(x)$ stands for the displacement field at time $t$. In general, the functions of the damage parameter are represented by sans serif letters, like $\mathrm{E}(\alpha), \mathrm{S}(\alpha)$ or $\mathrm{L}(\alpha)$. The prime denotes either the derivative with respect to $x$ or the derivative with respect to the damage parameter, the dot stands for the time derivative, e.g. $u_{t}^{\prime}(x)=\partial u_{t}(x) / \partial x, \mathrm{E}^{\prime}(\alpha)=d \mathrm{E}(\alpha) / d \alpha$, $\dot{u}_{t}(x)=\partial u_{t}(x) / \partial t$. An intensive use of variational methods is made and most classical results are given without details. The reader not familiar with these tools is invited to refer to relevant textbooks, e.g. Dacorogna (1992). 


\section{Setting of the damage evolution problem}

\subsection{Construction of the gradient damage model}

Since we will only consider uniaxial tests, the construction of the model is simply made in a one-dimensional framework, but the procedure can easily be extended in a full three-dimensional setting, see Pham and Marigo (2010a,b). Accordingly, we consider a one-dimensional body made of an elastic damaging material whose behavior is defined as follows:

- The damage state of the body at point $x$ is characterized by a scalar parameter which is temporarily denoted by $D(x)$ (a change of variable will be made later so that the damage variable be finally denoted by $\alpha$ ). Without loss of generality, we can assume that $D(x)=0$ corresponds to the undamaged state of the point. The fully damaged state of the point, that is when the point cannot sustain any stress any more, corresponds to $D_{m}$, with $D_{m}$ possibly infinite.

- Let us denote by $\varepsilon(x)$ the strain at $x$ and by $D^{\prime}(x)=\frac{d D}{d x}(x)$ the gradient of damage at $x$. The triplet $\left(\varepsilon(x), D(x), D^{\prime}(x)\right)$ constitutes the state of point $x$.

- In order that the state of a point goes from the natural undamaged state $(0,0,0)$ to the state $\left(\varepsilon, D, D^{\prime}\right) \in \mathbb{R} \times\left[0, D_{m}\right] \times \mathbb{R}$, the exterior must supply an energy (by unit of length) $W\left(\varepsilon, D, D^{\prime}\right)$. This supplied energy is independent of the path followed to reach $\left(\varepsilon, D, D^{\prime}\right)$.

As we will see later, once the choice of the damage parameter is made, the knowledge of its maximal value $D_{m}$ and of the state function $\left(\varepsilon, D, D^{\prime}\right) \mapsto W\left(\varepsilon, D, D^{\prime}\right)$ is sufficient to characterize the behavior of the material. In particular the stress $\sigma(x)$ at point $x$ is given by

$$
\sigma(x)=\frac{\partial W}{\partial \varepsilon}\left(\varepsilon(x), D(x), D^{\prime}(x)\right) .
$$

In order to restrict the generality let us progressively introduce some additional assumptions on the state function $W$.

H1 The body is homogeneous. Hence, the constant $D_{m}$ and the state function $W$ do not depend explicitly on $x$;

H2 The material is isotropic. Hence, since the symmetry $x \mapsto-x$ changes $D^{\prime}$ into $-D^{\prime}, W$ must satisfy $W\left(\varepsilon, D,-D^{\prime}\right)=W\left(\varepsilon, D, D^{\prime}\right)$;

H3 No residual stress exists in any unstrained state. Hence $\frac{\partial W}{\partial \varepsilon}\left(0, D, D^{\prime}\right)=0$.

H4 The model is developed in the framework of small perturbations in terms of the strain and the gradient of damage. Accordingly, assuming that $\varepsilon$ and $D^{\prime}$ remain sufficiently close to $(0,0)$, the energy density function $W$ is expanded up to the second order with respect to $\varepsilon$ and $D^{\prime}$. Specifically, $W$ can read as

$$
W\left(\varepsilon, D, D^{\prime}\right)=w_{0}(D)+\sigma_{0}(D) \varepsilon+w_{1}(D) D^{\prime}+\frac{1}{2} w_{2}(D) D^{\prime 2}+\sigma_{1}(D) \varepsilon D^{\prime}+\frac{1}{2} E(D) \varepsilon^{2} .
$$


But $\mathbf{H 2}$ implies that $w_{1}(D)=\sigma_{1}(D)=0$ and $\mathbf{H 3}$ implies that $\sigma_{0}(D)=\sigma_{1}(D)=0$. Therefore, in the present context $W$ is reduced to

$$
W\left(\varepsilon, D, D^{\prime}\right)=w_{0}(D)+\frac{1}{2} w_{2}(D) D^{\prime 2}+\frac{1}{2} E(D) \varepsilon^{2} .
$$

The stress is then given by $\sigma=E(D) \varepsilon$ which allows us to interpret $E(D)$ as the Young modulus of the material in the damaged state $D$.

H5 The function $D \mapsto w_{0}(D)$ is continuously differentiable, strictly increasing and $w_{0}(0)=0$. (This assumption is natural since $w_{0}(D)$ represents the energy dissipated by the material during a damage process where $D^{\prime}$ is always equal to 0 , see Section 3.2.)

H6 The function $D \mapsto w_{2}(D)$ is positive. (This assumption is essential in order that the gradient damage term plays a regularizing role.)

Remark 1. We only consider uniaxial tests where the material is always either in tension or in compression. Therefore, it is not necessary to discriminate the behavior according to the sign of $\varepsilon$. But, of course, the constitutive functions $D \mapsto E(D), D \mapsto w_{0}(D)$ and even $D \mapsto w_{2}(D)$ are (in general) different in tension and in compression. We could also make the damage variable dependent on the sign of $\varepsilon$ like in Mazars (1986). In a general way, a careful modeling of the asymmetric behavior in tension and compression is outside the scope of the present paper and is reserved for future works.

At this stage of the construction, the damage parameter $D$ has a purely phenomenological role. It is quite licit to change the parameter without modifying the intrinsic behavior of the material. Accordingly, it is possible to reduce the number of constitutive functions to be determined from three to two with the help of a change of the damage variable. Many choices are possible, cf Pham and Marigo (2010a,b). For example, a way consists in transforming the function $D \mapsto w_{2}(D)$ into a constant. We make here another choice which consists in choosing the "homogeneously" dissipated energy as the damage variable (except for a multiplicative factor). This choice makes easier the interpretation and the identification of the constitutive functions. Specifically, one proceeds as follows.

First, since $D \mapsto w_{0}(D)$ is strictly increasing by virtue of $\mathbf{H 5}$, we can consider its inverse function. Then we distinguish two cases according to whether $w_{0}\left(D_{m}\right)$ is finite or infinite.

Case $w_{0}\left(D_{m}\right)<+\infty$. Let us set $\mathrm{w}_{1}=w_{0}\left(D_{m}\right)$ and choose as the new damage variable $\alpha=w_{0}(D) / \mathrm{w}_{1}$. Then, $\alpha$ grows from 0 to 1 when $D$ grows from 0 to $D_{m}$ and the second order term $w_{2}(D) D^{\prime 2}$ becomes

$$
w_{2}(D) D^{\prime 2}=w_{2}\left(D^{-1}(\alpha)\right) \frac{\mathrm{w}_{1}^{2} \alpha^{\prime 2}}{w_{0}^{\prime}\left(D^{-1}(\alpha)\right)^{2}} .
$$

Since $w_{2}>0$ by virtue of $\mathbf{H 6}$, the coefficient of $\alpha^{\prime 2}$ is also positive and can read as $\mathrm{w}_{1} \mathrm{~L}(\alpha)^{2}$ where $\mathrm{L}(\alpha)>0$ has the dimension of a length. Accordingly, the energy density can finally 
read as the function $\mathbf{W}$ defined on $\mathbb{R} \times[0,1] \times \mathbb{R}$ by

$$
\mathrm{W}\left(\varepsilon, \alpha, \alpha^{\prime}\right)=\mathrm{w}_{1} \alpha+\frac{1}{2} \mathrm{~W}_{1} \mathrm{~L}(\alpha)^{2} \alpha^{\prime 2}+\frac{1}{2} \mathrm{E}(\alpha) \varepsilon^{2}
$$

where $\mathrm{E}(\alpha)$ denotes now the function giving the Young modulus in terms of $\alpha$. This type of model will be called strongly brittle material.

Case $w_{0}\left(D_{m}\right)=+\infty$. Then we choose arbitrarily $D_{1} \in\left(0, D_{m}\right)$, set $\mathrm{w}_{1}=w_{0}\left(D_{1}\right)$ and take as the new damage variable $\alpha=w_{0}(D) / \mathrm{w}_{1}$. Then, $\alpha$ grows from 0 to $\infty$ when $D$ grows from 0 to $D_{m}$. The second order term $w_{2}(D) D^{\prime 2}$ can be written $\mathrm{w}_{1} \mathrm{~L}(\alpha)^{2} \alpha^{\prime 2}$ again. Finally, the energy density $W$ can still read as in (2) but is now defined on $\mathbb{R} \times[0,+\infty) \times \mathbb{R}$. This type of model will be called weakly brittle material.

Thus, in both cases the energy density takes the same form, the range of damage parameter only differs from one case to the other. Accordingly, in order to determine the behavior of the material, one must identify the two functions $\mathrm{L}(\alpha)$ and $\mathrm{E}(\alpha)$, the constant $\mathrm{w}_{1}$ and whether $\alpha_{m}:=\sup \alpha=1$ or $\infty$.

The qualitative properties of the damage model, in particular its softening or hardening character, strongly depend on some properties of the stiffness function $\alpha \mapsto \mathrm{E}(\alpha)$, the compliance function $\alpha \mapsto \mathrm{S}(\alpha)=1 / \mathrm{E}(\alpha)$ and their derivatives. From now on, we adopt the following hypotheses:

Hypothesis 1 (Constitutive assumptions). For given $\alpha_{m} \in\{1,+\infty\}$, the functions $\alpha \mapsto \mathrm{E}(\alpha)$, $\alpha \mapsto \mathrm{S}(\alpha)$ and $\alpha \mapsto \mathrm{L}(\alpha)$ enjoy the following properties on their domain of definition $\mathbb{D}=$ $\left[0, \alpha_{m}\right)$ :

(i) The stiffness function $\alpha \mapsto \mathrm{E}(\alpha)$ is positive, decreasing from $\mathrm{E}_{0}$ to 0 and twice continuously differentiable. Its derivative $\alpha \mapsto \mathrm{E}^{\prime}(\alpha)$ is negative, increasing from $-\mathrm{E}_{0}^{\prime}$ to 0 :

$$
\begin{gathered}
\mathrm{E}(0)=\mathrm{E}_{0}>0, \quad \mathrm{E}\left(\alpha_{m}\right)=0, \quad \mathrm{E}^{\prime}\left(\alpha_{m}\right)=0 \\
\forall \alpha \in\left[0, \alpha_{m}\right), \quad \mathrm{E}(\alpha)>0, \quad \mathrm{E}^{\prime}(\alpha)<0, \quad \mathrm{E}^{\prime \prime}(\alpha)>0 .
\end{gathered}
$$

(ii) Therefore, the compliance function $\alpha \mapsto \mathrm{S}(\alpha)$ is positive, increasing from $\mathrm{S}_{0}=1 / \mathrm{E}_{0}$ to $\infty$ and twice continuously differentiable:

$$
\mathrm{S}(0)=1 / \mathrm{E}_{0}, \quad \mathrm{~S}\left(\alpha_{m}\right)=+\infty ; \quad \forall \alpha \in\left[0, \alpha_{m}\right), \quad \mathrm{S}(\alpha)>0, \quad \mathrm{~S}^{\prime}(\alpha)>0 .
$$

Moreover, according to the sign of the second derivative $\mathrm{S}^{\prime \prime}$, the behavior is either softening or hardening, see Section 3. Specifically, we will distinguish

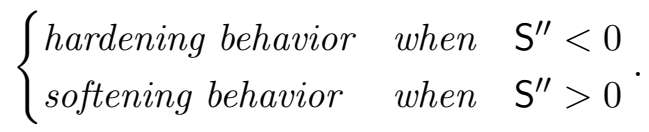

As we will show in Section 3.1 (see cases 1 and 2 page 14), these definitions of hardening and softening behaviors lead to the same concepts of hardening and softening as those which are usually introduced in terms of the monotonicity of the stress-strain response. 
(iii) $\alpha \mapsto \mathrm{L}(\alpha)$ is positive and twice continuously differentiable.

Let us illustrate the different types of models with the two following families:

Example 1. A family of models which satisfies the assumptions above with $\alpha_{m}=1$ and then corresponds to strongly brittle materials is the following one when $q>1$ :

$$
\mathrm{E}(\alpha)=\mathrm{E}_{0}(1-\alpha)^{q}, \quad \mathrm{w}_{1}=\frac{q \sigma_{\mathrm{c}}^{2}}{2 \mathrm{E}_{0}}, \quad \mathrm{~L}(\alpha)=(1-\alpha)^{p} \ell_{\mathrm{c}} .
$$

It contains five material parameters: the sound Young modulus $\mathrm{E}_{0}>0$, the dimensionless parameters $p \in \mathbb{R}$ and $q>1$, the critical stress $\sigma_{\mathrm{c}}>0$ and the internal length $\ell_{\mathrm{c}}>0$ (see their interpretation in Section 3). Moreover, since $\mathrm{S}^{\prime \prime}(\alpha)=q(q+1)(1-\alpha)^{-q-2} \mathrm{~S}_{0}>0$, all these models lead to a softening behavior.

Example 2. Another family of models which satisfies the assumptions above with $\alpha_{m}=\infty$ and then corresponds to weakly brittle materials is the following one when $q>0$ :

$$
\mathrm{E}(\alpha)=\frac{\mathrm{E}_{0}}{(1+\alpha)^{q}}, \quad \mathrm{w}_{1}=\frac{q \sigma_{\mathrm{c}}^{2}}{2 \mathrm{E}_{0}}, \quad \mathrm{~L}(\alpha)=\frac{\ell_{\mathrm{c}}}{(1+\alpha)^{p}} .
$$

It also contains five material parameters: the sound Young modulus $\mathrm{E}_{0}>0$, the dimensionless parameters $p \in \mathbb{R}$ and $q>0$, the critical stress $\sigma_{\mathrm{c}}>0$ and the internal length $\ell_{\mathrm{c}}>0$ (see their interpretation in Section 3). Moreover, since $\mathrm{S}^{\prime \prime}(\alpha)=q(q-1)(1+\alpha)^{q-2} \mathrm{~S}_{0}$, the models with $0<q<1$ lead to a hardening behavior while those with $q>1$ to a softening behavior.

\subsection{The evolution problem}

\subsubsection{Formulation}

Let $(0, L)$ be the natural reference configuration of a homogeneous one-dimensional bar. The bar is made of the damaging material characterized by the energy density function $\mathrm{W}$ given by (2). The end $x=0$ of the bar is fixed, whereas the displacement of the end $x=L$ is prescribed to a value $U_{t}$ depending on an increasing parameter $t \geq 0$ which plays the role of the "time":

$$
u_{t}(0)=0, \quad u_{t}(L)=U_{t}, \quad t \geq 0 .
$$

All the analysis is made in a quasi-static setting. The equilibrium state of the bar at time $t$ is characterized by the pair $\left(u_{t}, \alpha_{t}\right)$ of the displacement and damage fields. Assuming that the bar is undamaged at $t=0$, the evolution problem consists in finding $t \mapsto\left(u_{t}, \alpha_{t}\right)$ for $t \in\left[0, t_{r}\right)$ where $t_{r}$ denotes the time at which the bar breaks, i.e. when the damage field takes the critical value $\alpha_{m}$ somewhere in the interior or at the ends of the bar:

$$
t_{r}=\sup \left\{t \geq 0: \sup _{x \in[0, L]} \alpha_{t}(x)<\alpha_{m}\right\} .
$$


The time $t_{r}$ is not known in advance and must be determined. The evolution of the displacement and the damage in the bar is obtained via a variational formulation, the main ingredients of which are recalled hereafter, see Pham and Marigo (2010a,b) for details.

I. First, we associate with any pair $(u, \alpha)$ of displacement and damage fields the total energy $\mathcal{E}(u, \alpha)$ of the bar

$$
\mathcal{E}(u, \alpha)=\int_{0}^{L}\left(\frac{1}{2} \mathrm{w}_{1} \mathrm{~L}(\alpha(x))^{2} \alpha^{\prime}(x)^{2}+\frac{1}{2} \mathrm{E}(\alpha(x)) u^{\prime}(x)^{2}+\mathrm{w}_{1} \alpha(x)\right) d x
$$

where the prime denotes the derivative with respect to $x$.

II. Then, we define the set of admissible fields. If we consider only damage fields without failure, i.e. $\alpha$ such that $\sup _{x \in[0, L]} \alpha(x)<\alpha_{m}$, then the energy is finite provided that both $u$ and $\alpha$ belong to $H^{1}(0, L)$, the Sobolev space of functions which are square integrable and the weak derivative of which is also square integrable (Brezis, 1983). Accordingly, the affine space of kinematically admissible displacement fields at time $t$, say $\mathcal{C}_{t}$, and its associated linear space $\mathcal{C}^{0}$ are given by

$$
\begin{aligned}
\mathcal{C}_{t} & =\left\{v \in H^{1}(0, L): v(0)=0, v(L)=U_{t}\right\}, \\
\mathcal{C}^{0} & =H_{0}^{1}(0, L)=\left\{v \in H^{1}(0, L): v(0)=v(L)=0\right\} .
\end{aligned}
$$

The convex set of admissible damage fields without failure $\mathcal{D}$ and the convex cone of admissible damage evolution fields $\mathcal{D}_{+}$are defined by

$$
\mathcal{D}=\left\{\alpha \in H^{1}(0, L): \alpha \geq 0, \sup _{[0, L]} \alpha<\alpha_{m}\right\}, \quad \mathcal{D}_{+}=\left\{\beta \in H^{1}(0, L): \beta \geq 0\right\} .
$$

Let us note that for each $\alpha$ in $\mathcal{D}, \alpha$ is continuous on $[0, L]$ and hence $\max _{x \in[0, L]} \alpha(x)<\alpha_{m}$.

Remark 2. We do not introduce any constraint on the damage at the ends of the bar. The consequence will be that natural boundary conditions will appear by virtue of the stability and energy balance principles. This absence of constraint is essential for obtaining homogeneous evolutions.

III. Finally, the evolution problem consists in finding $t_{r}$ and, for $t \in\left[0, t_{r}\right)$, the displacement and damage fields $\left(u_{t}, \alpha_{t}\right) \in \mathcal{C}_{t} \times \mathcal{D}$ which have to satisfy the three following items

(IR) Irreversibility of the damage evolution: the damage $t \mapsto \alpha_{t}$ starts from 0 at $t=0$ and must be a non decreasing function of $t$;

(ST) Stability of the state with respect to perturbations in the direction of accessible states: at every $t$, the state $\left(u_{t}, \alpha_{t}\right)$ must be directionally stable in the following sense

$$
\begin{gathered}
\forall(v, \beta) \in \mathcal{C}^{0} \times \mathcal{D}_{+}, \quad \exists \bar{h}>0: \quad \forall h \in[0, \bar{h}], \\
\left(u_{t}+h v, \alpha_{t}+h \beta\right) \in \mathcal{C}_{t} \times \mathcal{D}, \quad \mathcal{E}\left(u_{t}+h v, \alpha_{t}+h \beta\right) \geq \mathcal{E}\left(u_{t}, \alpha_{t}\right) .
\end{gathered}
$$

That means that, to perturb the bar in any way compatible with the boundary conditions and the irreversibility of damage, the exterior must supply an amount of energy (at least for sufficiently small perturbations); 
(EB) Energy balance during the evolution: at every $t$, the total energy $\mathcal{E}\left(u_{t}, \alpha_{t}\right)$ must satisfy the following balance of energy:

$$
\mathcal{E}\left(u_{t}, \alpha_{t}\right)-\mathcal{E}\left(u_{0}, \alpha_{0}\right)=\int_{0}^{t} \sigma_{\tau}(L) \dot{U}_{\tau} d \tau
$$

where $\sigma_{\tau}$ denotes the stress field at time $\tau$, i.e.

$$
\sigma_{\tau}(x)=\mathrm{E}\left(\alpha_{\tau}(x)\right) u_{\tau}^{\prime}(x)
$$

In the present context, the energy balance simply requires that the variation of the total energy be equal to the work done by the external force. The interested reader must refer to Pham and Marigo (2010a,b) for a general discussion on the formulation of the energy balance in the context of damage mechanics, to Mielke (2005) for general rate independent behaviors and to Bourdin et al. (2008) for its use in the variational approach to fracture.

\subsubsection{The first order stability condition}

A necessary condition for the stability of a state in the sense (ST) is the non-negativeness of the first variation of the total energy for the admissible evolutions of the state variables. We will refer to this first-order stability condition as (st). Its consequences are illustrated in the following proposition.

Proposition 2.1. Let $\left(u_{t}, \alpha_{t}\right)$ be a solution of the evolution problem. Then, at each time, the so-called first order stability condition (st) is satisfied

$$
\forall(v, \beta) \in \mathcal{C}^{0} \times \mathcal{D}_{+}, \quad \mathcal{E}^{\prime}\left(u_{t}, \alpha_{t}\right)(v, \beta) \geq 0
$$

where $\mathcal{E}^{\prime}$ denotes the directional derivative of $\mathcal{E}$, i.e.

$$
\mathcal{E}^{\prime}(u, \alpha)(v, \beta)=\int_{0}^{L}\left(\mathrm{E}(\alpha) u^{\prime} v^{\prime}+\left(\frac{1}{2} \mathrm{E}^{\prime}(\alpha) u^{\prime 2}+\mathrm{w}_{1}+\mathrm{w}_{1} \mathrm{~L}(\alpha) \mathrm{L}^{\prime}(\alpha) \alpha^{\prime 2}\right) \beta+\mathrm{w}_{1} \mathrm{~L}(\alpha)^{2} \alpha^{\prime} \beta^{\prime}\right) d x .
$$

In turn, (st) is equivalent to the two following items

(i) Mechanical equilibrium: The stress $\sigma_{t}$ is constant along the bar and related to the damage field by

$$
\sigma_{t}=\frac{U_{t}}{\int_{0}^{L} \mathrm{~S}\left(\alpha_{t}(x)\right) d x} .
$$

(ii) Non local damage criterion : The damage field $\alpha_{t}$ satisfies the weak form of the damage criterion

$$
\int_{0}^{L} \mathrm{w}_{1} \mathrm{~L}\left(\alpha_{t}\right)^{2} \alpha_{t}^{\prime} \beta^{\prime} d x+\int_{0}^{L}\left(-\frac{1}{2} \mathrm{~S}^{\prime}\left(\alpha_{t}\right) \sigma_{t}^{2}+\mathrm{w}_{1}+\mathrm{w}_{1} \mathrm{~L}\left(\alpha_{t}\right) \mathrm{L}^{\prime}\left(\alpha_{t}\right) \alpha_{t}^{\prime 2}\right) \beta d x \geq 0, \quad \forall \beta \in \mathcal{D}_{+}
$$


which can take the following strong form when $\alpha_{t}$ belongs to $H^{2}(0, L)$ :

$$
\left\{\begin{array}{l}
\frac{\partial}{\partial x}\left(\mathrm{w}_{1} \mathrm{~L}\left(\alpha_{t}\right)^{2} \frac{\partial \alpha_{t}}{\partial x}\right)+\frac{1}{2} \mathrm{~S}^{\prime}\left(\alpha_{t}\right) \sigma_{t}^{2} \leq \mathrm{w}_{1}+\mathrm{w}_{1} \mathrm{~L}\left(\alpha_{t}\right) \mathrm{L}^{\prime}\left(\alpha_{t}\right) \alpha_{t}^{\prime 2} \quad \text { a. e. in }(0, L), \\
\alpha_{t}^{\prime}(0) \leq 0, \quad \alpha_{t}^{\prime}(L) \geq 0 .
\end{array}\right.
$$

Proof. Dividing (ST) by $h$ and passing to the limit when $h$ goes to 0 lead to (st). Choosing $\beta=0$ in (st), we obtain the weak form of the equilibrium of the bar

$$
\int_{0}^{L} \sigma_{t}(x) v^{\prime}(x) d x=0, \quad \forall v \in \mathcal{C}^{0}
$$

from which we immediately deduce that the stress is constant along the bar and hence

$$
\sigma_{t}=\mathrm{E}\left(\alpha_{t}(x)\right) u_{t}^{\prime}(x), \quad \forall x \in(0, L)
$$

Dividing (17) by $\mathrm{E}\left(\alpha_{t}(x)\right)$, integrating over $(0, L)$ and using the boundary conditions (5), we get (13). Inserting (13) into (st) leads to (14). This latter inequality can be read in the sense of distribution

$$
\frac{\partial}{\partial x}\left(\mathrm{w}_{1} \mathrm{~L}\left(\alpha_{t}\right)^{2} \frac{\partial \alpha_{t}}{\partial x}\right)+\frac{1}{2} \mathrm{~S}^{\prime}\left(\alpha_{t}\right) \sigma_{t}^{2} \leq \mathrm{w}_{1}+\mathrm{w}_{1} \mathrm{~L}\left(\alpha_{t}\right) \mathrm{L}^{\prime}\left(\alpha_{t}\right) \alpha_{t}^{\prime 2}
$$

When $\alpha_{t}$ is smooth enough, say in $H^{2}(0, L)$, then the inequality reads in the classical sense almost everywhere. Moreover, in such a case, $\alpha_{t}^{\prime}$ is continuous over $[0, L]$.

Taking $h>0$ small enough, $\beta(x)=(1-x / h)^{+}$where $a^{+}=\max \{a, 0\}$ and inserting into (14) gives

$$
-\frac{1}{h} \int_{0}^{h} \mathrm{w}_{1} \mathrm{~L}\left(\alpha_{t}\right)^{2} \alpha_{t}^{\prime} d x+\int_{0}^{h}\left(\mathrm{w}_{1}+\mathrm{w}_{1} \mathrm{~L}\left(\alpha_{t}\right) \mathrm{L}^{\prime}\left(\alpha_{t}\right) \alpha_{t}^{\prime 2}-\frac{1}{2} \mathrm{~S}^{\prime}\left(\alpha_{t}\right) \sigma_{t}^{2}\right)\left(1-\frac{x}{h}\right) d x \geq 0 .
$$

Passing to the limit when $h$ goes to 0 , we get $\alpha_{t}^{\prime}(0) \leq 0$. By the same procedure we get also $\alpha_{t}^{\prime}(L) \geq 0$

Conversely, it is easy to check that if $\alpha_{t} \in H^{2}(0, L)$ and (15) hold then (14) is satisfied and hence (st) holds.

\subsubsection{The energy balance for smooth evolutions}

Let us suppose that the fields evolve smoothly in time in such a way that the rates $\dot{u}_{t}$ and $\dot{\alpha}_{t}$ belong to $H^{1}(0, L)$. Differentiating (EB) with respect to $t$ leads to $0=\mathcal{E}^{\prime}\left(u_{t}, \alpha_{t}\right)\left(\dot{u}_{t}, \dot{\alpha}_{t}\right)-\sigma_{t} \dot{U}_{t}$. 
Since $\dot{u}(0)=0$ and $\dot{u}(L)=\dot{U}_{t}$, we have $\int_{0}^{L} \mathrm{E}\left(\alpha_{t}\right) u_{t}^{\prime} \dot{u}_{t}^{\prime} d x=\sigma_{t} \dot{U}_{t}$ and we finally obtain the new form of the energy balance:

$$
0=\int_{0}^{L}\left(\left(-\frac{1}{2} \mathrm{~S}^{\prime}\left(\alpha_{t}\right) \sigma_{t}^{2}+\mathrm{w}_{1}+\mathrm{w}_{1} \mathrm{~L}\left(\alpha_{t}\right) \mathrm{L}^{\prime}\left(\alpha_{t}\right) \alpha_{t}^{\prime 2}\right) \dot{\alpha}_{t}+\mathrm{w}_{1} \mathrm{~L}\left(\alpha_{t}\right)^{2} \alpha_{t}^{\prime} \dot{\alpha}_{t}^{\prime}\right) d x .
$$

When the solution is also smooth in space, after an integration by parts the equality above becomes

$$
\begin{aligned}
0= & \int_{0}^{L}\left(-\frac{1}{2} \mathrm{~S}^{\prime}\left(\alpha_{t}\right) \sigma_{t}^{2}+\mathrm{w}_{1}+\mathrm{w}_{1} \mathrm{~L}\left(\alpha_{t}\right) \mathrm{L}^{\prime}\left(\alpha_{t}\right) \alpha_{t}^{\prime 2}-\frac{\partial}{\partial x}\left(\mathrm{w}_{1} \mathrm{~L}\left(\alpha_{t}\right)^{2} \frac{\partial \alpha_{t}}{\partial x}\right)\right) \dot{\alpha}_{t} d x \\
& +\mathrm{w}_{1} \mathrm{~L}\left(\alpha_{t}(L)\right)^{2} \alpha_{t}^{\prime}(L) \dot{\alpha}_{t}(L)-\mathrm{w}_{1} \mathrm{~L}\left(\alpha_{t}(0)\right)^{2} \alpha_{t}^{\prime}(0) \dot{\alpha}_{t}(0) .
\end{aligned}
$$

Using the strong non local damage criterion (15) and the irreversibility condition $\dot{\alpha}_{t} \geq 0$, we finally obtain the conditions of coherency:

$$
\left\{\begin{array}{l}
\dot{\alpha}_{t}\left(-\frac{1}{2} \mathrm{~S}^{\prime}\left(\alpha_{t}\right) \sigma_{t}^{2}+\mathrm{w}_{1}+\mathrm{w}_{1} \mathrm{~L}\left(\alpha_{t}\right) \mathrm{L}^{\prime}\left(\alpha_{t}\right) \alpha_{t}^{\prime 2}-\frac{\partial}{\partial x}\left(\mathrm{w}_{1} \mathrm{~L}\left(\alpha_{t}\right)^{2} \frac{\partial \alpha_{t}}{\partial x}\right)\right)=0 \quad \text { a. e. in }(0, L) \\
\dot{\alpha}_{t}(0) \alpha_{t}^{\prime}(0)=0, \quad \dot{\alpha}_{t}(L) \alpha_{t}^{\prime}(L)=0
\end{array}\right.
$$

Remark 3. The set of equations (15)-(18) make sense and hence can be used only when the evolution is smooth both in space and time. Unfortunately, discontinuous in time evolutions due to snap-back phenomena are common in softening materials. In such a case, the energy balance principle (EB) is still meaningful and it implies that the total energy of the body is a absolutely continuous function of time even if the damage evolution is not continuous.

When $\mathrm{L}(\alpha)$ is a constant independent of $\alpha$, then (15)-(18) are the "classical" equations found in the literature, see Comi (1999) or Lorentz and Andrieux (2003). Let us note however that our variational approach gives a rational, unambiguous, systematic method for constructing in a general way the full set of equations including the often debated damage boundary conditions.

\section{The spatially homogeneous evolution and the issue of its stability}

We assume in this section and the next one that the prescribed displacement at the end $x=L$ of the bar is monotonically increasing and we set

$$
U_{t}=t L, \quad t \geq 0
$$

so that the dimensionless parameter $t$ represents the overall stretching of the bar. 


\subsection{The spatially homogeneous evolution}

A natural candidate to be a solution of the evolution problem is the response where both the damage and the strain fields are constant in space and evolve smoothly in time. Indeed, it is easy to check that, under Hypothesis 1, there exits a (unique) continuous in time and uniform in space evolution which satisfies the irreversibility condition, the first order stability condition and the balance of energy. Specifically, we have

Proposition 3.1. By virtue of the hypothesis that $\mathrm{E}^{\prime}<0$ and $\mathrm{E}^{\prime \prime}>0$ on $\mathbb{D}$ (see Hypothesis 1), the evolution $\left(u_{t}^{*}, \alpha_{t}^{*}\right)$ given by

$$
u_{t}^{*}=t x, \quad \alpha_{t}^{*}=\left\{\begin{array}{ll}
0 & \text { if } t<\varepsilon_{\mathrm{c}} \\
\left(-\frac{\mathrm{w}_{1}}{\mathrm{E}^{\prime}}\right)^{-1}\left(t^{2} / 2\right) & \text { if } t \geq \varepsilon_{\mathrm{c}}
\end{array} \quad \text { with } \quad \varepsilon_{\mathrm{c}}=\sqrt{\frac{2 \mathrm{w}_{1}}{-\mathrm{E}^{\prime}(0)}}\right.
$$

is the unique one such that the damage is uniform in space, absolutely continuous in time and satisfies (IR), (st) and (EB). In (20), $\left(-\mathrm{w}_{1} / \mathrm{E}^{\prime}\right)^{-1}\left(t^{2} / 2\right)$ denotes the value of the inverse function of $\alpha \mapsto-\mathrm{w}_{1} / \mathrm{E}^{\prime}(\alpha)$ at $t^{2} / 2$.

Proof. Let us first check that $\left(u_{t}^{*}, \alpha_{t}^{*}\right)$ given in the statement is continuously differentiable in time and satisfies (IR), (st) and (EB). By Hypothesis $1, \mathrm{E}^{\prime}<0, \mathrm{E}^{\prime \prime}>0$ and hence $\alpha \mapsto-\mathrm{w}_{1} / \mathrm{E}^{\prime}(\alpha)$ is a positive, continuously differentiable increasing function, growing from $\varepsilon_{\mathrm{c}}{ }^{2} / 2$ to $+\infty$ when $\alpha$ grows from 0 to $\alpha_{m}$. Therefore, $t \mapsto \alpha_{t}^{*}$ is continuous everywhere, continuously differentiable at every $t \neq \varepsilon_{c}$, monotonically increasing and hence satisfies (IR). Moreover, $\alpha_{t}^{*}$ satisfies

$$
\left\{\begin{array}{ll}
\frac{1}{2} \mathrm{E}^{\prime}\left(\alpha_{t}^{*}\right) t^{2}+\mathrm{w}_{1}>0 & \text { if } t<\varepsilon_{\mathrm{c}} \\
\frac{1}{2} \mathrm{E}^{\prime}\left(\alpha_{t}^{*}\right) t^{2}+\mathrm{w}_{1}=0 & \text { if } t \geq \varepsilon_{\mathrm{c}}
\end{array} .\right.
$$

Then, setting $\sigma_{t}^{*}=\mathrm{E}\left(\alpha_{t}^{*}\right) t$, we have at every $t \geq 0$

$$
\left(\sigma_{t}^{*}\right)^{2} \mathrm{~S}^{\prime}\left(\alpha_{t}^{*}\right)-2 \mathrm{w}_{1}=-\mathrm{E}^{\prime}\left(\alpha_{t}^{*}\right) t^{2}-2 \mathrm{w}_{1} \leq 0
$$

and hence (st) is satisfied, see (13)-(14). Finally, by construction, we have

$$
\mathcal{E}\left(u_{t}^{*}, \alpha_{t}^{*}\right)=\frac{1}{2} \mathrm{E}\left(\alpha_{t}^{*}\right) t^{2} L+\mathrm{w}_{1} \alpha_{t}^{*} L, \quad \mathcal{E}\left(u_{0}^{*}, \alpha_{0}^{*}\right)=0, \quad \int_{0}^{t} \sigma_{\tau} \dot{U}_{\tau} d \tau=L \int_{0}^{t} \mathrm{E}\left(\alpha_{\tau}^{*}\right) \tau d \tau
$$

and (EB) is satisfied when $t \leq \varepsilon_{\mathrm{c}}$. When $t>\varepsilon_{\mathrm{c}}$, we have $\mathrm{E}^{\prime}\left(\alpha_{t}^{*}\right) t^{2}+2 \mathrm{w}_{1}=0$ and hence

$$
\frac{d}{d t} \mathcal{E}\left(u_{t}^{*}, \alpha_{t}^{*}\right)=\mathrm{E}\left(\alpha_{t}^{*}\right) t L+\left(\frac{1}{2} \mathrm{E}^{\prime}\left(\alpha_{t}^{*}\right) t^{2}+\mathrm{w}_{1}\right) L \dot{\alpha}_{t}^{*}=\sigma_{t}^{*} L
$$

Integrating in time, we obtain (EB). Therefore $\left(u_{t}^{*}, \alpha_{t}^{*}\right)$ satisfies all the requirements. 
Let us now prove that $\left(u_{t}^{*}, \alpha_{t}^{*}\right)$ is the unique smooth uniform evolution which satisfies (IR), (st) and (EB). If $\alpha_{t}$ is uniform in space, then by the equilibrium equation (13) the strain is also uniform in space and therefore $u_{t}=t x=u_{t}^{*}$. Inserting this relation into (15) and (EB) leads to

$$
-\frac{1}{2} \mathrm{E}^{\prime}\left(\alpha_{t}\right) t^{2} \leq \mathrm{w}_{1}, \quad \frac{1}{2} \mathrm{E}\left(\alpha_{t}\right) t^{2}+\mathrm{w}_{1} \alpha_{t}=\int_{0}^{t} \mathrm{E}\left(\alpha_{\tau}\right) \tau d \tau
$$

By (IR), $t \mapsto \alpha_{t}$ is monotonic and hence differentiable almost everywhere. Differentiating the equality in (21) with respect to $t$, we obtain for almost all $t$

$$
\left(\frac{1}{2} \mathrm{E}^{\prime}\left(\alpha_{t}\right) t^{2}+\mathrm{w}_{1}\right) \dot{\alpha}_{t}=0 .
$$

Since $\alpha_{0}=0$, we deduce from (22) and the inequality in (21) that $\alpha_{t}=\alpha_{t}^{*}=0$ as long as $t^{2} \mathrm{E}_{0}^{\prime}+2 \mathrm{w}_{1}>0$, i.e. as long as $t<\varepsilon_{\mathrm{c}}$. By continuity, $\alpha_{t}=0$ and $t^{2} \mathrm{E}_{0}^{\prime}+2 \mathrm{w}_{1}=0$ at $t=\varepsilon_{\mathrm{c}}$. Let us prove that $t^{2} \mathrm{E}^{\prime}\left(\alpha_{t}\right)+2 \mathrm{w}_{1}=0$ when $t \geq \varepsilon_{\mathrm{c}}$. It is true at $t=\varepsilon_{\mathrm{c}}$. If it were not true at some $\tau>\varepsilon_{\mathrm{c}}$, then $\tau^{2} \mathrm{E}^{\prime}\left(\alpha_{\tau}\right)+2 \mathrm{w}_{1}>0$. By continuity, this inequality should hold in an interval $(s, \tau)$. Taking the infimum of such a $s$, we should have $s^{2} \mathrm{E}^{\prime}\left(\alpha_{s}\right)+2 \mathrm{w}_{1}=0$ (because the equality holds at $\varepsilon_{\mathrm{c}}$ and $\left.s \geq \varepsilon_{\mathrm{c}}\right)$. But almost everywhere in the interval $(s, \tau)$ we must also have $\dot{\alpha}_{t}=0$ by (22) and hence (because of the assumed absolute continuity of $\left.t \mapsto \alpha_{t}\right) \alpha_{s}=\alpha_{\tau}$. Therefore, we should have

$$
0=s^{2} \mathrm{E}^{\prime}\left(\alpha_{s}\right)+2 \mathrm{w}_{1}<\tau^{2} \mathrm{E}^{\prime}\left(\alpha_{\tau}\right)+2 \mathrm{w}_{1}=\tau^{2} \mathrm{E}^{\prime}\left(\alpha_{s}\right)+2 \mathrm{w}_{1}=\left(\tau^{2}-s^{2}\right) \mathrm{E}^{\prime}\left(\alpha_{s}\right)
$$

but it is impossible because $\tau>s$ and $\mathrm{E}^{\prime}\left(\alpha_{s}\right)<0$. Hence $t^{2} \mathrm{E}^{\prime}\left(\alpha_{t}\right)+2 \mathrm{w}_{1}=0$ when $t \geq \varepsilon_{\mathrm{c}}$ and so $\alpha_{t}=\alpha_{t}^{*}$. That proves the uniqueness.

Let us set

$$
\sigma_{\mathrm{c}}:=\sqrt{\frac{2 \mathrm{w}_{1}}{\mathrm{~S}^{\prime}(0)}}=\mathrm{E}_{0} \varepsilon_{\mathrm{c}},
$$

$\sigma_{\mathrm{c}}$ corresponds to the yield stress. During the damaging phase $\left(t \geq \varepsilon_{\mathrm{c}}\right)$, the stress $\sigma_{t}^{*}$ satisfies

$$
\frac{1}{2} \mathrm{~S}^{\prime}\left(\alpha_{t}^{*}\right) \sigma_{t}^{* 2}=\mathrm{w}_{1}
$$

and is given by

$$
\sigma_{t}^{*}=\mathrm{F}(t) \quad \text { with } \quad \mathrm{F}(t)=t \mathrm{E}\left(\left(-\frac{\mathrm{w}_{1}}{\mathrm{E}^{\prime}}\right)^{-1}\left(t^{2} / 2\right)\right) .
$$

Let us discuss the monotonicity properties of $t \mapsto \sigma_{t}^{*}$ according to those of $\mathrm{S}^{\prime}$.

1. Case of softening materials $\left(\mathrm{S}^{\prime \prime}>0\right)$. Then $\alpha \mapsto \mathrm{S}^{\prime}$ is increasing from $-\mathrm{E}_{0}^{2} / \mathrm{E}_{0}^{\prime}$ to a limit, say $\mathrm{S}^{\prime}\left(\alpha_{m}\right)$, when $\alpha$ grows from 0 to $\alpha_{m}$. Since $t \mapsto \alpha_{t}^{*}$ is increasing when $t \geq \varepsilon_{\mathrm{c}}$, we deduce from (24) that $t \mapsto \sigma_{t}^{*}$ is decreasing from $\sigma_{\mathrm{c}}$ to $\sigma_{m}$,

$$
\sigma_{m}=\sqrt{\frac{2 \mathrm{w}_{1}}{\mathrm{~S}^{\prime}\left(\alpha_{m}\right)}},
$$


when $t$ grows from $\varepsilon_{c}$ to $\infty$. This property reflects the softening character of the damage model. Note that under Hypothesis 1, the stress $\sigma_{t}^{*}$ tends only asymptotically to $\sigma_{m}$ (which is not necessarily equal to 0 ). In other words, an infinite displacement is necessary to break the bar in the case of a homogeneous response.

2. Case of hardening materials $\left(\mathrm{S}^{\prime \prime}<0\right)$. Then $\alpha \mapsto \mathrm{S}^{\prime}$ is decreasing from $-\mathrm{E}_{0}^{2} / \mathrm{E}_{0}^{\prime}$ to $\mathrm{S}^{\prime}\left(\alpha_{m}\right) \geq 0$, when $\alpha$ grows from 0 to $\alpha_{m}$. Since $t \mapsto \alpha_{t}^{*}$ is increasing when $t \geq \varepsilon_{\mathrm{c}}$, we deduce from (24) that $t \mapsto \sigma_{t}^{*}$ is increasing from $\sigma_{\mathrm{c}}$ to $\sigma_{m}$ (still given by (26)) when $t$ grows from $\varepsilon_{\mathrm{c}}$ to $\infty$. This property reflects the hardening character of the damage model. The limit stress $\sigma_{m}$ is finite if and only if $\mathbf{S}^{\prime}\left(\alpha_{m}\right) \neq 0$.

Example 3. In the case of the family of damage laws of Example 1, the homogeneous evolution reads as

$$
u_{t}^{*}=t x, \quad \alpha_{t}^{*}=\left\{\begin{array}{ll}
0 & \text { if } t<\varepsilon_{\mathrm{c}} \\
1-\left(\frac{t}{\varepsilon_{\mathrm{c}}}\right)^{\frac{2}{1-q}} & \text { if } t \geq \varepsilon_{\mathrm{c}}
\end{array} \quad \text { with } \quad \varepsilon_{\mathrm{c}}=\frac{\sigma_{\mathrm{c}}}{\mathrm{E}_{0}} .\right.
$$

In a stress-strain diagram, the response of (any point of) the bar is given by

$$
\sigma=\left\{\begin{array}{ll}
\mathrm{E}_{0} \varepsilon & \text { if } \varepsilon<\varepsilon_{\mathrm{c}} \\
\mathrm{F}(\varepsilon) & \text { if } \varepsilon \geq \varepsilon_{\mathrm{c}}
\end{array}, \quad \mathrm{F}(\varepsilon)=\left(\frac{\varepsilon}{\varepsilon_{\mathrm{c}}}\right)^{\frac{1+q}{1-q}} \sigma_{\mathrm{c}} .\right.
$$

So, $\varepsilon \mapsto \mathrm{F}(\varepsilon)$ is a power law whose exponent $r, r=(1+q) /(1-q)$, only depends on the parameter $q>1$, see Figure 1(i). That means that all the models of this family with the same ratio $q$ lead to the same homogeneous response and hence are indistinguishable by such a test. However, the models with the same $q$ differ by the stability properties of the homogeneous response as it is shown in the next subsection. The exponent $r$ goes from -1 to $-\infty: r=-1$ corresponds to the limit case where $q=+\infty$ and where the dissipated energy up to failure is still infinite; $r=-\infty$ corresponds to the limit case where $q=1$ and where the strain remains equal to $\varepsilon_{\mathrm{c}}$ during the damage process (limit of a snap-back behavior).

Example 4. In the case of the family of damage laws of Example 2, the homogeneous evolution reads as

$$
u_{t}^{*}=t x, \quad \alpha_{t}^{*}=\left\{\begin{array}{ll}
0 & \text { if } t<\varepsilon_{\mathrm{c}} \\
\left(\frac{t}{\varepsilon_{\mathrm{c}}}\right)^{\frac{2}{1+q}}-1 & \text { if } t \geq \varepsilon_{\mathrm{c}}
\end{array} \quad \text { with } \quad \varepsilon_{\mathrm{c}}=\frac{\sigma_{\mathrm{c}}}{\mathrm{E}_{0}} .\right.
$$

In a stress-strain diagram, the response of (any point of) the bar is given by

$$
\sigma=\left\{\begin{array}{ll}
\mathrm{E}_{0} \varepsilon & \text { if } \varepsilon<\varepsilon_{\mathrm{c}} \\
\mathrm{F}(\varepsilon) & \text { if } \varepsilon \geq \varepsilon_{\mathrm{c}}
\end{array}, \quad \mathrm{F}(\varepsilon)=\left(\frac{\varepsilon}{\varepsilon_{\mathrm{c}}}\right)^{\frac{1-q}{1+q}} \sigma_{\mathrm{c}}\right.
$$

So, $\varepsilon \mapsto \mathrm{F}(\varepsilon)$ is a power law whose exponent $r=(1-q) /(1+q)$ only depends on the parameter $q>0$, see Figure 1(ii). As in the previous example, all the models of this family with the same $q$ give place to the same homogeneous response and hence are indistinguishable by such a test. But 
again, the models with the same $q$ differ by the stability properties of the homogeneous response. The exponent $r$ goes from 1 to $-1: r=1$ corresponds to the limit case where $q=0$ and where the behavior is purely elastic, while $r=-1$ corresponds to the limit case where $q=+\infty$ and where the dissipated energy up to failure is infinite. The behavior is with hardening if $0<q<1$ and with softening if $q>1$.

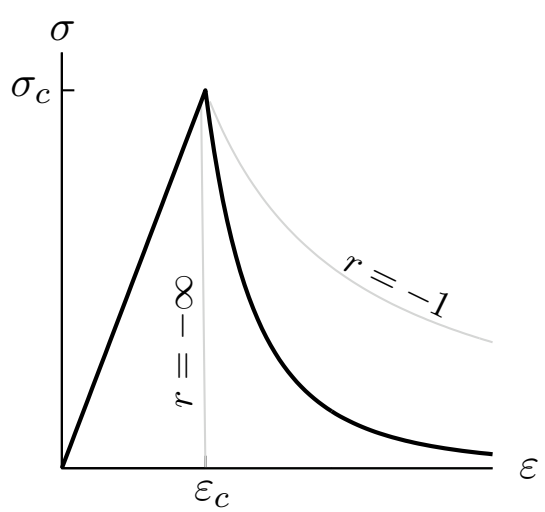

(i) strongly brittle

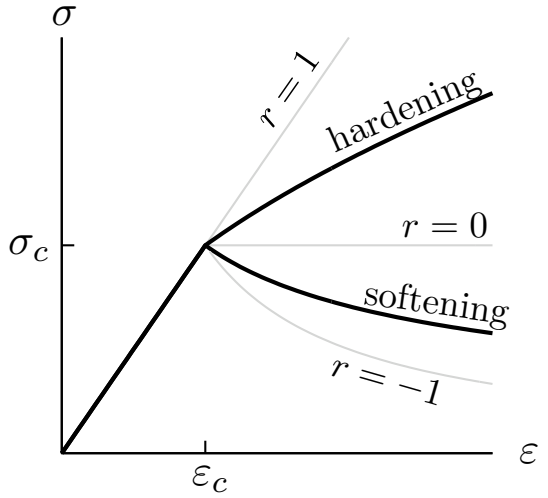

(ii) weakly brittle

Figure 1: The stress-strain responses associated with generic models of the families of Examples 1 and 2: (i) one example of a strongly brittle material for which the energy necessary to break the material is finite; (ii) two examples of weakly brittle materials for which the energy necessary to break the material is infinite, one with hardening and the other with softening. The gray lines correspond to the limit cases of the exponent $r$ of the power law.

\subsection{Energetic interpretations}

Let us interpret the homogeneous evolution in energetic terms. Let $\mathcal{W}_{t}$ be the work done by the external forces during the evolution up to time $t$ and let $\mathcal{P}_{t}$ be the elastic energy stored in the bar at time $t$. By definition, we have

$$
\begin{aligned}
\mathcal{W}_{t} & =\int_{0}^{t} \sigma_{\tau}^{*} \dot{U}_{\tau} d \tau=L \int_{0}^{t} \sigma_{\tau}^{*} d \tau \\
\mathcal{P}_{t} & =\int_{0}^{L} \frac{1}{2} \mathrm{E}\left(\alpha_{t}^{*}\right)\left(\frac{\partial u_{t}^{*}}{\partial x}\right)^{2} d x=\frac{1}{2} \mathrm{E}\left(\alpha_{t}^{*}\right) t^{2} L .
\end{aligned}
$$

Let $\mathcal{D}_{t}$ be the dissipated energy during the evolution up to time $t, \mathcal{D}_{t}$ is defined by

$$
\mathcal{D}_{t}=\mathcal{W}_{t}-\mathcal{P}_{t}
$$

Let us prove that

$$
\mathcal{D}_{t}=\mathrm{w}_{1} \alpha_{t}^{*} L
$$


Indeed, it is true at $t=0$. Then, differentiating (33) and using the definition of $\sigma_{t}^{*},(22)$ and (31) lead to

$$
\dot{\mathcal{D}}_{t}=\sigma_{t}^{*} L-\mathrm{E}\left(\alpha_{t}^{*}\right) t L-\frac{1}{2} \mathrm{E}^{\prime}\left(\alpha_{t}^{*}\right) t^{2} \dot{\alpha}_{t}^{*} L=\mathrm{w}_{1} \dot{\alpha}_{t}^{*} L .
$$

Integrating in time gives the desired result. (The reader is invited to verify that the property (34) holds true even if the loading is not monotonically increasing.) That allows us to interpret the material parameter $w_{1}$ as the dissipated energy (by unit length) during a process where the damage of the "volume element" grows from 0 to 1 . In particular, in the case of a strongly brittle material, i.e. when $\alpha_{m}=1, \mathrm{w}_{1}$ is the total energy dissipated to break the "volume element". This property gives a method to identify the material parameter $w_{1}$ as well as the constitutive function $\alpha \mapsto \mathrm{E}(\alpha)$ from experimental results based on the stress-strain response. It is one of the ingredients that we will use in Section 5 to identify the model from uniaxial tests. Let us note however that the constitutive function $\alpha \mapsto \mathrm{L}(\alpha)$ has no influence on the stress-strain response associated with the homogeneous evolution. As detailed in Section 5, additional measurements are necessary to identify $\alpha \mapsto \mathrm{L}(\alpha)$. Since $\mathcal{W}_{t}=\mathrm{W}\left(t, \alpha_{t}^{*}, 0\right) L, \mathrm{~W}$ can be interpreted as the state function giving the strain work by unit length.

Remark 4. In the case of weakly brittle damage models, by definition, the (density of) energy necessary to break a volume element is infinite. It is also the case to break a body (in our case, a bar) of finite size by a homogeneous damage process. But, when the size of the body is large enough, such a homogeneous damage process is no more stable (see the next subsection) and the body will break after a localized damage process. In such a case the corresponding fracture energy is not necessarily infinite, that depends on the parameters of the models. This question is not addressed in the paper because that requires to make a careful analysis of localized solutions, see however Pham et al. (2010).

Notation 1. From now on, we will use the following simplified notation for quantities referring to the homogeneous evolution:

$$
\begin{gathered}
\mathrm{E}_{t}=\mathrm{E}\left(\alpha_{t}^{*}\right), \mathrm{E}_{t}^{\prime}=\mathrm{E}^{\prime}\left(\alpha_{t}^{*}\right), \mathrm{E}_{t}^{\prime \prime}=\mathrm{E}^{\prime \prime}\left(\alpha_{t}^{*}\right), \mathrm{S}_{t}=\mathrm{S}\left(\alpha_{t}^{*}\right), \mathrm{S}_{t}^{\prime}=\mathrm{S}^{\prime}\left(\alpha_{t}^{*}\right), \mathrm{S}_{t}^{\prime \prime}=\mathrm{S}^{\prime \prime}\left(\alpha_{t}^{*}\right), \\
\Sigma_{t}=\sigma_{t}^{*}=\mathrm{E}\left(\alpha_{t}^{*}\right) t, \quad \mathrm{~L}_{t}=\mathrm{L}\left(\alpha_{t}^{*}\right)
\end{gathered}
$$

\subsection{Analysis of the stability of a homogeneous state}

By construction, at each time of the evolution, the homogeneous state satisfies the first order stability condition (st). It remains to see whether it satisfies the full stability condition (ST). To this end, at given $t>0$, let us develop the total energy of the perturbing state $\left(u_{t}^{*}+h v, \alpha_{t}^{*}+h \beta\right)$ with respect to $h$ up to the second order for a given admissible direction $(v, \beta) \in \mathcal{C}^{0} \times \mathcal{D}_{+}$:

$$
\mathcal{E}\left(u_{t}^{*}+h v, \alpha_{t}^{*}+h \beta\right)=\mathcal{E}\left(u_{t}^{*}, \alpha_{t}^{*}\right)+h \mathcal{E}^{\prime}\left(u_{t}^{*}, \alpha_{t}^{*}\right)(v, \beta)+\frac{h^{2}}{2} \mathcal{E}^{\prime \prime}\left(u_{t}^{*}, \alpha_{t}^{*}\right)(v, \beta)+o\left(h^{2}\right) .
$$


In $(37), \mathcal{E}^{\prime \prime}\left(u_{t}^{*}, \alpha_{t}^{*}\right)$ denotes the second derivative of $\mathcal{E}$ at $\left(u_{t}^{*}, \alpha_{t}^{*}\right)$, i.e. the quadratic form defined on $H^{1}(0, L)^{2}$ by

$$
\mathcal{E}^{\prime \prime}\left(u_{t}^{*}, \alpha_{t}^{*}\right)(v, \beta)=\int_{0}^{L}\left(\mathrm{w}_{1} \mathrm{~L}_{t}^{2} \beta^{\prime 2}+\frac{1}{2} \mathrm{E}_{t}^{\prime \prime} t^{2} \beta^{2}+2 \mathrm{E}_{t}^{\prime} t v^{\prime} \beta+\mathrm{E}_{t} v^{\prime 2}\right) d x .
$$

By virtue of the equilibrium equation, the first order term is given by

$$
\mathcal{E}^{\prime}\left(u_{t}^{*}, \alpha_{t}^{*}\right)(v, \beta)=\left(\frac{1}{2} \mathrm{E}_{t}^{\prime} t^{2}+\mathrm{w}_{1}\right) \int_{0}^{L} \beta d x
$$

and using (20) we get

$$
\mathcal{E}^{\prime}\left(u_{t}^{*}, \alpha_{t}^{*}\right)(v, \beta)=\left\{\begin{array}{ll}
-\frac{1}{2} \mathrm{E}_{0}^{\prime}\left(\varepsilon_{\mathrm{c}}^{2}-t^{2}\right) \int_{0}^{L} \beta d x \geq 0 & \text { if } t<\varepsilon_{\mathrm{c}} \\
0 & \text { if } t \geq \varepsilon_{\mathrm{c}}
\end{array} .\right.
$$

Let us discuss the stability of the homogeneous state by discriminating the elastic phase and the damaging phase.

1. Elastic phase: $\mathbf{t}<\varepsilon_{\mathrm{c}}$. The homogeneous state is then $(t x, 0)$. The first order term in $(37)$ is positive in all directions $(v, \beta)$ such that $\beta \neq 0$. Accordingly, the homogeneous state is stable in such directions. In the other admissible directions $(v, 0), v \neq 0$, the first order term of (37) vanishes and the second order term is given by

$$
\mathcal{E}^{\prime \prime}(t x, 0)(v, 0)=\mathrm{E}_{0} \int_{0}^{L} v^{\prime 2} d x
$$

and hence is positive. Therefore the homogeneous state is also stable in those directions and hence in all admissible directions. Thus (ST) holds and we have proved the

Proposition 3.2. As long as $t<\varepsilon_{\mathrm{c}}$, the homogeneous (undamaged) response $(t x, 0)$ is solution of the evolution problem.

2. Damaging phase: $\mathbf{t} \geq \varepsilon_{c}$. The homogeneous state is then $\left(t x, \alpha_{t}^{*}\right)$ and the first order term in (37) vanishes. Therefore, the stability of the homogeneous state depends on the sign of the second derivative. Specifically, the homogeneous damaged state is stable if (resp. only if) $\mathcal{E}^{\prime \prime}\left(u_{t}^{*}, \alpha_{t}^{*}\right)(v, \beta)>0$ (resp. $\geq 0$ ) for every (non null) admissible direction. Introducing the stress $\Sigma_{t}=\mathrm{E}_{t} t$, recalling that $\mathrm{S}_{t}^{\prime} \Sigma_{t}^{2}=2 \mathrm{w}_{1}$ and using the equality $\mathrm{E}_{t}^{\prime \prime} t^{2}=2 \mathrm{E}_{t} \mathrm{~S}_{t}^{\prime 2} \Sigma_{t}^{2}-\mathrm{S}_{t}^{\prime \prime} \Sigma_{t}^{2}$, the second order term becomes

$$
\mathcal{E}^{\prime \prime}\left(u_{t}^{*}, \alpha_{t}^{*}\right)(v, \beta)=\mathrm{w}_{1} \mathrm{~L}_{t}^{2} \int_{0}^{L} \beta^{\prime 2} d x+\mathrm{E}_{t} \int_{0}^{L}\left(v^{\prime}-\mathrm{S}_{t}^{\prime} \Sigma_{t} \beta\right)^{2} d x-\frac{1}{2} \mathrm{~S}_{t}^{\prime \prime} \Sigma_{t}^{2} \int_{0}^{L} \beta^{2} d x .
$$

Let us discriminate now between hardening and softening behaviors.

(a) Case of hardening behavior. Then $\mathrm{S}_{t}^{\prime \prime}<0$ and we deduce from (40) that

$$
\mathcal{E}^{\prime \prime}\left(u_{t}^{*}, \alpha_{t}^{*}\right)(v, \beta) \geq 0, \quad \forall(v, \beta) \in \mathcal{C}^{0} \times \mathcal{D}_{+}
$$

with the equality to 0 if and only if $v=0$ and $\beta=0$. Therefore, the state $\left(u_{t}^{*}, \alpha_{t}^{*}\right)$ is stable and we have proved the following property 
Proposition 3.3. In the case of a hardening behavior, the homogeneous evolution is solution of the evolution problem, i.e. satisfies (IR), (ST) and (EB) at all t.

(b) Case of softening behavior. Then $S_{t}^{\prime \prime}>0$. Let us note in (40) that the second order term is positive in any admissible direction of the form $(v, 0)$ with $v \neq 0$. It remains to study its sign for the other admissible directions, i.e. when $(v, \beta) \in \mathcal{C}^{0} \times \mathcal{D}_{+}, \beta \neq 0$. For such directions, the second directional derivative is the difference between the first two positive terms and the third positive term on the right hand side of (40). Then, the study of its sign is equivalent to compare the following Rayleigh ratio with 1 :

$$
\mathcal{R}_{t}(v, \beta)=\frac{\mathrm{w}_{1} \mathrm{~L}_{t}^{2} \int_{0}^{L} \beta^{\prime 2} d x+\mathrm{E}_{t} \int_{0}^{L}\left(v^{\prime}-\mathrm{S}_{t}^{\prime} \Sigma_{t} \beta\right)^{2} d x}{\frac{1}{2} \mathrm{~S}_{t}^{\prime \prime} \Sigma_{t}^{2} \int_{0}^{L} \beta^{2} d x} .
$$

Specifically, the stability of the homogeneous state is given by

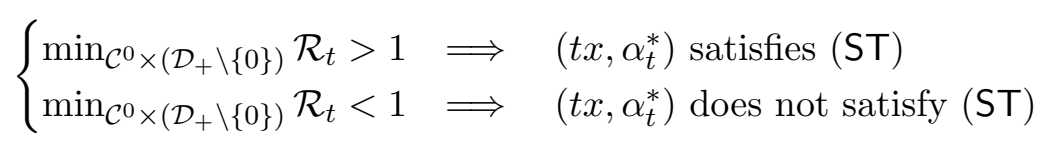

The minimum of the Rayleigh ratio is obtained in a closed form in Appendix A.2 (after the change of variable $x \mapsto x / L$ ) and we get

$$
\min _{\mathcal{C}^{0} \times\left(\mathcal{D}_{+} \backslash\{0\}\right)} \mathcal{R}_{t}= \begin{cases}\frac{2 \mathrm{E}_{t} \mathrm{~S}_{t}^{\prime 2}}{\mathrm{~S}_{t}^{\prime \prime}} & \text { if } \pi^{2} \mathrm{w}_{1} \mathrm{~L}_{t}^{2} \geq \mathrm{E}_{t} \mathrm{~S}_{t}^{\prime 2} \Sigma_{t}^{2} L^{2} \\ \frac{\left(\pi^{2} \mathrm{w}_{1}\right)^{1 / 3}\left(\mathrm{E}_{t} \mathrm{~S}_{t}^{\prime 2} \Sigma_{t}^{2}\right)^{2 / 3}}{\frac{1}{2} \mathrm{~S}_{t}^{\prime \prime} \Sigma_{t}^{2}}\left(\frac{\mathrm{L}_{t}}{L}\right)^{2 / 3} & \text { if } \pi^{2} \mathrm{w}_{1} \mathrm{~L}_{t}^{2}<\mathrm{E}_{t} \mathrm{~S}_{t}^{\prime 2} \Sigma_{t}^{2} L^{2}\end{cases}
$$

Let us note that $2 \mathrm{E}_{t} \mathrm{~S}_{t}^{\prime 2}>\mathrm{S}_{t}^{\prime \prime}$. Indeed, $2 \mathrm{E}_{t} \mathrm{~S}_{t}^{\prime 2}-\mathrm{S}_{t}^{\prime \prime}=\mathrm{E}_{t}^{\prime \prime} / \mathrm{E}_{t}^{2}$ and the inequality holds since $\mathrm{E}_{t}^{\prime \prime}>0$ by virtue of Hypothesis 1. Therefore, the homogeneous state is stable when $\pi^{2} \mathrm{w}_{1} \mathrm{~L}_{t}^{2} \geq$ $\mathrm{E}_{t} \mathrm{~S}_{t}^{\prime 2} \Sigma_{t}^{2} L^{2}$. On the other hand, when $\pi^{2} \mathrm{w}_{1} \mathrm{~L}_{t}^{2}<\mathrm{E}_{t} \mathrm{~S}_{t}^{\prime 2} \Sigma_{t}^{2} L^{2}$, the homogeneous state is stable provided that $\pi^{2} \mathrm{w}_{1}\left(\mathrm{E}_{t} \mathrm{~S}_{t}^{\prime 2} \Sigma_{t}^{2}\right)^{2} \mathrm{~L}_{t}^{2}>\left(\frac{1}{2} \mathrm{~S}_{t}^{\prime \prime} \Sigma_{t}^{2}\right)^{3} L^{2}$. We have thus proved

Proposition 3.4. For $t \geq \varepsilon_{\mathrm{c}}$, the homogeneous state $\left(t x, \alpha_{t}^{*}\right)$ is stable if and only if the length of the bar is sufficiently small. Specifically, we have

$$
\left\{\begin{array}{lll}
L<\mathrm{L}_{\mathrm{s}}(t) & \Longrightarrow & \left(\text { tx, } \alpha_{t}^{*}\right) \text { satisfies }(\mathrm{ST}) \\
L>\mathrm{L}_{\mathrm{s}}(t) & \Longrightarrow & \left(\text { tx }, \alpha_{t}^{*}\right) \text { does not satisfy }(\mathrm{ST})
\end{array}\right.
$$

with

$$
\mathrm{L}_{\mathbf{s}}(t)=\sqrt{\frac{8 \pi^{2} \mathrm{~S}_{t}^{\prime 4} \mathrm{~W}_{1}}{\mathrm{~S}_{t}^{\prime \prime 3} t^{2}}} \mathrm{~L}_{t}
$$

Accordingly, each homogeneous state is stable when the length of the bar is less than a critical value and unstable otherwise. (Note however that the case $L=\mathrm{L}_{\mathrm{s}}(t)$ needs a refinement of 
the proof to determine whether the homogeneous state is stable. Indeed, in such a case the minimum of the Rayleigh ratio is equal to 1 and the stability will depend on the higher order derivatives of the constitutive functions. This study is outside the scope of our paper.) The critical value of the bar length is proportional to the internal length of the material and the coefficient of proportionality depends on the state. This dependence is a characteristic of the material and is a byproduct of the two constitutive functions $\alpha \mapsto \mathrm{E}(\alpha)$ and $\alpha \mapsto \mathrm{L}(\alpha)$. The example below illustrates this dependence for the families of models of Examples 1 and 2.

Example 5. In the case of the family of models of Example 1, after some straightforward calculations which use (27) and (28), we get that the critical length of the bar beyond which the homogeneous state $\left(\varepsilon x, \alpha_{\varepsilon}^{*}\right)$ is no more stable is given by

$$
\mathrm{L}_{\mathrm{s}}(\varepsilon)=\left(\frac{\varepsilon}{\varepsilon_{\mathrm{c}}}\right)^{\frac{2 p+1}{1-q}} \ell_{\mathrm{c}}^{\mathrm{s}}, \quad \ell_{\mathrm{c}}^{\mathrm{s}}=\frac{2 \pi q}{(q+1)^{3 / 2}} \ell_{\mathrm{c}} .
$$

Thus, contrarily to the stress-strain response, the loss of stability of the homogeneous state depends on both coefficients $p \in \mathbb{R}$ and $q>1$. When $p \neq-\frac{1}{2}, \mathrm{~L}_{\mathbf{s}}$ is monotonic and its inverse $\varepsilon_{\mathbf{s}}$ is given by

$$
\varepsilon_{\mathbf{s}}(L)=\left(\frac{L}{\ell_{\mathrm{c}}^{\mathbf{s}}}\right)^{\frac{1-q}{2 p+1}} \varepsilon_{\mathrm{c}}, \quad p \neq-\frac{1}{2} .
$$

The dependence of $\mathrm{L}_{\mathrm{s}}$ on the strain $\varepsilon$ leads to the following types of responses according to the length of the bar and the value of $p$ :

1. When $p>-\frac{1}{2}, \mathrm{~L}_{\mathrm{s}}$ is a decreasing function of $\varepsilon$. Therefore, all homogeneous states beyond the critical strain $\varepsilon_{\mathrm{c}}$ are unstable when $L>\ell_{\mathrm{c}}^{\mathrm{s}}$, while only the homogeneous states such that $\varepsilon_{\mathrm{c}} \leq \varepsilon<\varepsilon_{\mathbf{s}}(L)$ are stable when $L<\ell_{\mathrm{c}}^{\mathrm{s}}$. Accordingly, if we assume that the evolution will follow the homogeneous branch as long as the state is stable, we will observe the following type of response during a test where $\varepsilon$ is increasing $(\varepsilon=t)$, see Figure 2(i):

(a) If $L<\ell_{\mathrm{c}}^{\mathrm{s}}$, then the evolution of the bar follows the homogeneous branch as long as $\varepsilon<\varepsilon_{\mathbf{s}}(L)$, but will bifurcate (or jump) to a non homogeneous branch when $\varepsilon=\varepsilon_{\mathbf{s}}(L)$.

(b) If $L>\ell_{\mathrm{c}}^{\mathrm{s}}$, then the state of the bar is no more homogeneous as soon as $\varepsilon$ becomes greater than the critical strain $\varepsilon_{\mathrm{c}}$.

2. When $p=-\frac{1}{2}, \mathrm{~L}_{\mathrm{s}}$ is the constant $\ell_{\mathrm{c}}^{\mathrm{s}}$. All homogeneous states are stable if $L<\ell_{\mathrm{c}}^{\mathrm{s}}$ and all states beyond the critical strain $\varepsilon_{\mathrm{c}}$ are unstable if $L>\ell_{\mathrm{c}}^{\mathrm{s}}$, see Figure $2($ ii).

3. When $p<-\frac{1}{2}, \mathrm{~L}_{\mathrm{s}}$ is an increasing function of $\varepsilon$. All homogeneous states are stable when $L<\ell_{\mathrm{c}}^{\mathrm{s}}$, while only the homogeneous states such that $\varepsilon<\varepsilon_{\mathrm{c}}$ or $\varepsilon>\varepsilon_{\mathrm{s}}(L)$ are stable when $L>\ell_{\mathrm{c}}^{\mathrm{s}}$. That leads to the following type of response during a test where $\varepsilon$ is increasing $(\varepsilon=t)$, see Figure 2(iii):

(a) If $L<\ell_{c}^{\mathrm{s}}$, then the evolution of the bar follows the homogeneous branch for all $\varepsilon$.

(b) If $L>\ell_{\mathrm{c}}^{\mathrm{s}}$, then the evolution of the bar will bifurcate or jump to a non homogeneous branch as soon as $\varepsilon>\varepsilon_{\mathrm{c}}$. But, the evolution of the bar could recover the homogeneous branch when $\varepsilon>\varepsilon_{\mathbf{s}}(L)$ since the homogeneous states become stable again. 

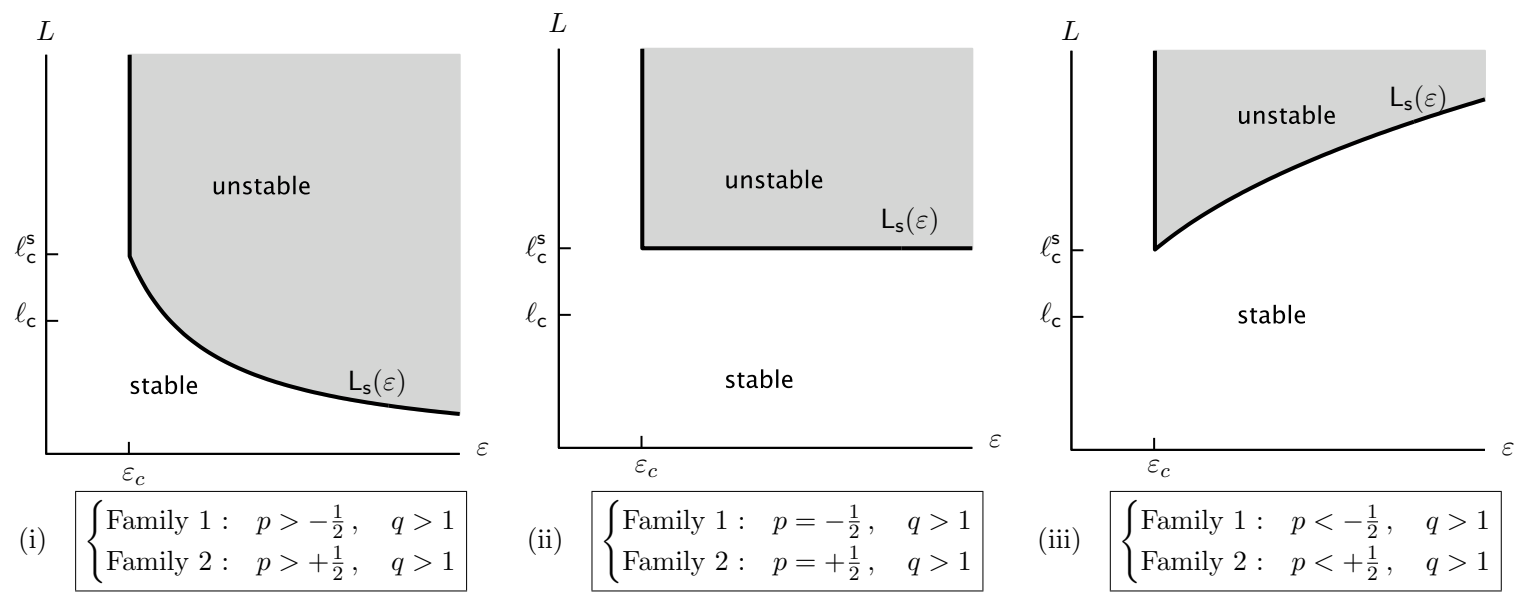

Figure 2: Domains of stable and unstable homogeneous states in a diagram $L-\varepsilon$ ( $L=$ length of the bar, $\varepsilon$ $=$ homogeneous strain) for a given $q$ and different values of $p$ for the families of strongly brittle materials of Example 1 or weakly brittle materials with softening of Example 2.

Example 6. In the case of weakly brittle materials with softening $(q>1)$ of Example 2, using (29) and (30), we get that the critical length of the bar beyond which the homogeneous state $\left(\varepsilon x, \alpha_{\varepsilon}^{*}\right)$ is no more stable is given by

$$
\mathrm{L}_{\mathrm{s}}(\varepsilon)=\left(\frac{\varepsilon}{\varepsilon_{\mathrm{c}}}\right)^{\frac{1-2 p}{q+1}} \ell_{\mathrm{c}}^{\mathrm{s}}, \quad \ell_{\mathrm{c}}^{\mathrm{s}}=\frac{2 \pi q}{(q-1)^{3 / 2}} \ell_{\mathrm{c}} .
$$

Thus, the loss of stability of the homogeneous state depends on both coefficients $p \in \mathbb{R}$ and $q>1$. When $p \neq \frac{1}{2}, \mathrm{~L}_{\mathrm{s}}$ is monotonic and its inverse $\varepsilon_{\mathbf{s}}$ is given by

$$
\varepsilon_{\mathbf{s}}(L)=\left(\frac{L}{\ell_{\mathrm{c}}^{\mathrm{s}}}\right)^{\frac{q+1}{1-2 p}} \varepsilon_{\mathrm{c}}, \quad p \neq \frac{1}{2} .
$$

The dependence of $\mathrm{L}_{\mathrm{s}}$ on the strain $\varepsilon$ leads to the same types of responses according to the length of the bar as for Example 5, see Figure 2.

\section{Bifurcation of the evolution from the homogeneous response}

The response of the bar can follow the homogeneous branch as long as the associated homogeneous state is stable. We have just seen that this is possible provided that the length of the bar and/or the strain are sufficiently small. But, the fact that the homogeneous branch is stable does not guaranty that the evolution will follow this branch up to its loss of stability. It can happen that the evolution bifurcates on another branch (necessarily associated with non homogeneous states), whenever such a branch exists and is itself stable (at least in a neighborhood of the 
bifurcation point). (It could even happen that the evolution jumps from a stable homogeneous state to another stable state; it is allowed in our formulation of the evolution problem, but we do not consider here such a case.) Accordingly, it is important to identify the possible points of bifurcation on the homogeneous branch. It is the aim of this section.

\subsection{Setting of the bifurcation problem}

Let $t>0$ be a given stretching and $\left(u_{t}^{*}, \alpha_{t}^{*}\right)$ be the associated state of the homogeneous branch, $\left(u_{t}^{*}, \alpha_{t}^{*}\right)$ is given by (20). Let us study the evolution problem in the time interval $[t, t+\eta)$, with $\eta>0$ and small enough, assuming that the state of the bar is the homogeneous one $\left(u_{t}^{*}, \alpha_{t}^{*}\right)$ at time $t$. Let $\left\{\left(u_{\tau}, \alpha_{\tau}\right)\right\}_{\tau \in[t, t+\eta)}$ be a possible solution of the evolution problem during the time interval $[t, t+\eta)$. The evolution has to satisfy (IR), (ST) (hence (st)) and (EB). Let us assume that the evolution is sufficiently smooth so that the right derivative exists at $t$. This derivative is denoted $(\dot{u}, \dot{\alpha})$ and is defined by

$$
\dot{u}=\lim _{\tau \downarrow t} \frac{u_{\tau}-u_{t}^{*}}{\tau-t}, \quad \dot{\alpha}=\lim _{\tau \downarrow t} \frac{\alpha_{\tau}-\alpha_{t}^{*}}{\tau-t} .
$$

Of course, $(\dot{u}, \dot{\alpha})=\left(x, \dot{\alpha}_{t}^{*}\right)$ if the evolution follows the homogeneous branch. Our purpose is to find whether another evolution rate is possible. Let us deduce from the three items of the evolution problem a set of necessary conditions that $(\dot{u}, \dot{\alpha})$ must satisfy. We discriminate the case $t<\varepsilon_{\mathrm{c}}$ and the case $t \geq \varepsilon_{\mathrm{c}}$.

1. Case $t<\varepsilon_{\mathrm{c}}$. Then the bar is undamaged and $\left(u_{t}^{*}, \alpha_{t}^{*}\right)=(t x, 0)$. The three items (IR), (st) and (EB) give the following necessary conditions for $(\dot{u}, \dot{\alpha})$ :

(a) By (IR), we get $\dot{\alpha} \geq 0$;

(b) The energy balance (EB) leads to (eb) which at time $t$ reads as

$$
0=\left(-\frac{1}{2} \mathrm{~S}_{0}^{\prime} \Sigma_{t}^{2}+\mathrm{w}_{1}\right) \int_{0}^{L} \dot{\alpha} d x
$$

Since $t<\varepsilon_{\mathrm{c}}, \mathrm{w}_{1}>\frac{1}{2} \mathrm{~S}_{0}^{\prime} \Sigma_{t}^{2}$ and hence $\int_{0}^{L} \dot{\alpha} d x=0$. By virtue of (IR), this last equality is possible if and only if $\dot{\alpha}(x)=0=\dot{\alpha}_{t}^{*}$.

(c) The stability condition (ST) implies the first order stability condition (st) which, in turn, implies (13). Taking the right derivative of (13) at time $t$ and using $\dot{\alpha}=0$ give $\dot{\sigma}=\mathrm{E}_{0}, \dot{\sigma}$ denoting the right derivative of $\sigma_{\tau}$ at $t$. Since $u_{\tau}^{\prime}(x)=\mathrm{S}\left(\alpha_{\tau}(x)\right) \sigma_{\tau}$, we obtain $\dot{u}^{\prime}(x)=1$ and hence $\dot{u}(x)=x=\dot{u}_{t}^{*}(x)$.

We have thus proved the

Proposition 4.1. When $t<\varepsilon_{\mathrm{c}}$, the unique possible evolution rate is that of the homogeneous response: $(\dot{u}, \dot{\alpha})=(x, 0)$. That means that there is no possibility of bifurcation from the homogeneous branch as long as the bar is undamaged. 
2. Case $t \geq \varepsilon_{c}$. Then the homogeneous state is $\left(t x, \alpha_{t}^{*}\right)$ with $\alpha_{t}^{*} \in\left[0, \alpha_{m}\right)$ given by $-\mathrm{E}^{\prime}\left(\alpha_{t}^{*}\right) t^{2}=$ $2 \mathrm{w}_{1}$. The three items (IR), (st) and (EB) give the following necessary conditions for $(\dot{u}, \dot{\alpha})$ :

(a) By (IR), we get $\dot{\alpha} \geq 0$ again and hence $(\dot{u}, \dot{\alpha}) \in \mathcal{C}_{1} \times \mathcal{D}_{+}$;

(b) The stability condition (ST) implies the first order stability condition (st) which at time $t+h$ reads as

$$
\forall(v, \beta) \in \mathcal{C}^{0} \times \mathcal{D}_{+}, \quad \mathcal{E}^{\prime}\left(u_{t+h}, \alpha_{t+h}\right)(v, \beta) \geq 0 .
$$

But since $\mathcal{E}^{\prime}\left(u_{t}^{*}, \alpha_{t}^{*}\right)(v, \beta)=0$ (see (39)), dividing the inequality above by $h$ and passing to the limit when $h$ goes to 0 give the following inequality that the evolution rate must satisfy

$$
\forall(v, \beta) \in \mathcal{C}^{0} \times \mathcal{D}_{+}, \quad \mathcal{E}_{t}^{\prime \prime}((\dot{u}, \dot{\alpha}),(v, \beta)) \geq 0 .
$$

In $(50), \mathcal{E}_{t}^{\prime \prime}$ denotes the second directional derivative of $\mathcal{E}$ at $\left(u_{t}^{*}, \alpha_{t}^{*}\right)$ considered as the symmetric bilinear form defined on $H^{1}(0, L)^{2}$ by

$$
\mathcal{E}_{t}^{\prime \prime}((\dot{u}, \dot{\alpha}),(v, \beta))=\int_{0}^{L}\left(\mathrm{w}_{1} \mathrm{~L}_{t}^{2} \dot{\alpha}^{\prime} \beta^{\prime}+\mathrm{E}_{t}\left(\dot{u}^{\prime}-\mathrm{S}_{t}^{\prime} \Sigma_{t} \dot{\alpha}\right)\left(v^{\prime}-\mathrm{S}_{t}^{\prime} \Sigma_{t} \beta\right)-\frac{1}{2} \mathrm{~S}_{t}^{\prime \prime} \Sigma_{t}^{2} \dot{\alpha} \beta\right) d x
$$

Considering first (50) with $\beta=0$ and using the fact that $\mathcal{C}^{0}$ is a linear space, we get

$$
\int_{0}^{L} \mathrm{E}_{t}\left(\dot{u}^{\prime}-\mathrm{S}_{t}^{\prime} \Sigma_{t} \dot{\alpha}\right) v^{\prime} d x=0, \quad \forall v \in \mathcal{C}^{0} .
$$

That leads to $\dot{u}^{\prime}(x)=\mathrm{S}_{t}^{\prime} \Sigma_{t} \dot{\alpha}(x)+\dot{C}$ where $\dot{C}$ is a constant. The constant is given by the boundary conditions $\dot{u}(0)=0$ and $\dot{u}(L)=L$. Finally, we obtain

$$
\dot{u}(x)=\left(1-\mathrm{S}_{t}^{\prime} \Sigma_{t}\langle\dot{\alpha}\rangle\right) x+\mathrm{S}_{t}^{\prime} \Sigma_{t} \int_{0}^{x} \dot{\alpha}(y) d y, \quad\langle\dot{\alpha}\rangle:=\frac{1}{L} \int_{0}^{L} \dot{\alpha}(y) d y .
$$

Inserting (53) into (50) leads to the following variational inequality for $\dot{\alpha}$ :

$$
\mathrm{w}_{1} \mathrm{~L}_{t}^{2} \int_{0}^{L} \dot{\alpha}^{\prime} \beta^{\prime} d x+\mathrm{E}_{t} \mathrm{~S}_{t}^{\prime 2} \Sigma_{t}^{2}\langle\dot{\alpha}\rangle \int_{0}^{L} \beta d x-\frac{1}{2} \mathrm{~S}_{t}^{\prime \prime} \Sigma_{t}^{2} \int_{0}^{L} \dot{\alpha} \beta d x \geq \mathrm{E}_{t} \mathrm{~S}_{t}^{\prime} \Sigma_{t} \int_{0}^{L} \beta d x
$$

which must hold for all $\beta \in \mathcal{D}_{+}$.

(c) The energy balance (EB) reads at time $t+h$

$$
\mathcal{E}\left(u_{t+h}, \alpha_{t+h}\right)=\mathcal{E}\left(u_{t}^{*}, \alpha_{t}^{*}\right)+L \int_{t}^{t+h} \sigma_{\tau} d \tau, \quad \sigma_{\tau}=\frac{\tau L}{\int_{0}^{L} \mathrm{~S}\left(\alpha_{\tau}(x)\right) d x}
$$

where we have taken into account that the stress is necessarily uniform in the bar at each time of a real evolution, see (13). Expanding $\mathcal{E}\left(u_{t+h}, \alpha_{t+h}\right)$ up to the second order and inserting into (55) give

$$
0=\mathcal{E}^{\prime}\left(u_{t}^{*}, \alpha_{t}^{*}\right)\left(\chi_{t+h}-\chi_{t}^{*}\right)+\frac{1}{2} \mathcal{E}^{\prime \prime}\left(u_{t}^{*}, \alpha_{t}^{*}\right)\left(\chi_{t+h}-\chi_{t}^{*}\right)-L \int_{t}^{t+h} \sigma_{\tau} d \tau+o\left(\left\|\chi_{t+h}-\chi_{t}^{*}\right\|^{2}\right)
$$


where $\chi_{t+h}=\left(u_{t+h}, \alpha_{t+h}\right), \chi_{t}^{*}=\left(u_{t}^{*}, \alpha_{t}^{*}\right)$ and $\|\cdot\|$ denotes the natural norm on $H^{1}(0, L)^{2}$. Since $\left(u_{t+h}-u_{t+h}^{*}\right) \in \mathcal{C}^{0}$ and $\left(\alpha_{t+h}-\alpha_{t}^{*}\right) \in \mathcal{D}_{+}$, we have

$$
\mathcal{E}^{\prime}\left(u_{t}^{*}, \alpha_{t}^{*}\right)\left(u_{t+h}-u_{t+h}^{*}, \alpha_{t+h}-\alpha_{t}^{*}\right)=0
$$

by virtue of (39). Therefore, using the definition (12) of $\mathcal{E}^{\prime}$, the boundary conditions $u_{t+h}^{*}(0)-u_{t}^{*}(0)=0$ and $u_{t+h}^{*}(L)-u_{t}^{*}(L)=h L$, the "initial" condition $\sigma_{t}=\Sigma_{t}$ and the equality $-\mathrm{E}^{\prime}\left(\alpha_{t}^{*}\right) t^{2}=2 \mathrm{w}_{1}$, we eventually get

$$
\mathcal{E}^{\prime}\left(u_{t}^{*}, \alpha_{t}^{*}\right)\left(\chi_{t+h}-\chi_{t}^{*}\right)=\mathcal{E}^{\prime}\left(u_{t}^{*}, \alpha_{t}^{*}\right)\left(u_{t+h}^{*}-u_{t}^{*}, 0\right)=h \sigma_{t} L .
$$

Then, (56) can read as

$$
0=\frac{1}{2} \mathcal{E}^{\prime \prime}\left(u_{t}^{*}, \alpha_{t}^{*}\right)\left(\chi_{t+h}-\chi_{t}^{*}\right)-L \int_{t}^{t+h}\left(\sigma_{\tau}-\sigma_{t}\right) d \tau+o\left(\left\|\chi_{t+h}-\chi_{t}^{*}\right\|^{2}\right) .
$$

Dividing (57) by $h^{2}$ and passing to the limit when $h$ goes to 0 , we finally obtain that the evolution rate must satisfy $\mathcal{E}^{\prime \prime}\left(u_{t}^{*}, \alpha_{t}^{*}\right)(\dot{u}, \dot{\alpha})=\dot{\sigma} L$ where $\dot{\sigma}$ denotes the right derivative of the stress at $t$. The second derivative of the energy can be calculated either with (38) or (51) while $\dot{\sigma}$ is obtained from (13). After elementary calculations using (53) we obtain

$$
\begin{aligned}
\mathcal{E}^{\prime \prime}\left(u_{t}^{*}, \alpha_{t}^{*}\right)(\dot{u}, \dot{\alpha}) & =\mathrm{w}_{1} \mathrm{~L}_{t}^{2} \int_{0}^{L} \dot{\alpha}^{2} d x+\mathrm{E}_{t}\left(1-\mathrm{S}_{t}^{\prime} \Sigma_{t}\langle\dot{\alpha}\rangle\right)^{2} L-\frac{1}{2} \mathrm{~S}_{t}^{\prime \prime} \Sigma_{t}^{2} \int_{0}^{L} \dot{\alpha}^{2} d x \\
\dot{\sigma} L & =\mathrm{E}_{t}\left(1-\mathrm{S}_{t}^{\prime} \Sigma_{t}\langle\dot{\alpha}\rangle\right) L .
\end{aligned}
$$

Therefore, $\dot{\alpha}$ must satisfy

$$
\mathrm{w}_{1} \mathrm{~L}_{t}^{2} \int_{0}^{L} \dot{\alpha}^{2} d x+\mathrm{E}_{t} \mathrm{~S}_{t}^{\prime 2} \Sigma_{t}^{2}\langle\dot{\alpha}\rangle^{2} L-\frac{1}{2} \mathrm{~S}_{t}^{\prime \prime} \Sigma_{t}^{2} \int_{0}^{L} \dot{\alpha}^{2} d x=\mathrm{E}_{t} \mathrm{~S}_{t}^{\prime} \Sigma_{t}\langle\dot{\alpha}\rangle L .
$$

We are in a position to set the bifurcation problem.

Proposition 4.2. At $t \geq \varepsilon_{c}$, the evolution rate $(\dot{u}, \dot{\alpha})$ of any branch which is solution of the evolution problem and passes through the homogeneous state $\left(u_{t}^{*}, \alpha_{t}^{*}\right)$ at $t$, is such that

$$
\begin{array}{r}
\dot{u}(x)=\left(1-\mathrm{S}_{t}^{\prime} \Sigma_{t}\langle\dot{\alpha}\rangle\right) x+\mathrm{S}_{t}^{\prime} \Sigma_{t} \int_{0}^{x} \dot{\alpha}(y) d y \\
\dot{\alpha} \in \mathcal{D}_{+}, \quad \mathcal{A}_{t}(\dot{\alpha}, \beta-\dot{\alpha}) \geq \mathcal{L}_{t}(\beta-\dot{\alpha}) \quad \forall \beta \in \mathcal{D}_{+} .
\end{array}
$$

In (61), $\mathcal{A}_{t}$ and $\mathcal{L}_{t}$ are respectively the symmetric bilinear form and the linear form defined on $H^{1}(0, L)$ by

$$
\begin{aligned}
\mathcal{A}_{t}(\alpha, \beta) & =\mathrm{w}_{1} \mathrm{~L}_{t}^{2} \int_{0}^{L} \alpha^{\prime} \beta^{\prime} d x+\mathrm{E}_{t} \mathrm{~S}_{t}^{2} \Sigma_{t}^{2} \frac{1}{L} \int_{0}^{L} \alpha d x \int_{0}^{L} \beta d x-\frac{1}{2} \mathrm{~S}_{t}^{\prime \prime} \Sigma_{t}^{2} \int_{0}^{L} \alpha \beta d x \\
\mathcal{L}_{t}(\beta) & =\mathrm{E}_{t} \mathrm{~S}_{t}^{\prime} \Sigma_{t} \int_{0}^{L} \beta d x .
\end{aligned}
$$


Proof. Let us remark that (54) and (59) read respectively as

$$
\mathcal{A}_{t}(\dot{\alpha}, \beta) \geq \mathcal{L}_{t}(\beta), \quad \forall \beta \in \mathcal{D}_{+} \quad \text { and } \quad \mathcal{A}_{t}(\dot{\alpha}, \dot{\alpha})=\mathcal{L}_{t}(\dot{\alpha})
$$

Substracting the equality to the inequality gives (61). Conversely, making $\beta=\dot{\alpha} / 2$ and $\beta=2 \dot{\alpha}$ in (61) leads to (59). Inserting (59) into (61) gives (54).

\subsection{Possible bifurcation points}

The homogeneous evolution rate $\dot{\alpha}_{t}^{*}(x)=-\frac{2 \mathrm{E}_{t}^{\prime}}{t \mathrm{E}_{t}^{\prime \prime}}$ is always solution of (61). The question is to know whether another solution exists. The uniqueness is guaranteed when the bilinear form $\mathcal{A}_{t}$ is positive definite on $H^{1}(0, L)$, i.e. when $\mathcal{A}_{t}(\beta, \beta)>0$ for all $\beta \in H^{1}(0, L) \backslash\{0\}$. Indeed, in such a case, let us consider another solution $\dot{\alpha}$. Making $\beta=\dot{\alpha}_{t}^{*}$ in (61) we obtain $\mathcal{A}_{t}\left(\dot{\alpha}, \dot{\alpha}_{t}^{*}-\dot{\alpha}\right) \geq \mathcal{L}_{t}\left(\dot{\alpha}_{t}^{*}-\dot{\alpha}\right)$. Making $\beta=\dot{\alpha}$ in the variational inequality satisfied by $\dot{\alpha}_{t}^{*}$, we get $\mathcal{A}_{t}\left(\dot{\alpha}_{t}^{*}, \dot{\alpha}-\dot{\alpha}_{t}^{*}\right) \geq \mathcal{L}_{t}\left(\dot{\alpha}-\dot{\alpha}_{t}^{*}\right)$. The addition of the two inequalities leads to $\mathcal{A}_{t}\left(\dot{\alpha}-\dot{\alpha}_{t}^{*}, \dot{\alpha}-\dot{\alpha}_{t}^{*}\right) \leq 0$ which is possible only if $\dot{\alpha}=\dot{\alpha}_{t}^{*}$.

It is clear according to its definition (62) that $\mathcal{A}_{t}$ is definite positive when $\mathrm{S}_{t}^{\prime \prime}<0$, i.e. in the case of a behavior with hardening. We have thus proved

Proposition 4.3. In the case of a hardening behavior, the unique possible evolution rate is that of the homogeneous response; there is no possibility of bifurcation from the homogeneous branch.

Remark 5. A stronger result should be to prove that the homogeneous response is the unique one when the behavior is with hardening. The proof is outside the scope of our paper.

Let us now consider softening behaviors, i.e. $\mathrm{S}_{t}^{\prime \prime}>0$. In such a case, the quadratic form associated with $\mathcal{A}_{t}$ contains two positive and one negative terms. Accordingly, the study of the positivity of $\mathcal{A}_{t}$ is equivalent to compare the following Rayleigh ratio $\hat{\mathcal{R}}_{t}$ with 1 :

$$
\hat{\mathcal{R}}_{t}(\beta)=\frac{\mathrm{w}_{1} \mathrm{~L}_{t}^{2} \int_{0}^{L} \beta^{\prime 2} d x+\mathrm{E}_{t} \mathrm{~S}_{t}^{\prime 2} \Sigma_{t}^{2} \frac{1}{L}\left(\int_{0}^{L} \beta d x\right)^{2}}{\frac{1}{2} \mathrm{~S}_{t}^{\prime \prime} \Sigma_{t}^{2} \int_{0}^{L} \beta^{2} d x}, \quad \beta \in H^{1}(0, L) \backslash\{0\} .
$$

Specifically, the possibility of bifurcation from the homogeneous state is given by

$$
\left\{\begin{array}{lll}
\min _{H^{1}(0, L) \backslash\{0\}} \hat{\mathcal{R}}_{t}>1 & \Longrightarrow & \text { no bifurcation } \\
\min _{H^{1}(0, L) \backslash\{0\}} \hat{\mathcal{R}}_{t} \leq 1 & \Longrightarrow & \text { bifurcation possible }
\end{array}\right.
$$

The minimum of the Rayleigh ratio is obtained in a closed form in Appendix A.1 (after the 
change of variable $x \mapsto x / L)$ and we get

$$
\min _{H^{1}(0, L) \backslash\{0\}} \hat{\mathcal{R}}_{t}= \begin{cases}\frac{2 \mathrm{E}_{t} \mathrm{~S}_{t}^{\prime 2}}{\mathrm{~S}_{t}^{\prime \prime}} & \text { if } \pi^{2} \mathrm{w}_{1} \mathrm{~L}_{t}^{2} \geq \mathrm{E}_{t} \mathrm{~S}_{t}^{\prime 2} \Sigma_{t}^{2} L^{2} \\ \frac{2 \pi^{2} \mathrm{w}_{1} \mathrm{~L}_{t}^{2}}{\mathrm{~S}_{t}^{\prime \prime} \Sigma_{t}^{2} L^{2}} & \text { if } \pi^{2} \mathrm{w}_{1} \mathrm{~L}_{t}^{2}<\mathrm{E}_{t} \mathrm{~S}_{t}^{\prime 2} \Sigma_{t}^{2} L^{2}\end{cases}
$$

But since $2 \mathrm{E}_{t} \mathrm{~S}_{t}^{\prime 2}>\mathrm{S}_{t}^{\prime \prime}$, no bifurcation is possible if $\pi^{2} \mathrm{w}_{1} \mathrm{~L}_{t}^{2} \geq \mathrm{E}_{t} \mathrm{~S}_{t}^{\prime 2} \Sigma_{t}^{2} L^{2}$. On the other hand, when $2 \pi^{2} \mathrm{w}_{1} \mathrm{~L}_{t}^{2} \leq \mathrm{S}_{t}^{\prime \prime} \Sigma_{t}^{2} L^{2}$, then $\pi^{2} \mathrm{w}_{1} \mathrm{~L}_{t}^{2}<\mathrm{E}_{t} \mathrm{~S}_{t}^{\prime 2} \Sigma_{t}^{2} L^{2}$ and a bifurcation is possible. We have thus proved

Proposition 4.4. For a softening material, when $t \geq \varepsilon_{c}$, a bifurcation from the homogeneous branch is possible if and only if the length of the bar is sufficiently large. Specifically, we have

$$
\left\{\begin{array}{lll}
L<\mathrm{L}_{\mathrm{b}}(t) & \Longrightarrow & \text { no bifurcation at }\left(u_{t}^{*}, \alpha_{t}^{*}\right) \\
L \geq \mathrm{L}_{\mathrm{b}}(t) & \Longrightarrow & \text { bifurcation possible at }\left(u_{t}^{*}, \alpha_{t}^{*}\right)
\end{array}\right.
$$

with

$$
\mathrm{L}_{\mathrm{b}}(t)=\sqrt{\frac{2 \pi^{2} \mathrm{~W}_{1}}{\mathrm{~S}_{t}^{\prime \prime} \Sigma_{t}^{2}}} \mathrm{~L}_{t} .
$$

Remark 6. Since $\mathcal{D}_{+} \subset H^{1}(0, L)$, then $\min _{H^{1}(0, L)} \hat{\mathcal{R}}_{t} \leq \min _{\mathcal{D}_{+}} \hat{\mathcal{R}}_{t}$ and $\mathrm{L}_{\mathrm{b}}(t) \leq \mathrm{L}_{\mathbf{s}}(t)$. That means that a bifurcation is possible even though the homogeneous state is stable. Let us note that the ratio $\mathrm{L}_{\mathrm{s}}(t) / \mathrm{L}_{\mathrm{b}}(t)$ depends only on the function state $\alpha \mapsto \mathrm{E}(\alpha)$ and the parameter $\mathrm{w}_{1}$, but not on $\alpha \mapsto \mathrm{L}(\alpha)$. Indeed, comparing (44) to (67) gives

$$
\frac{\mathrm{L}_{\mathrm{b}}(t)}{\mathrm{L}_{\mathrm{s}}(t)}=\frac{\mathrm{S}_{t}^{\prime \prime}}{2 \mathrm{E}_{t} \mathrm{~S}_{t}^{\prime 2}}<1
$$

In the case of strongly brittle materials of Example 1, we have $\frac{\mathrm{L}_{\mathrm{b}}(t)}{\mathrm{L}_{\mathrm{s}}(t)}=\frac{q+1}{2 q}$ with $q>1$. In the case of weakly brittle materials with softening $(q>1)$ of Example 2, we have $\frac{\mathrm{L}_{\mathrm{b}}(t)}{\mathrm{L}_{\mathrm{s}}(t)}=\frac{q-1}{2 q}$. Thus, in both cases, this ratio is a constant independent of the state and only dependent on the parameter q, see Figure 3.

\subsection{The damage modes of bifurcation}

At this stage, we have only proved that another solution than the homogeneous rate could exist for the bifurcation problem (61) when $\mathcal{A}_{t}$ is not definite positive. We have now to prove that another solution really exists in such a case and to determine it (or them).

After the change of variable $x \mapsto x / L$ and introducing the three dimensionless parameters

$$
\mathrm{a}=\frac{2 \mathrm{w}_{1} \mathrm{~L}_{t}^{2}}{\mathrm{~S}_{t}^{\prime \prime} \Sigma_{t}^{2} L^{2}}, \quad \mathrm{~b}=\frac{2 \mathrm{E}_{t}}{\mathrm{~S}_{t}^{\prime \prime} \Sigma_{t}^{2}}, \quad \mathrm{c}=\mathrm{S}_{t}^{\prime} \Sigma_{t}
$$



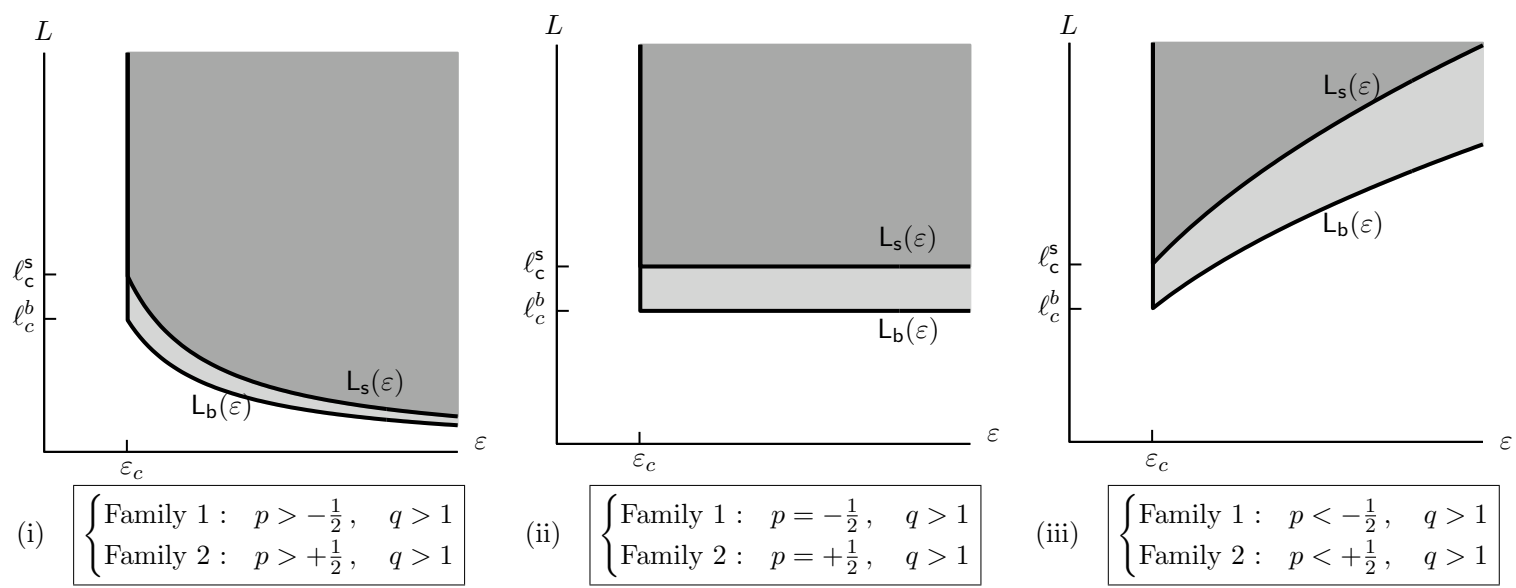

Figure 3: Domains of stable without bifurcation (white area), stable with bifurcation (light gray area) and unstable (dark gray area) homogeneous states in a diagram $L-\varepsilon(L=$ length of the bar, $\varepsilon=$ homogeneous strain) for a given $q$ and different values of $p$ for the families of strongly brittle materials of Example 1 or weakly brittle materials with softening of Example 2.

the variational problem (61) becomes (B.1), see Appendix B. We can only consider the case when the homogeneous state is stable but a bifurcation is possible, i.e. when $\min _{H^{1}(0,1)} \hat{\mathcal{R}}_{t} \leq$ $1<\min _{\mathcal{D}_{+}} \hat{\mathcal{R}}_{t}$ which is equivalent to $1<\pi \sqrt{\mathrm{a}} \mathrm{bc}^{2} \leq \mathrm{bc}^{2}$ and hence to

$$
\mathrm{L}_{\mathrm{b}}(t) \leq L<\mathrm{L}_{\mathrm{s}}(t) \text {. }
$$

In this situation, it is proved in Appendix B that the bifurcation problem admits other solutions than the homogeneous rate. In particular the damage rate $\dot{\alpha}$ which consists in a half-sinusoid whose support is $\left[0, \mathrm{~L}_{\mathrm{b}}(t)\right)$,

$$
\dot{\alpha}(x)= \begin{cases}\frac{\mathrm{L}_{\mathrm{s}}(t) L}{\mathrm{~L}_{\mathrm{b}}(t)\left(\mathrm{L}_{\mathrm{s}}(t)-L\right) \mathrm{S}_{t}^{\prime} \Sigma_{t}}\left(1+\cos \frac{\pi x}{\mathrm{~L}_{\mathrm{b}}(t)}\right) & \text { in }\left(0, \mathrm{~L}_{\mathrm{b}}(t)\right) \\ 0 & \text { otherwise }\end{cases}
$$

is solution, as well as its symmetric $\dot{\alpha}_{*}(x)=\dot{\alpha}(L-x)$. The associated strain rate field is given by

$$
\dot{\varepsilon}(x)=-\frac{\mathrm{L}_{\mathrm{s}}(t)}{\mathrm{L}_{\mathrm{s}}(t)-L}+\left\{\begin{array}{ll}
\frac{\mathrm{L}_{\mathrm{s}}(t) L}{\mathrm{~L}_{\mathrm{b}}(t)\left(\mathrm{L}_{\mathrm{s}}(t)-L\right)}\left(1+\cos \frac{\pi x}{\mathrm{~L}_{\mathrm{b}}(t)}\right) & \text { in }\left(0, \mathrm{~L}_{\mathrm{b}}(t)\right) \\
0 & \text { otherwise }
\end{array} .\right.
$$

Other solutions exist if $\mathrm{L}_{\mathrm{s}}(t) \geq L \geq 2 \mathrm{~L}_{\mathrm{b}}(t)$. All are made of identical sinusoids of half-length $\mathrm{L}_{\mathrm{b}}(t)$. The maximal number of half-sinusoids is equal to $L / \mathrm{L}_{\mathbf{b}}(t)$.

Example 7. In the case of the family of strongly brittle models of Example 1, then $\mathrm{bc}^{2}=$ $2 q /(q+1)$ with $q>1$ and the condition (69) gives

$$
\frac{L}{2}<\frac{q+1}{2 q} L<\mathrm{L}_{\mathrm{b}}(t) \leq L<\mathrm{L}_{\mathrm{s}}(t)
$$


Therefore, when the length $L$ of the bar is in the interval $\left(\mathrm{L}_{\mathrm{b}}(t), \mathrm{L}_{\mathbf{s}}(t)\right)$, there exists exactly two modes of bifurcation, the half-sinusoid $\dot{\alpha}$ and its symmetric $\dot{\alpha}_{*}$, because it is impossible to put more than one half-sinusoid of length $\mathrm{L}_{\mathrm{b}}(t)$ inside the bar.

Example 8. In the case of the family of weakly brittle models with softening of Example 2, then $\mathrm{bc}^{2}=2 q /(q-1)$ with $q>1$ and the condition (69) gives

$$
\frac{q-1}{2 q} L<\mathrm{L}_{\mathrm{b}}(t) \leq L<\mathrm{L}_{\mathrm{s}}(t) .
$$

Therefore, when the length $L$ of the bar is in the interval $\left(\mathrm{L}_{\mathrm{b}}(t), \mathrm{L}_{\mathrm{s}}(t)\right)$, there exist several modes of bifurcation, their number depending on $q$. For instance, when $q=2$ and hence $(q-1) / 2 q=1 / 4$, it is possible to construct modes of bifurcation which contains from one to three half-sinusoids when $L=3 \mathrm{~L}_{\mathrm{b}}(t)$.

Remark 7. Our proof of existence of branches of solution of the evolution problem which bifurcate from a homogeneous state is not complete, because we have merely proved that there exist non constant evolution rates solutions of the bifurcation problem. A complete proof should require to construct solutions of the evolution problem in a time interval $[t, t+\eta)$ with $\eta>0$. This proof is outside the scope of our paper, but the interested reader can refer to Benallal and Marigo (2007) or Pham and Marigo (2009a). In Benallal and Marigo (2007) such explicit constructions are made in the particular case of the weakly brittle model with softening of Example 2 whith $q=2$ and $p=0$, whereas in Pham and Marigo (2009a) a general method of construction of damage localized solutions is proposed and discussed for a broader class of softening laws.

\subsection{Stability of the bifurcated branches}

The bifurcated branches are experimentally observable only if they correspond to stable states. The following proposition gives an important result on the stability of the branches bifurcating from a stable homogeneous state.

Proposition 4.5. Let $\left(u_{t}^{*}, \alpha_{t}^{*}\right)$ be a homogeneous state of a bar of length $L<\mathrm{L}_{\mathbf{s}}(t)$. Let $\tau \mapsto$ $\left(u_{\tau}, \alpha_{\tau}\right)$ be a continuous evolution in the interval $[t, t+\eta)$ which starts from $\left(u_{t}^{*}, \alpha_{t}^{*}\right)$ at time $t$ and which satisfies (st) in the interval $(t, t+\eta)$. Then, for $\eta$ sufficiently small, all the states of this branch are stable, i.e. satisfy (ST).

Proof. We denote by $\|\cdot\|$ the natural norm on $H^{1}(0, L)$. Let $\tau \in(t, t+\eta),(v, \beta) \in \mathcal{C}^{0} \times \mathcal{D}_{+}$, $(v, \beta) \neq(0,0)$, and let $h$ be a small positive real number. Expanding $\mathcal{E}\left(u_{\tau}+h v, \alpha_{\tau}+h \beta\right)$ with respect to $h$ up to the second order gives

$$
\mathcal{E}\left(u_{\tau}+h v, \alpha_{\tau}+h \beta\right)=\mathcal{E}\left(u_{\tau}, \alpha_{\tau}\right)+h \mathcal{E}^{\prime}\left(u_{\tau}, \alpha_{\tau}\right)(v, \beta)+\frac{h^{2}}{2} \mathcal{E}^{\prime \prime}\left(u_{\tau}, \alpha_{\tau}\right)(v, \beta)+o\left(h^{2}\right) .
$$


Since the evolution satisfies (st), we have $\mathcal{E}^{\prime}\left(u_{\tau}, \alpha_{\tau}\right)(v, \beta) \geq 0$. Thus, it is sufficient to prove that $\mathcal{E}^{\prime \prime}\left(u_{\tau}, \alpha_{\tau}\right)(v, \beta)>0$ for proving the stability of $\left(u_{\tau}, \alpha_{\tau}\right)$ in the direction $(v, \beta)$. By continuity, the quadratic form $\mathcal{E}^{\prime \prime}\left(u_{\tau}, \alpha_{\tau}\right)$ converges to the quadratic form $\mathcal{E}^{\prime \prime}\left(u_{t}^{*}, \alpha_{t}^{*}\right)$ when $\tau$ tends to $t$ and

$$
\forall(v, \beta) \in \mathcal{C}^{0} \times H^{1}(0, L), \quad\left|\left(\mathcal{E}^{\prime \prime}\left(u_{\tau}, \alpha_{\tau}\right)-\mathcal{E}^{\prime \prime}\left(u_{t}^{*}, \alpha_{t}^{*}\right)\right)(v, \beta)\right| \leq O(\tau-t)\left(\|v\|^{2}+\|\beta\|^{2}\right)
$$

where $O(\cdot)$ is bounded on $[0, \eta)$ and $\lim _{s \rightarrow 0} O(s)=0$. Therefore, it is sufficient to prove that there exists $\mathrm{k}_{t}>0$ such that

$$
\forall(v, \beta) \in \mathcal{C}^{0} \times \mathcal{D}_{+}, \quad \mathcal{E}^{\prime \prime}\left(u_{t}^{*}, \alpha_{t}^{*}\right)(v, \beta) \geq \mathrm{k}_{t}\left(\|v\|^{2}+\|\beta\|^{2}\right) .
$$

Indeed, in such a case, for $\eta$ sufficiently small, we will have for all $\tau \in[t, t+\eta)$

$$
\mathcal{E}^{\prime \prime}\left(u_{\tau}, \alpha_{\tau}\right)(v, \beta) \geq\left(\mathrm{k}_{t}-O(\tau-t)\right)\left(\|v\|^{2}+\|\beta\|^{2}\right)>0 .
$$

Since $L<\mathrm{L}_{\mathrm{s}}(t)$, the state $\left(u_{t}^{*}, \alpha_{t}^{*}\right)$ is stable and $\mathrm{R}_{t}=\min _{\mathcal{C}^{0} \times \mathcal{D}_{+}} \mathcal{R}_{t}>1$. By definition of $\mathcal{R}_{t}$, see (41), we get for all $(v, \beta) \in \mathcal{C}^{0} \times \mathcal{D}_{+}$

$$
\mathcal{E}^{\prime \prime}\left(u_{t}^{*}, \alpha_{t}^{*}\right)(v, \beta) \geq\left(1-\frac{1}{\mathrm{R}_{t}}\right)\left(\mathrm{w}_{1} \mathrm{~L}_{t}^{2} \int_{0}^{L} \beta^{\prime 2} d x+\mathrm{E}_{t} \int_{0}^{L}\left(v^{\prime}-\mathrm{S}_{t}^{\prime} \Sigma_{t} \beta\right)^{2} d x\right) \geq 0
$$

with the equality to 0 if and only if $(v, \beta)=(0,0)$. Then, by standard arguments, we obtain (70).

This proposition proves that, when the length of the bar is such that $\mathrm{L}_{\mathrm{b}}(t) \leq L<\mathrm{L}_{\mathrm{s}}(t)$, then not only the homogeneous state $\left(u_{t}^{*}, \alpha_{t}^{*}\right)$ is stable, but also all the states sufficiently close to $\left(u_{t}^{*}, \alpha_{t}^{*}\right)$ and belonging to a bifurcated branch (or the homogeneous branch). From a practical viewpoint this result is rather bad, since it renders possible the bifurcation from the homogeneous branch to a bifurcated branch even if the homogeneous state is still stable. Moreover, it is not $a$ priori possible to know whether the bifurcation will really arise, because that might depend on dynamical effects or on the presence of imperfections which could favor one branch rather than the other.

\subsection{Interpretation in a stress-strain diagram}

Let us calculate the stress rate $\dot{\sigma}$ associated with a non constant solution of the bifurcation problem. Let $n$ be the number of half-sinusoids of this solution, $n$ must be such that

$$
\mathrm{L}_{\mathrm{b}}(t) \leq n \mathrm{~L}_{\mathrm{b}}(t) \leq L<\mathrm{L}_{\mathrm{s}}(t) .
$$

Then using (B.6) and (58) we get $\dot{\sigma}$ which can be compared to the stress rate $\dot{\Sigma}_{t}$ associated with the homogeneous solution. Specifically, we obtain

$$
\dot{\sigma}=-\frac{\mathrm{E}_{t} L}{n \mathrm{~L}_{\mathrm{s}}(t)-L}, \quad \dot{\Sigma}_{t}=-\frac{\mathrm{E}_{t} \mathrm{~L}_{\mathrm{b}}(t)}{\mathrm{L}_{\mathrm{s}}(t)-\mathrm{L}_{\mathrm{b}}(t)} .
$$


Since $L \geq n \mathrm{~L}_{\mathrm{b}}(t)$, we have $|\dot{\sigma}| \geq\left|\dot{\Sigma}_{t}\right|$ which means that the decrease of the stress associated with any mode of bifurcation is greater than that associated with the homogeneous evolution. In other words, the localization of damage increases the softening behavior of the bar.

When $L=\mathrm{L}_{\mathrm{b}}(t)$, then $n=1$ and $\dot{\sigma}=\dot{\Sigma}$. It is a limit case where the bifurcation branch is tangent to the homogeneous branch. The two branches have the same slope, but the evolution rates $(\dot{u}, \dot{\alpha})$ are different (that corresponds in Appendix B to the case 1 where $\pi \sqrt{\mathrm{a}}=1, n=1$ ).

When $n=1$ and $L$ tends to $\mathrm{L}_{\mathrm{s}}(t)$, then $\dot{\sigma}$ tends to infinity. It is a limit case where the bifurcation branch has a vertical slope (limit of a snap-back), the amplitude of the damage rate being infinite.

Example 9. In the case of strongly brittle materials of family 1 with $p>-\frac{1}{2}$ and $q>1$, the function $\varepsilon \mapsto \mathrm{L}_{\mathrm{b}}(\varepsilon)$ is decreasing. For a bar with length $L \leq \ell_{c}^{b}=(q+1) \ell_{\mathrm{c}}^{\mathrm{s}} / 2 q$, we will obtain the following responses during a monotonic loading where $\varepsilon$ increases, see Figure $4(i)$ :

1. The response follows the homogeneous branch as long as $\varepsilon<\varepsilon_{\mathbf{b}}(L)=\left(L / \ell_{c}^{b}\right)^{\frac{1-q}{2 p+1}} \varepsilon_{c}$;

2. When $\varepsilon$ is the interval $\left[\varepsilon_{\mathbf{b}}(L), \varepsilon_{\mathbf{s}}(L)\right], \varepsilon_{\mathbf{s}}(L)=\left(L / \ell_{\mathrm{c}}^{\mathrm{s}}\right)^{\frac{1-q}{2 p+1}} \varepsilon_{\mathrm{c}}$, the response can bifurcate from any homogeneous point to the unique possible bifurcated branch passing through this point. The bifurcation is tangent if that happens at $\varepsilon_{\mathbf{b}}(L)$ while the bifurcated branch is vertical if that happens at $\varepsilon_{\mathbf{s}}(L)$.

3. When $\varepsilon>\varepsilon_{\mathbf{s}}(L)$, the homogeneous branch is no more stable and the evolution necessarily follows a bifurcated branch (or the bar is broken).

In the case of weakly brittle materials of family 2 with $p>\frac{1}{2}$ and $q>1$, the function $\varepsilon \mapsto \mathrm{L}_{\mathrm{b}}(\varepsilon)$ is decreasing. For a bar with length $L \leq \ell_{c}^{b}=(q-1) \ell_{\mathrm{c}}^{\mathrm{s}} / 2 q$, we will obtain the following responses, see Figure 4(ii):

1. The response follows the homogeneous branch as long as $\varepsilon<\varepsilon_{\mathbf{b}}(L)=\left(L / \ell_{c}^{b}\right)^{\frac{q+1}{1-2 p}} \varepsilon_{c}$;

2. When $\varepsilon$ is in the interval $\left[\varepsilon_{\mathbf{b}}(L), \varepsilon_{\mathbf{s}}(L)\right], \varepsilon_{\mathbf{s}}(L)=\left(L / \ell_{\mathbf{c}}^{\mathbf{s}}\right)^{\frac{q+1}{1-2 p}} \varepsilon_{\mathbf{c}}$, the response can bifurcate to one of the possible bifurcated branches passing through a homogeneous point. The bifurcation is necessarily tangent if that happens at $\varepsilon_{\mathrm{b}}(L)$ while the bifurcated branch can be vertical if that happens at $\varepsilon_{\mathbf{s}}(L)$.

3. When $\varepsilon>\varepsilon_{\mathbf{s}}(L)$, the homogeneous branch is no more stable and the evolution necessarily follows a bifurcated branch (or the bar is broken).

\section{Some consequences on the identification of the model from uniaxial tests}

The goal of this section is to propose a procedure to identify the two constitutive parameters $\alpha_{m}, \mathrm{w}_{1}$ and the two constitutive functions $\alpha \mapsto \mathrm{E}(\alpha), \alpha \mapsto \mathrm{L}(\alpha)$ with the only use of uniaxial 


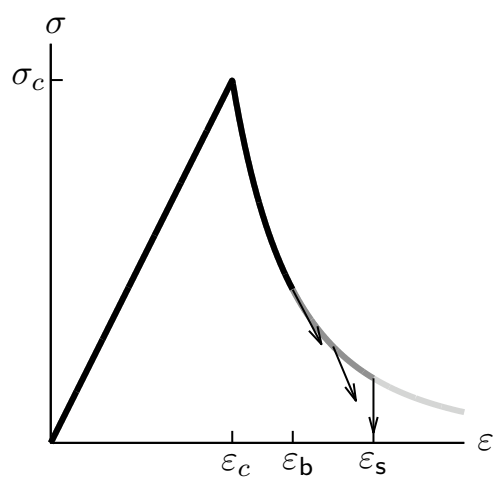

(i) strongly brittle

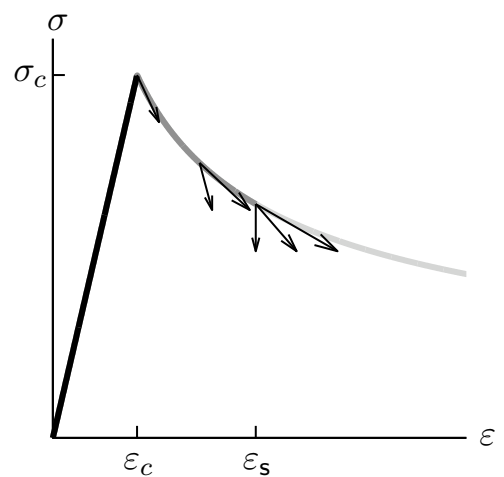

(ii) weakly brittle

Figure 4: Different possibilities of bifurcation from the homogeneous response. The curves represent the homogeneous response (in black: the stable states without possible bifurcation; in dark gray: the stable states with possible bifurcation; in light gray: the unstable states). The arrows represent the different possible directions of bifurcation at three particular points (the first is at $\varepsilon_{\mathbf{b}}(L)$; the third is at $\varepsilon_{\mathbf{s}}(L)$ ). (i) The model of family 1 with $p=0$ and $q=2, L=3 \ell_{c}^{b} / 4$; (ii) The model of family 2 with $p=3$ and $q=3, L=\ell_{c}^{b}$; note that $\varepsilon_{\mathrm{b}}(L)=\varepsilon_{c}$.

tests. We will not discuss the practical feasibility of the procedure, but we assume that it is possible to detect at each time whether the strain field is homogeneous in space.

\subsection{Identification of $\alpha_{m}, \mathrm{w}_{1}$ and $\alpha \mapsto \mathrm{E}(\alpha)$}

For a bar of given length, one measures for each value of the overall stretching $\varepsilon$ in a given interval $\left[0, \varepsilon_{M}\right]$ the stress $\sigma$ and check whether the strain field is homogeneous. If not, one decreases the length of the bar, makes the uniaxial test again and repeats the procedure until the response becomes homogeneous in the full range of $\varepsilon$. (Theoretically, there exists a threshold $L_{m}$, which can depend on $\varepsilon_{M}$, for the length of the bar under which no bifurcation from the homogeneous response is possible. Indeed, by continuity arguments, $L_{m}=\min _{\varepsilon \in\left[\varepsilon_{c}, \varepsilon_{M}\right]} L_{b}(\varepsilon)$.)

From these measures of $\varepsilon$ and $\sigma$, one proceeds as follows:

1. One determines the sound Young modulus $\mathrm{E}_{0}$;

2. One determines the critical strain $\varepsilon_{\mathrm{c}}$ (and hence the critical stress $\sigma_{\mathrm{c}}$ ) as the value of the strain after which the response is no more linear;

3. One obtains the function $\mathrm{F}$ from the relation $\sigma=\mathrm{F}(\varepsilon)$ when $\varepsilon \in\left[\varepsilon_{c}, \varepsilon_{M}\right]$;

4. By integration (which can be made graphically), one obtains the damage dissipated energy $\mathrm{w}(\varepsilon)$ for each $\varepsilon$, see Section 3.2 anf Figure 5.

5. For discriminating if $\alpha_{m}$ is finite or infinite, one should make the test for all $\varepsilon$ (i.e. take $\left.\varepsilon_{M}=+\infty\right)$. In practise, one can extrapolate the function $F$ for large values of $\varepsilon$, for instance by fitting by the best power law, and then conclude. If $\mathrm{w}(\varepsilon)$ tends to a finite limit when $\varepsilon$ goes to infinity, one sets $\mathrm{w}_{1}=\lim _{\varepsilon \rightarrow \infty} \mathrm{w}(\varepsilon)$. Otherwise, one sets $\alpha_{m}=+\infty$, chooses arbitrarily 
a certain $\varepsilon_{1}$ (for instance $\varepsilon_{1}=2 \varepsilon_{\mathrm{c}}$ ) and takes for $\mathrm{w}_{1}$ the dissipated energy at this $\varepsilon_{1}$, i.e. $\mathrm{w}_{1}=\mathrm{w}\left(\varepsilon_{1}\right)$.

6. One sets $\alpha=\mathrm{w}(\varepsilon) / \mathrm{w}_{1}$ and one inverts the relation to obtain $\varepsilon=\mathrm{e}(\alpha)$.

7. One obtains $\mathrm{E}(\alpha)$ by $\mathrm{E}(\alpha)=\mathrm{F}(\mathrm{e}(\alpha)) / \mathrm{e}(\alpha)$ and then $\mathrm{S}(\alpha)=1 / \mathrm{E}(\alpha)$.

8. One obtains the first derivatives with the same accuracy than $\mathrm{E}(\alpha)$ by virtue of the relations $-\mathrm{E}^{\prime}(\alpha)=2 \mathrm{w}_{1} / \mathrm{e}(\alpha)^{2}$ and $\mathrm{S}^{\prime}(\alpha)=-\mathrm{E}^{\prime}(\alpha) \mathrm{S}(\alpha)^{2}$.

9. One obtains the second derivatives with a good accuracy provided that the slope $\mathrm{F}^{\prime}(\varepsilon)$ of the stress-strain curve is itself measured with a good accuracy. Indeed, one can use for instance the following relation for $\mathrm{S}^{\prime \prime}(\alpha)$ :

$$
\mathrm{S}^{\prime \prime}(\alpha)=-\frac{8 \mathrm{w}_{1}^{2} \mathrm{~F}^{\prime}(\varepsilon)}{\mathrm{F}(\varepsilon)^{3}\left(\mathrm{~F}(\varepsilon)-\mathrm{F}^{\prime}(\varepsilon) \varepsilon\right)} \quad \text { with } \quad \varepsilon=\mathrm{e}(\alpha)
$$

Proof. By definition $\mathrm{w}(\varepsilon)=\int_{0}^{\varepsilon} \mathrm{F}(e) d e-\frac{1}{2} \mathrm{~F}(\varepsilon) \varepsilon$ and hence $2 \mathrm{w}^{\prime}(\varepsilon)=\mathrm{F}(\varepsilon)-\mathrm{F}^{\prime}(\varepsilon) \varepsilon$. By construction, $\mathrm{w}_{1} \alpha=\mathrm{w}(\varepsilon)$ and hence $2 \mathrm{w}_{1} \frac{d \alpha}{d \varepsilon}=\mathrm{F}(\varepsilon)-\mathrm{F}^{\prime}(\varepsilon) \varepsilon$. Then, differentiating the equality $\mathrm{S}^{\prime}(\alpha) \mathrm{F}(\varepsilon)^{2}=2 \mathrm{w}_{1}$ with respect to $\varepsilon$ leads to $0=\mathrm{S}^{\prime \prime}(\alpha) \frac{d \alpha}{d \varepsilon} \mathrm{F}(\varepsilon)^{2}+2 \mathrm{~S}^{\prime}(\alpha) \mathrm{F}^{\prime}(\varepsilon) \mathrm{F}(\varepsilon)$. After easy calculations and using the definition $\varepsilon=\mathrm{e}(\alpha)$, we get (72).
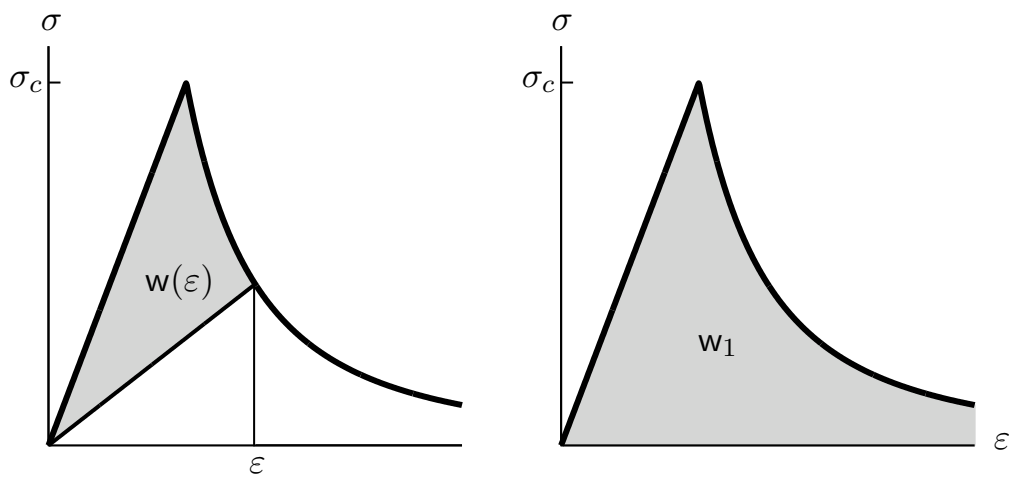

Figure 5: Measurement of the damage dissipated energy during a homogeneous evolution, using the stress-strain response. Left: definition of $\mathrm{w}(\varepsilon)$; Right: definition of $\mathrm{w}_{1}=\mathrm{w}(+\infty)$ when $\mathrm{w}(+\infty)<\infty$.

\subsection{Identification of $\alpha \mapsto \mathrm{L}(\alpha)$}

The function $\alpha \mapsto \mathrm{L}(\alpha)$ does not influence the shape of the stress-strain curve $\sigma=\mathrm{F}(\varepsilon)$, but is essential for assessing its stability. Since the homogeneous response is always stable without any possible bifurcation in the case of a hardening behavior (i.e. when $\mathrm{F}$ is increasing), it is impossible to identify $\alpha \mapsto \mathrm{L}(\alpha)$ with uniaxial tests alone for such materials. Accordingly, we only consider behavior with softening, i.e. $\mathrm{F}$ is assumed to be decreasing. 
Let us first remark that if one is able to measure $L_{s}(\varepsilon)$, i.e. the length of the bar beyond which the homogeneous state $\left(u^{*}=\varepsilon x, \alpha^{*}=\mathrm{w}(\varepsilon) / \mathrm{w}_{1}\right)$ is no more stable, then one obtains $\mathrm{L}\left(\alpha^{*}\right)$. Indeed, by virtue of (44), we have

$$
\mathrm{L}\left(\alpha^{*}\right)=\sqrt{\frac{\mathrm{S}^{\prime \prime}\left(\alpha^{*}\right)^{3} \varepsilon^{2}}{8 \pi^{2} \mathrm{~S}^{\prime}\left(\alpha^{*}\right)^{4} \mathrm{~W}_{1}}} \mathrm{~L}_{\mathbf{s}}(\varepsilon)
$$

where $\mathrm{S}^{\prime}\left(\alpha^{*}\right)$ and $\mathrm{S}^{\prime \prime}\left(\alpha^{*}\right)$ can be obtained from the stress-strain curve. However, it is not always possible for measuring $L_{s}(\varepsilon)$ with monotonically increasing uniaxial tests. Indeed, let us consider a material whose stability function $\varepsilon \mapsto \mathrm{L}_{\mathrm{s}}(\varepsilon)$ is increasing like in Figure 6 or Figure 3(iii). If

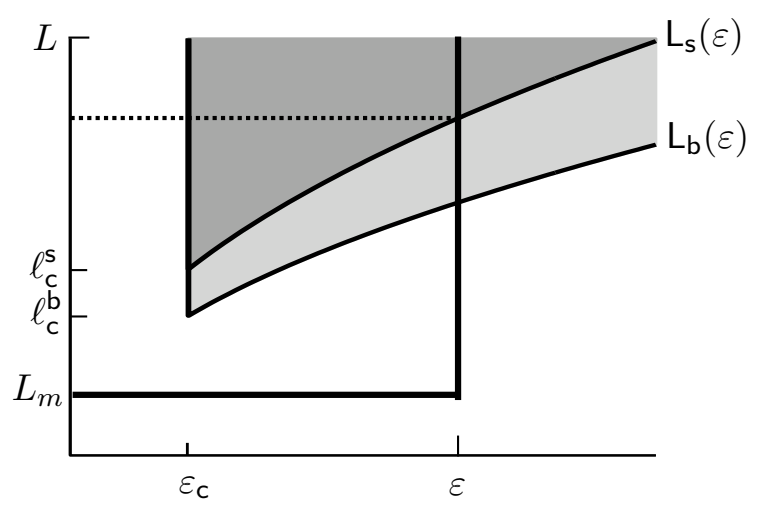

Figure 6: Path of loading for measuring $L_{b}(\varepsilon)$. First stage: the length of the bar is fixed at $L_{m}$ and the overall strain is increasing from 0 to $\varepsilon$; second stage: the overall strain is fixed at $\varepsilon$ and the length of the bar is increasing up to $L$.

the length of the bar is small (less than $\ell_{c}^{b}$ ), then the homogeneous response is stable and no bifurcation is possible whatever the overall strain. We cannot obtain any information about $\mathrm{L}\left(\alpha^{*}\right)$. If the length of the bar is too large (greater than $\ell_{\mathrm{c}}^{\mathrm{s}}$ ), then the homogeneous response is no more stable as soon as the critical strain is reached and we can obtain an information about $\mathrm{L}\left(\alpha^{*}\right)$ only by considering non homogeneous responses. Therefore, it is more convenient to consider another procedure which is able to give $L_{s}(\varepsilon)$ in any circumstance. The principle of this procedure is as follows:

1. One chooses a bar with a length $L$ sufficiently large so that $L>L_{s}(\varepsilon)$. (That requires to have a priori estimates on $\mathrm{L}_{\mathbf{s}}(\varepsilon)$ or to iterate the procedure.)

2. One equips the bar with a series of $n+1$ hard devices regularly spaced. The number of devices must be sufficiently large to ensure that their distance $L_{m}=L / n$ be sufficiently small so that the homogeneous response of a bar of length $L_{m}$ be stable without any bifurcation for all overall strains up to the desired value $\varepsilon$.

3. One increases progressively the displacement of each device so that the strain be homogeneous in the whole bar. Namely, the displacement $u^{i}(t)$ of the $i$-th device at time $t$ must be equal to $t i L_{m}, 0 \leq i \leq n$. Accordingly, at $t=\varepsilon$ the bar should be in the homogeneous state $\left(u^{*}, \alpha^{*}\right)$. 
4. Then, one removes the hard devices one by one, starting from one end and going to the other end, see Figure 7. One notes when the strain field in the "released" part of the bar is no more homogeneous. As it is proved below, one obtains that $L_{s}(\varepsilon) \in\left[i L_{m},(i+1) L_{m}\right)$ if the loss of homogeneity happens after the $i$-th device was removed.
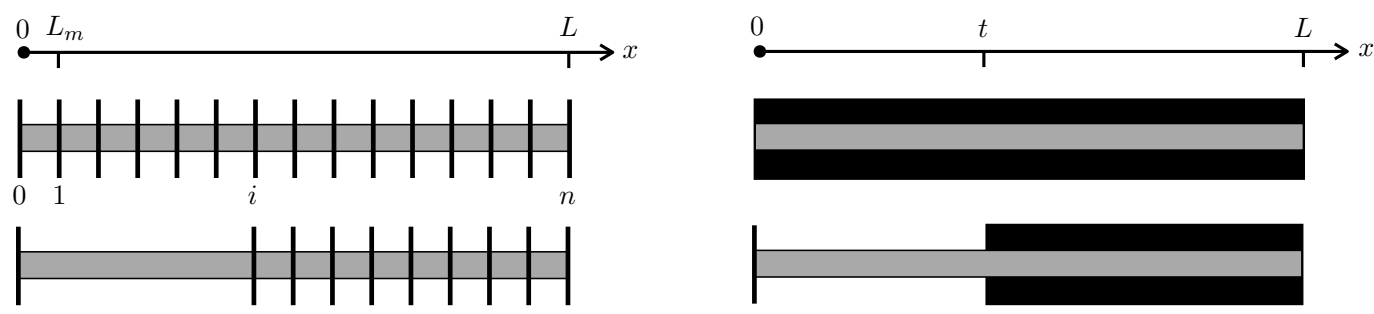

Figure 7: Procedure for measuring $\mathrm{L}_{b}(\varepsilon)$. Left: the practical apparatus where a series of equally spaced hard devices are progressively removed; Right: the theoretical version of this apparatus where the length $t$ of the unconstrained part of the bar grows continuously.

The proof that the homogeneous state is the only one that we will observe as long as the length of the released part of the bar is less than $L_{s}(\varepsilon)$ is made difficult because of the discrete character of the process. (In fact, the setting of the evolution problem itself is not easy, because we have to imagine a process during which the kinematical constraint is continuously released in time.) So, we replace the discrete apparatus above by a continuous one. Specifically, we starts from the initial condition where the state of the whole bar $(0, L)$ is the homogeneous one $\left(u^{*}, \alpha^{*}\right)$ and where the displacement of all the points of the bar is controlled. Then, we release the kinematical constraint in the part $(0, t)$ of the bar with $t$ increasing from 0 to $L$, see Figure 7 .

This procedure leads to an evolution problem similar to the general one formulated in Section 2.2. The length $t$ of the unconstrained part of the bar plays the role of the time. The unique change concerns the sets of kinematically admissible displacements. In the present case, we have

$$
\mathcal{C}_{t}=\left\{v \in H^{1}(0, L): v(0)=0, v(x)=\varepsilon x \text { if } x \in[t, L]\right\} .
$$

Hence, $\mathcal{C}_{t}$ is still an affine space, but the associated linear space is now time dependent and is denoted $\mathcal{C}_{t}^{0}$,

$$
\mathcal{C}_{t}^{0}=\left\{v \in H^{1}(0, L): v(0)=0, v(x)=0 \text { if } x \in[t, L]\right\} .
$$

Let us note that $\mathcal{C}_{t}^{0}$ is included in $\mathcal{C}^{0}=H_{0}^{1}(0, L)$ for all $t \in[0, L]$.

Accordingly, the evolution $\left(u_{t}, \alpha_{t}\right)$ of the bar must still satisfy (IR), (ST) and (EB), but with the initial condition $\left(u_{0}, \alpha_{0}\right)=\left(u^{*}, \alpha^{*}\right)$, with the new definition of $\mathcal{C}_{t}$ and with $\mathcal{C}_{t}^{0}$ instead of $\mathcal{C}^{0}$. Moreover, since $U_{t}=\varepsilon L$ for all $t$, we have $\dot{U}_{t}=0$ and hence (EB) simply reads as

$$
\mathcal{E}\left(u_{t}, \alpha_{t}\right)=\mathcal{E}\left(u^{*}, \alpha^{*}\right), \quad \forall t \in[0, L] .
$$

Thus, the total energy remains constant throughout the process. 
Notation 2. In the remaining part of this section we will use the simplified notations

$$
\mathrm{E}_{*}=\mathrm{E}\left(\alpha^{*}\right), \quad \mathrm{E}_{*}^{\prime \prime}=\mathrm{E}^{\prime \prime}\left(\alpha^{*}\right), \quad \mathrm{S}_{*}^{\prime}=\mathrm{S}^{\prime}\left(\alpha^{*}\right), \quad \mathrm{S}_{*}^{\prime \prime}=\mathrm{S}^{\prime \prime}\left(\alpha^{*}\right), \quad \Sigma_{*}=\mathrm{E}\left(\alpha^{*}\right) \varepsilon, \quad \mathrm{L}_{*}=\mathrm{L}\left(\alpha^{*}\right) .
$$

Moreover, we will frequently refer to the analysis or the results of the evolution problem associated with the monotonically increasing uniaxial tension test of Section 2.2. In such a case, it is necessary to use the following change of notations where the original parameters are those relative to the evolution problem of Section 2.2 and the final ones are those of the current evolution problem:

$$
t \mapsto \varepsilon, \quad L \mapsto t, \quad \alpha_{t}^{*} \mapsto \alpha^{*}
$$

Let us first prove the following expected result

Proposition 5.1. The evolution consisting in the constant homogeneous state, i.e. $\left(u_{t}, \alpha_{t}\right)=$ $\left(u^{*}, \alpha^{*}\right)$ for $t \in[0, L]$, is solution of the evolution problem as long as $t<\mathrm{L}_{\mathrm{s}}(\varepsilon)$, but this state is no more stable as soon as $t>\mathrm{L}_{\mathrm{s}}(\varepsilon)$.

Proof. (IR) and (EB) are automatically satisfied at all $t$. It remains to determine when the state is stable. Let $t \in[0, L], h>0$ and $(v, \beta) \in \mathcal{C}_{t}^{0} \times \mathcal{D}_{+}$. Expanding $\mathcal{E}\left(u^{*}+h v, \alpha^{*}+h \beta\right)$ up to the second order in $h$ leads to

$$
\mathcal{E}\left(u^{*}+h v, \alpha^{*}+h \beta\right)-\mathcal{E}\left(u^{*}, \alpha^{*}\right)=\frac{h^{2}}{2} \mathcal{E}^{\prime \prime}\left(u^{*}, \alpha^{*}\right)(v, \beta)+o\left(h^{2}\right),
$$

because we have still $\mathcal{E}^{\prime}\left(u^{*}, \alpha^{*}\right)(v, \beta)=0$. (Indeed, since $\mathcal{C}_{t}^{0} \subset \mathcal{C}^{0}$, the equality (39) with $\varepsilon$ instead of $t$ holds true.) Therefore, the stability of the state $\left(u^{*}, \alpha^{*}\right)$ depends on the sign of the second derivative which reads here as

$$
\begin{aligned}
\mathcal{E}^{\prime \prime}\left(u^{*}, \alpha^{*}\right)(v, \beta)= & \int_{0}^{t}\left(\mathrm{w}_{1} \mathrm{~L}_{*}^{2} \beta^{\prime}(x)^{2}+\mathrm{E}_{*}\left(v^{\prime}(x)-\mathrm{S}_{*}^{\prime} \Sigma_{*} \beta(x)\right)^{2}-\frac{1}{2} \mathrm{~S}_{*}^{\prime \prime} \Sigma_{*}^{2} \beta(x)^{2}\right) d x \\
& +\int_{t}^{L}\left(\mathrm{w}_{1} \mathrm{~L}_{*}^{2} \beta^{\prime}(x)^{2}+\frac{1}{2} \mathrm{E}_{*}^{\prime \prime} \varepsilon^{2} \beta(x)^{2}\right) d x .
\end{aligned}
$$

In $(77)$ the integral over $(t, L)$ is non negative and the integrals over $(0, t)$ are the same as in (40) (after the change of notations (76)). Accordingly, using the results of Section 3.3, we obtain that $\mathcal{E}^{\prime \prime}\left(u^{*}, \alpha^{*}\right)(v, \beta) \geq 0$ when $t<\mathrm{L}_{\mathrm{s}}(\varepsilon)$. Moreover, since the equality holds only when $(v, \beta)=(0,0)$, we can conclude that $\left(u^{*}, \alpha^{*}\right)$ is stable when $t<\mathrm{L}_{\mathbf{s}}(\varepsilon)$.

When $t>\mathrm{L}_{\mathbf{s}}(\varepsilon)$, let us choose $(v, \beta) \in \mathcal{C}_{t}^{0} \times \mathcal{D}_{+}$such that

$$
\beta(x)=\left\{\begin{array}{ll}
1+\cos \frac{\pi x}{\mathrm{~L}_{\mathrm{s}}(\varepsilon)}, & \text { if } x \in\left(0, \mathrm{~L}_{\mathrm{s}}(\varepsilon)\right) \\
0 & \text { otherwise }
\end{array},\right.
$$




$$
v(0)=0, \quad v^{\prime}(x)=\mathrm{S}_{*}^{\prime} \Sigma_{*} \cdot\left\{\begin{array}{ll}
\left(1-\mathrm{L}_{\mathbf{s}}(\varepsilon) / t+\cos \frac{\pi x}{\mathrm{~L}_{\mathbf{s}}(\varepsilon)}\right) & , \text { if } x \in\left(0, \mathrm{~L}_{\mathbf{s}}(\varepsilon)\right) \\
-\mathrm{L}_{\mathbf{s}}(\varepsilon) / t & , \text { if } x \in\left(\mathrm{L}_{\mathbf{s}}(\varepsilon), t\right) \\
0 & , \text { otherwise }
\end{array} .\right.
$$

After the change of notations (76), the restrictions of $(v, \beta)$ to $[0, t]$ is a minimizer of the Rayleigh ratio (41) over $\mathcal{C}_{t}^{0} \times \mathcal{D}_{+}$, see Appendix A.2. Since these fields lead to a Rayleigh ratio less than 1 , the integral over $(0, t)$ in $(77)$ is negative while the integral over $(t, L)$ vanishes. Therefore $\mathcal{E}^{\prime \prime}\left(u^{*}, \alpha^{*}\right)(v, \beta)<0$ and the state $\left(u^{*}, \alpha^{*}\right)$ is unstable.

Remark 8. The procedure which consists in removing the kinematical constraint progressively from one end of the bar is essential. Indeed, suppose to change the procedure by removing the constraints from the middle of the bar, i.e. in the growing interval $I_{t}=((L-t) / 2,(L+$ $t) / 2)$. If we choose for the restriction of $(v, \beta)$ to $I_{t}$ a minimizer of the Rayleigh ratio (41) over $H_{0}^{1}\left(I_{t}\right) \times\left\{\alpha \in H^{1}\left(I_{t}\right): \alpha \geq 0\right\}$, then $\beta$ does not vanish at one end of $I_{t}$, say for example $x=(L-t) / 2$. Hence, by continuity $\beta \neq 0$ in the still constrained part $(0,(L-t) / 2)$ and the corresponding integral in $\mathcal{E}^{\prime \prime}\left(u^{*}, \alpha^{*}\right)(v, \beta)$ is positive. Therefore, the stability result is changed and the homogeneous state remains stable until a value of $t$ greater than $\mathrm{L}_{\mathrm{s}}(\varepsilon)$. In other words, the constrained parts of the bar have a stabilizing effect on the unconstrained one. But, in our procedure where the constraints act only on one side, this stabilizing effect is not sufficient to change the critical value $\mathrm{L}_{\mathrm{s}}(\varepsilon)$.

The last step consists in proving the

Proposition 5.2. There exists no possibility of bifurcation from the homogeneous state $\left(u^{*}, \alpha^{*}\right)$ as long as $t<\mathrm{L}_{\mathrm{s}}(\varepsilon)$. At $t=\mathrm{L}_{\mathrm{s}}(\varepsilon)$ a bifurcation is possible in the direction $(\dot{u}, \dot{\alpha})$ given by

$$
\dot{\alpha}(x)=\left\{\begin{array}{ll}
A\left(1+\cos \frac{\pi x}{\mathrm{~L}_{\mathrm{s}}(\varepsilon)}\right) \\
0
\end{array}, \quad \dot{u}(x)= \begin{cases}A \mathrm{~S}_{*}^{\prime} \Sigma_{*} \frac{\mathrm{L}_{\mathrm{s}}(\varepsilon)}{\pi} \sin \frac{\pi x}{\mathrm{~L}_{\mathrm{s}}(\varepsilon)} & \text { if } x \in\left[0, \mathrm{~L}_{\mathrm{s}}(\varepsilon)\right] \\
0 & \text { otherwise }\end{cases}\right.
$$

where $A$ is an arbitrary dimensionless positive constant.

Proof. Let us first construct the rate problem giving the possible evolution rates $(\dot{u}, \dot{\alpha})$ at time $t$ associated with a solution of the evolution problem leaving the homogeneous state $\left(u^{*}, \alpha^{*}\right)$ at time $t$. This construction is quite similar to that of Section 4.1, we have only to change the definitions of $\mathcal{C}_{t}$ and $\mathcal{C}^{0}$ by (73) and (74), and to modify the energy balance according to (75). From (52) which is a consequence of (ST), we get $\dot{u}^{\prime}(x)=\mathrm{S}_{*}^{\prime} \Sigma_{*} \dot{\alpha}(x)+\dot{C}$ in $(0, t)$ where $\dot{C}$ is a constant, whereas $\dot{u}=0$ in $[t, L]$. The constant is given by the (new) conditions $\dot{u}(0)=\dot{u}(t)=0$ which leads to

$$
\dot{u}(x)=\mathrm{S}_{*}^{\prime} \Sigma_{*} \int_{0}^{x}(\dot{\alpha}(y)-\langle\dot{\alpha}\rangle) d y \quad \text { when } \quad x \in[0, t], \quad\langle\dot{\alpha}\rangle=\frac{1}{t} \int_{0}^{t} \dot{\alpha}(y) d y .
$$


Inserting (79) into (50) leads to the variational inequality for $\dot{\alpha}: \mathcal{A}_{t}^{*}(\dot{\alpha}, \beta) \geq 0, \forall \beta \in \mathcal{D}_{+}$, where $\mathcal{A}_{t}^{*}$ denotes the symmetric bilinear form defined on $H^{1}(0, L)^{2}$ by

$$
\begin{aligned}
\mathcal{A}_{t}^{*}(\dot{\alpha}, \beta)= & \mathrm{w}_{1} \mathrm{~L}_{*}^{2} \int_{0}^{t} \dot{\alpha}^{\prime}(x) \beta^{\prime}(x) d x+\mathrm{E}_{*} \mathrm{~S}_{*}^{\prime 2} \Sigma_{*}^{2} \frac{1}{t} \int_{0}^{t} \dot{\alpha}(x) d x \int_{0}^{t} \beta(x) d x-\frac{1}{2} \mathrm{~S}_{*}^{\prime \prime} \Sigma_{*}^{2} \int_{0}^{t} \dot{\alpha}(x) \beta(x) d x, \\
& +\int_{t}^{L}\left(\mathrm{w}_{1} \mathrm{~L}_{*}^{2} \dot{\alpha}^{\prime}(x) \beta^{\prime}(x)+\frac{1}{2} \mathrm{E}_{*}^{\prime \prime} \varepsilon^{2} \dot{\alpha}(x) \beta(x)\right) d x .
\end{aligned}
$$

Let us consider the energy balance (75). Let $h$ be a small positive number and let us set $\chi_{t+h}=\left(u_{t+h}, \alpha_{t+h}\right), \chi^{*}=\chi_{t}=\left(u^{*}, \alpha^{*}\right)$. Expanding $\mathcal{E}\left(\chi_{t+h}\right)-\mathcal{E}\left(\chi^{*}\right)$ up to the second order and inserting into $(75)$ give

$$
0=\mathcal{E}^{\prime}\left(\chi^{*}\right)\left(\chi_{t+h}-\chi^{*}\right)+\frac{1}{2} \mathcal{E}^{\prime \prime}\left(\chi^{*}\right)\left(\chi_{t+h}-\chi^{*}\right)+o\left(\left\|\chi_{t+h}-\chi^{*}\right\|^{2}\right) .
$$

Since $u_{t+h}-u^{*} \in \mathcal{C}_{t}^{0} \subset \mathcal{C}^{0}$ and $\alpha_{t+h}-\alpha^{*} \in \mathcal{D}_{+}$, we have $\mathcal{E}^{\prime}\left(\chi^{*}\right)\left(\chi_{t+h}-\chi^{*}\right)=0$ by virtue of (39). Therefore, dividing (81) by $h^{2}$ and passing to the limit when $h$ goes to 0 , we finally obtain that the evolution rate must satisfy $\mathcal{E}^{\prime \prime}\left(\chi^{*}\right)(\dot{u}, \dot{\alpha})=0$. Using (79) and inserting into (77), we get $\mathcal{A}_{t}^{*}(\dot{\alpha}, \dot{\alpha})=0$.

Therefore, $\dot{\alpha}$ must be a solution of the following variational problem:

$$
\dot{\alpha} \in \mathcal{D}_{+}, \quad \mathcal{A}_{t}^{*}(\dot{\alpha}, \beta-\dot{\alpha}) \geq 0 \quad \forall \beta \in \mathcal{D}_{+}
$$

and then $\dot{u}$ is given by $(79)$. Of course, $(\dot{u}, \dot{\alpha})=(0,0)$ is solution at all $t$. That corresponds to the trivial branch $\left(u_{t}, \alpha_{t}\right)=\left(u^{*}, \alpha^{*}\right)$ for all $t$. Let us examine whether another solution exists.

1. When $t<\mathrm{L}_{\mathrm{s}}(\varepsilon)$. Then, using the results of Section 3.3, we obtain $\mathcal{E}^{\prime \prime}\left(u^{*}, \alpha^{*}\right)(v, \beta) \geq 0$ for all $(v, \beta) \in \mathcal{C}_{t}^{0} \times \mathcal{D}_{+}$and the equality holds only when $(v, \beta)=(0,0)$. Hence $(0,0)$ is the unique solution of the rate problem, no bifurcation is possible.

2. When $t=\mathrm{L}_{\mathrm{s}}(\varepsilon)$. Then, $\mathcal{A}_{t}^{*}(\dot{\alpha}, \dot{\alpha})=0$ and hence $\mathcal{E}^{\prime \prime}\left(\chi^{*}\right)(\dot{u}, \dot{\alpha})=0$ are possible if and only if $(\dot{u}, \dot{\alpha})=(0,0)$ in $[t, L]$ and the restriction of $(\dot{u}, \dot{\alpha})$ to $[0, t]$ is a minimizer of the Rayleigh ratio (41) (with the change of notations (76)) over $H_{0}^{1}(0, t) \times\left\{\beta \in H^{1}(0, t): \beta \geq 0\right\}$. That corresponds to the limit case where the minimum of the Rayleigh ratio is equal to 1 . Then, using the result of Proposition A.2 (the case $\pi^{2} a=b c^{2}$ ), we get (78).

Since the state $\left(u^{*}, \alpha^{*}\right)$ is no more stable when $t>\mathrm{L}_{\mathrm{s}}(\varepsilon)$, one can expects that either the evolution will follow the bifurcated branch at $t=\mathrm{L}_{\mathrm{s}}(\varepsilon)$ if this branch is stable, or the evolution will jump to another branch or the bar will break at $t=\mathrm{L}_{\mathrm{s}}(\varepsilon)$. In any case, one should observe a sufficiently noticeable event to identify $L_{s}(\varepsilon)$.

\section{Conclusion and perspectives}

Starting from a class of gradient damage models whose construction and evolution law are based on a variational approach, we have illustrated the regularizing effect induced by the 
gradient terms. Specifically we have shown that the presence of a gradient of damage term stabilizes the homogeneous response in an uniaxial test provided that the size of the specimen is small enough. In turn, these size effects give the opportunity to identify the constitutive functions by measuring only the homogeneous response and its loss of stability. To prevent that the measurements are polluted by bifurcations to non homogeneous branches before the loss of stability of the homogeneous state, we propose a procedure which prohibit such bifurcations. The main advantage of this procedure is that requires to measure only global quantities and simply to detect a loss of homogeneity of the strain field. A possible limitation of this procedure could be the requirement of tests on slender and sufficiently short specimens, which can be a difficult task for materials whose characteristic length is too small. In such a case, there is no other alternative but to identify the constitutive functions from damage localized responses. That will need to extend the study presented in this work to localized solutions, including a full stability

and bifurcation analysis. This task will be the subject of future works in the prolongation of those already achieved, like Benallal and Marigo (2007), Pham and Marigo (2009b) and Pham et al. (2010).

Another important task will consist in extending all these results to a three-dimensional setting. There is no theoretical impossibility to do that and, even, a part of this extension has been already made, see Pham and Marigo (2010a,b). The construction of the model, the setting of the evolution problem and the determination of the homogeneous response are quite similar to those in 1D. But there exists a practical difficulty to calculate the critical size of a $3 \mathrm{D}$ specimen beyond which the homogeneous state is no more stable. That needs in general numerical computations. However such a study deserves to be made, because new geometric parameters appear and probably play a role. For instance, it should be interesting to analyze the influence of the slenderness of a specimen (the ratio of the radius and the length in the case of a cylindrical specimen for instance) on that critical size. That could give access to some informations on the constitutive parameters.

\section{Acknowledgements}

The present work has been developed in the framework of the French research network MoMaS (Modélisations Mathématiques et Simulations numériques liées aux problèmes de gestion des déchets nucléaires). Its financial support is gratefully acknowledged.

\section{References}

Amor, H., Marigo, J.-J., Maurini, C., 2009. Regularized formulation of the variational brittle fracture with unilateral contact: Numerical experiments. J. Mech. Phys. Solids 57 (8), 12091229 . 
Bažant, Z., 1976. Instability, ductility and size effect in strain softening concrete. J. Eng. Mech. $102(2), 331-344$.

Bažant, Z., Zubelewicz, A., 1988. Strain-softening bar and beam: Exact non-local solution. Int. J. Solids Struct. 24 (7), $659-673$.

Bažant, Z. P., Pijaudier-Cabot, G., 1989. Measurement of characterisric length of non local continuum. J. Eng. Mech. 115 (4), 755-767.

Benallal, A., Billardon, R., Geymonat, G., 1993. Bifurcation and localization in rate independent materials. In: Nguyen, Q. (Ed.), C.S.I.M Lecture Notes on Bifurcation and Stability of Dissipative Systems. Vol. 190. Springer-Verlag, pp. 1-44.

Benallal, A., Marigo, J.-J., 2007. Bifurcation and stability issues in gradient theories. Model. Simul. Mater. Sci. Eng. 15, S283-S295.

Bourdin, B., 2007. Numerical implementation of the variational formulation of brittle fracture. Interfaces Free Bound. 9 (411-430).

Bourdin, B., Francfort, G., Marigo, J.-J., 2008. The variational approach to fracture. J. Elast. $91,1-148$.

Brezis, H., 1983. Analyse Fonctionnelle : Théorie et Applications. Masson, Paris.

Comi, C., 1999. Computational modelling of gradient-enhanced damage in quasi-brittle materials. Mech. Cohesive-Frict. Mater. 4 (1), 17-36.

Comi, C., 2001. A non-local model with tension and compression damage mechanisms. Eur. J. Mech. A/Solids 20 (1), $1-22$.

Comi, C., Perego, U., 2001. Fracture energy based bi-dissipative damage model for concrete. Int. J. Solids Struct. 38 (36-37), 6427-6454.

Dacorogna, B., 1992. Introduction au calcul des variations. Cahiers Mathématiques de l'École Polytechnique Fédérale de Lausanne. Editions Presses Polytechniques et Universitaires Romandes.

Frémond, M., Nedjar, B., 1996. Damage, gradient of damage and principle of virtual power. Int. J. Solids Struct. 33 (8), 1083 - 1103.

Geers, M., Peerlings, R., Brekelmans, W., de Borst, R., 2000. Phenomenological nonlocal approaches based on implicit gradient-enhanced damage. Acta Mech. 144 (1-2), 1-15.

Hill, R., 1958. A general theory for uniqueness and stability for elastic plastic solids. J. Mech. Phys. Solids 6, 236-249.

Hill, R., 1962. Acceleration waves in solids. J. Mech. Phys. Solids 10 (1), 1-16. 
Lorentz, E., Andrieux, S., 1999. A variational formulation of nonlocal damage models. Int. J. Plast. 15, 119-138.

Lorentz, E., Andrieux, S., 2003. Analysis of non-local models through energetic formulations. Int. J. Solids Struct. 40 (12), 2905-2936.

Lorentz, E., Benallal, A., 2005. Gradient constitutive relations: numerical aspects and applications to gradient damage. Comput. Methods Appl. Mech. Eng. 194, 5191-5220.

Marigo, J.-J., 1981. Formulation d'une loi d'endommagement d'un matériau élastique. C. R. Acad. Sci. Paris Sér. II 292 (19), 1309-1312.

Marigo, J.-J., 1989. Constitutive relations in plasticity, damage and fracture mechanics based on a work property. Nucl. Eng. Des. 114, 249-272.

Marigo, J.-J., 2000. L'endommagement et la rupture : hier, aujourd'hui et demain, Lecture notes of the IPSI course "Comportements non linéaires des matériaux" (Paris, mars 2000).

Mazars, J., 1986. A model of a unilateral elastic damageable material and its application to concrete. In: Fracture Toughness and Fracture Energy of Concrete. Elsevier, New York.

Mazars, J., Ramtani, S., Berthaud, Y., 1988. An experimental procedure to delocalize tensile failure and to indentify the unilateral effect of tensile damage. In: Mazars, J., Bažant, Z. P. (Eds.), Workshop on strain localization and size effect due to damage and cracking. Elsevier, Northwestern, pp. 581-587.

Mielke, A., 2005. Evolution of rate-independent systems. In: Evolutionary equations. Vol. II of Handb. Differ. Equ. Elsevier/North-Holland, Amsterdam, pp. 461-559.

Nguyen, Q. S., 1987. Bifurcation and postbifurcation analysis in plasticity and brittle fracture. J. Mech. Phys. Solids 35, 303-324.

Nguyen, Q. S., 2000. Stability and Nonlinear Solid Mechanics. Wiley \& Son, London.

Peerlings, R., Geers, M., deBorst, R., Brekelmans, W., 2001. A critical comparison of nonlocal and gradient-enhanced softening continua. Int. J. Solids Struct. 38 (44-45), 7723-7746.

Pham, K., Amor, H., Marigo, J.-J., Maurini, C., 2010. Gradient damage models and their use to approximate brittle fracture. Int. J. Damage Mech. DOI: 10.1177/1056789510386852, published online 25 November 2010.

Pham, K., Marigo, J.-J., 2009a. Construction and analysis of localized responses for gradient damage models in a 1d setting. Vietnam J. Mech. 31 (3-4), 233-246.

Pham, K., Marigo, J.-J., 2009b. Stability of non localized responses for damaging materials. Vietnam J. Mech. 30 (4), 1-11. 
Pham, K., Marigo, J.-J., 2010a. Approche variationnelle de l'endommagement : I. Les concepts fondamentaux. C. R. Mécanique 338 (4), 191-198.

Pham, K., Marigo, J.-J., 2010b. Approche variationnelle de l'endommagement : II. Les modèles à gradient. C. R. Mécanique 338 (4), 199-206.

Pijaudier-Cabot, G., Bazant, Z., Oct. 1987. Nonlocal damage theory. J. Eng. Mech. 113 (10), $1512-1533$.

Pijaudier-Cabot, G., Benallal, A., 1993. Strain localization and bifurcation in a nonlocal continuum. Int. J. Solids Struct. 30 (13), 1761-1775.

\section{Appendix A. Minimization of the Rayleigh ratio}

Let $\mathrm{a}>0, \mathrm{~b}>0$ and $\mathrm{c} \neq 0$ three given real numbers. Let $\mathcal{V}=H_{0}^{1}(0,1) \times H^{1}(0,1)$ be the Sobolev space equipped with its natural norm and let us define the Rayleigh ratio $\mathcal{R}$ on $\mathcal{V}$ by

$$
\mathcal{R}(v, \beta)= \begin{cases}\frac{\int_{0}^{1}\left(\mathrm{a} \beta^{\prime}(x)^{2}+\mathrm{b}\left(v^{\prime}(x)-\mathrm{c} \beta(x)\right)^{2}\right) d x}{\int_{0}^{1} \beta(x)^{2} d x} & \text { if } \beta \neq 0 \\ +\infty & \text { if } \beta=0\end{cases}
$$

Let us show that $\mathcal{R}$ is positive on $\mathcal{V}$. The Rayleigh ratio is non negative because a $>0$ and b $>0$. In order that $\mathcal{R}=0$, we should have $\beta(x)=$ const and $v^{\prime}=\mathrm{c}$ const. In such a case, since $v(0)=v(1)=0$, we should have $0=\int_{0}^{1} v^{\prime}(x) d x=\mathrm{c}$ const and hence $\beta=0$ since $\mathrm{c} \neq 0$. But $\beta=0$ is not allowed and hence $\mathcal{R}(v, \beta)>0$ for every $(v, \beta) \in \mathcal{V}$.

We want to calculate the minimum of $\mathcal{R}$ over $\mathcal{V}$ and its minimum over the convex subset $\mathcal{V} \cap\{\beta \geq 0\}$. In both cases the minimum exists (in the sense that it is reached by an admissible pair $\left.\left(v_{*}, \beta_{*}\right)\right)$ by virtue of the compactness of the injection of $H^{1}$ into $L^{2}$ and by the weak lower semi-continuity of a semi-norm. We can eliminate the field $v$ by minimizing $\mathcal{R}$ with respect to $v$ at given $\beta$. By standard arguments of calculus of variations, we immediately deduce that the minimizer $v_{*}$ is such that $v_{*}^{\prime \prime}=\mathrm{c} \beta^{\prime}$ and hence that there exists a constant $d$ such that $\mathrm{c} \beta(x)-v_{*}^{\prime}(x)=d$ for all $x$ in $(0,1)$. Since $v_{*}(0)=v_{*}(1)=0$, integrating the previous relation over $(0,1)$ gives $d=c\langle\beta\rangle$ and

$$
v_{*}^{\prime}(x)=\mathrm{c} \beta(x)-\mathrm{c}\langle\beta\rangle, \quad\langle\beta\rangle:=\int_{0}^{1} \beta(x) d x .
$$


Inserting this relation into the definition of $\mathcal{R}$, the Rayleigh ratio becomes the following functional defined on $H^{1}(0,1)$ :

$$
\hat{\mathcal{R}}(\beta)= \begin{cases}\frac{\mathrm{a} \int_{0}^{1} \beta^{\prime}(x)^{2} d x+\mathrm{bc}^{2}\left(\int_{0}^{1} \beta(x) d x\right)^{2}}{\int_{0}^{1} \beta(x)^{2} d x} & \text { if } \beta \neq 0 \\ +\infty & \text { if } \beta=0\end{cases}
$$

Appendix A.1. Minimization of $\hat{\mathcal{R}}$ over $H^{1}(0,1)$

Let $\beta_{*}$ be a minimizer and $\mathrm{R}=\hat{\mathcal{R}}\left(\beta_{*}\right)>0$. The directional derivative of $\hat{\mathcal{R}}$ at $\beta_{*}$ must vanish in every direction $\beta \in H^{1}(0,1)$ and hence

$$
\mathrm{a} \int_{0}^{1} \beta_{*}^{\prime}(x) \beta^{\prime}(x) d x+\mathrm{bc}^{2}\left\langle\beta_{*}\right\rangle \int_{0}^{1} \beta(x) d x=\mathrm{R} \int_{0}^{1} \beta_{*}(x) \beta(x) d x, \quad \forall \beta \in H^{1}(0,1)
$$

By standard arguments of calculus of variations, we immediately deduce that $\beta_{*}$ is such that

$$
\mathrm{a} \beta_{*}^{\prime \prime}(x)+\mathrm{R} \beta_{*}(x)=\mathrm{bc}^{2}\left\langle\beta_{*}\right\rangle, \quad \forall x \in(0,1)
$$

with the natural boundary conditions $\beta_{*}^{\prime}(0)=\beta_{*}^{\prime}(1)=0$. This second order ordinary differential equation with constant coefficients (and constant second member) has for general solution

$$
\beta_{*}(x)=A \cos \sqrt{\frac{\mathrm{R}}{\mathrm{a}}} x+B \sin \sqrt{\frac{\mathrm{R}}{\mathrm{a}}} x+\frac{\mathrm{bc}^{2}}{\mathrm{R}}\left\langle\beta_{*}\right\rangle .
$$

The natural boundary conditions give $0=B$ and $0=A \sin \sqrt{\mathrm{R} / \mathrm{a}}$. Moreover, integrating (A.1) over $(0,1)$ gives the equation for the integral of $\beta_{*}:\left(\mathrm{R}-\mathrm{bc}^{2}\right)\left\langle\beta_{*}\right\rangle=0$. Accordingly, there exists two possibilities:

1. Either $A=0$. In such a case, $\beta_{*}$ is constant and since this constant cannot be 0 we get $\mathrm{R}=\mathrm{bc}^{2}$

2. Or $A \neq 0$. In such a case, $\sin \sqrt{\mathrm{R} / \mathrm{a}}=0$ gives $\mathrm{R}=\pi^{2} \mathrm{a}$.

Finally we have obtained the following result

Proposition A.1. The minimum of the Rayleigh ratio $\mathcal{R}$ over $\mathcal{V}$ is equal to the minimum of $\mathrm{bc}^{2}$ and $\pi^{2} \mathrm{a}$ :

$$
\min _{\mathcal{V}} \mathcal{R}=\min \left\{\mathrm{bc}^{2}, \pi^{2} \mathrm{a}\right\}
$$

Moreover the eigenspace of minimizers is given by

$$
\beta_{*}(x)=C+A \cos \pi x, \quad v_{*}(x)=\frac{\mathrm{c} A}{\pi} \sin \pi x
$$

where $A=0$ if $\mathrm{bc}^{2}<\pi^{2} \mathrm{a}, C=0$ if $\mathrm{bc}^{2}>\pi^{2} \mathrm{a}$ and, $A$ and $C$ are arbitrary real numbers otherwise. 
Appendix A.2. Minimization of $\hat{\mathcal{R}}$ over $\mathcal{H}_{+}=H^{1}(0,1) \cap\{\beta \geq 0\}$

Let $\beta_{*}$ be a minimizer and $\mathrm{R}=\hat{\mathcal{R}}\left(\beta_{*}\right)>0$. Since $\mathcal{H}_{+}$is not a linear space, but only a convex set, the minimizer has to satisfy the variational inequality $\hat{\mathcal{R}}^{\prime}\left(\beta_{*}\right)(\beta) \geq 0, \forall \beta \in \mathcal{H}_{+}$which reads as

$$
\mathrm{a} \int_{0}^{1} \beta_{*}^{\prime}(x) \beta^{\prime}(x) d x+\mathrm{bc}^{2}\left\langle\beta_{*}\right\rangle \int_{0}^{1} \beta(x) d x \geq \mathrm{R} \int_{0}^{1} \beta_{*}(x) \beta(x) d x, \quad \forall \beta \in \mathcal{H}_{+} .
$$

The inequality becomes an equality when $\beta=\beta_{*}$.

It is then possible to prove that the minimizer is in $H^{2}(0,1)$ (and hence is continuously differentiable) but we omit its technical proof and assume that this smoothness property holds. Accordingly, after an integration by parts, (A.2) becomes

$$
0 \geq \int_{0}^{1}\left(\mathrm{a} \beta_{*}^{\prime \prime}(x)+\mathrm{R} \beta_{*}(x)-\mathrm{bc}^{2}\left\langle\beta_{*}\right\rangle\right) \beta(x) d x-\mathrm{a} \beta_{*}^{\prime}(1) \beta(1)+\mathrm{a} \beta_{*}^{\prime}(0) \beta(0), \quad \forall \beta \in \mathcal{H}_{+}
$$

and by standard arguments we get

$$
\mathrm{a} \beta_{*}^{\prime \prime}+\mathrm{R} \beta_{*} \leq \mathrm{bc}^{2}\left\langle\beta_{*}\right\rangle \quad \text { in }(0,1), \quad \beta_{*}^{\prime}(1) \geq 0, \quad \beta_{*}^{\prime}(0) \leq 0 .
$$

But since the equality holds in (A.2) when $\beta=\beta_{*}$ and since $\beta_{*} \geq 0$ we have also

$$
\left(\mathrm{a} \beta_{*}^{\prime \prime}+\mathrm{R} \beta_{*}-\mathrm{bc}^{2}\left\langle\beta_{*}\right\rangle\right) \beta_{*}=0 \quad \text { in }(0,1), \quad \beta_{*}^{\prime}(1) \beta_{*}(1)=\beta_{*}^{\prime}(0) \beta(0)=0 .
$$

Therefore, if $\beta_{*}(0)>0$, then $\beta_{*}^{\prime}(0)=0$. If $\beta_{*}(0)=0$, since $\beta_{*} \geq 0$, we must have $\beta_{*}^{\prime}(0) \geq 0$. But, by virtue of $\left(\right.$ A.3), we must also have $\beta_{*}^{\prime}(0) \leq 0$. Hence, in all cases, $\beta_{*}^{\prime}(0)=0$. Similarly, in all cases, $\beta_{*}^{\prime}(1)=0$. Accordingly, the minimizer is an element of $H^{2}(0,1)$ which satisfies

$$
\beta_{*} \geq 0, \quad \mathrm{a} \beta_{*}^{\prime \prime}+\mathrm{R} \beta_{*}-\mathrm{bc}^{2}\left\langle\beta_{*}\right\rangle \leq 0, \quad\left(\mathrm{a} \beta_{*}^{\prime \prime}+\mathrm{R} \beta_{*}-\mathrm{bc} \mathrm{c}^{2}\left\langle\beta_{*}\right\rangle\right) \beta_{*}=0, \quad \beta_{*}^{\prime}(0)=\beta_{*}^{\prime}(1)=0
$$

Let us denote by $\operatorname{supp} \beta_{*}$ the support of $\beta_{*}$, i.e. $\operatorname{supp} \beta_{*}=\left\{x \in(0,1): \beta_{*}(x)>0\right\}$. Since $\beta_{*} \neq 0$ and $\beta_{*}$ is continuous, $\operatorname{supp} \beta_{*}$ is a not empty open set. Let $I=\left(x_{m}, x_{M}\right)$ be a connected component of $\operatorname{supp} \beta_{*}$. At the ends of the interval, we necessarily have $\beta_{*}^{\prime}\left(x_{m}\right)=\beta_{*}^{\prime}\left(x_{M}\right)=0$. Indeed the equality holds at 0 and 1 . If $x_{m}>0$ (resp. $\left.x_{M}<1\right)$ then, by definition of $I$, we have $\beta_{*}\left(x_{m}\right)=0$ (resp. $\beta_{*}\left(x_{M}\right)=0$ ) and, since $\beta_{*}$ is non negative and differentiable, $\beta_{*}^{\prime}$ must vanish at the points where $\beta_{*}$ vanishes.

Accordingly, $\beta_{*}$ must satisfy

$$
\begin{array}{ccc}
\beta_{*}(x)>0, \quad \mathrm{a} \beta_{*}^{\prime \prime}(x)+\mathrm{R} \beta_{*}(x)=\mathrm{bc}^{2}\left\langle\beta_{*}\right\rangle, \quad \forall x \in\left(x_{m}, x_{M}\right) \\
\beta_{*}^{\prime}\left(x_{m}\right)=0, \quad \beta_{*}^{\prime}\left(x_{M}\right)=0 \\
\beta_{*}\left(x_{m}\right)=0 \quad \text { if } \quad x_{m}>0, \quad \beta_{*}\left(x_{M}\right)=0 \quad \text { if } \quad x_{M}<1
\end{array}
$$

Let us examine the different possibilities 
1. Case where $\beta_{*}\left(x_{m}\right)>0$ and $\beta_{*}\left(x_{M}\right)>0$. Then $I=(0,1)$. By easy calculations, we find $\beta_{*}(x)=\frac{\mathrm{bc}^{2}}{\mathrm{R}}\left\langle\beta_{*}\right\rangle+A \cos \sqrt{\mathrm{R} / \mathrm{a}} x$ with $A \sin \sqrt{\mathrm{R} / \mathrm{a}}=0$. Accordingly, there exist two subcases:

(a) If $A=0$, then $\beta_{*}$ is a positive constant and $\mathrm{R}=\mathrm{bc}^{2}$.

(b) If $A \neq 0$, then there exists $n \in \mathbb{N}_{*}$ such that $\mathrm{R}=n^{2} \pi^{2}$ a. From the definition of $\left\langle\beta_{*}\right\rangle$ we get $\left\langle\beta_{*}\right\rangle=\frac{\mathrm{bc}^{2}}{\mathrm{R}}\left\langle\beta_{*}\right\rangle$. But since $\left\langle\beta_{*}\right\rangle>0$, we obtain also $\mathrm{R}=\mathrm{bc}^{2}$.

Finally, in such a case, we have

$$
\mathrm{R}=\mathrm{bc}^{2}, \quad \beta_{*}(x)=C+A_{n} \cos n \pi x \quad \text { with } \quad C>0, \quad\left|A_{n}\right| \begin{cases}=0 & \text { if } \mathrm{bc}^{2} \neq n^{2} \pi^{2} \mathrm{a} \\ <C & \text { if } \mathrm{bc}^{2}=n^{2} \pi^{2} \mathrm{a}\end{cases}
$$

2. Case where $\beta_{*}\left(x_{m}\right)>0$ and $\beta_{*}\left(x_{M}\right)=0$. Then $I=(0, \mathrm{D})$ with $0<\mathrm{D} \leq 1$. We have $\beta_{*}(x)=\frac{\mathrm{bc}^{2}}{\mathrm{R}}\left\langle\beta_{*}\right\rangle+A \cos \sqrt{\mathrm{R} / \mathrm{a}} x$ in $(0, \mathrm{D})$ and the boundary conditions at $\mathrm{D}$ read as

$$
\frac{\mathrm{bc}^{2}}{\mathrm{R}}\left\langle\beta_{*}\right\rangle+A \cos \sqrt{\mathrm{R} / \mathrm{a}} \mathrm{D}=0, \quad A \sin \sqrt{\mathrm{R} / \mathrm{a}} \mathrm{D}=0
$$

The constant $A$ cannot vanishes, because we should have $\left\langle\beta_{*}\right\rangle=0$ what is impossible. Hence $\mathrm{R}=n^{2} \pi^{2} \mathrm{a} / \mathrm{D}^{2}$ with $n \in \mathbb{N}_{*}$ and $\frac{\mathrm{bc}^{2}}{\mathrm{R}}\left\langle\beta_{*}\right\rangle+(-1)^{n} A=0$. But since $\beta_{*}>0$ in [0, D), we must take $n=1$ and hence

$$
\mathrm{R}=\frac{\pi^{2} \mathrm{a}}{\mathrm{D}^{2}}, \quad \beta_{*}(x)=\frac{\mathrm{bc}^{2}}{\pi^{2} \mathrm{a}} \mathrm{D}^{2}\left\langle\beta_{*}\right\rangle\left(1+\cos \pi \frac{x}{\mathrm{D}}\right), \quad \forall x \in[0, \mathrm{D}] .
$$

Calculating the integral of $\beta_{*}$ over $I$ gives $\int_{0}^{\mathrm{D}} \beta_{*}(x) d x=\frac{\mathrm{bc}^{2}}{\pi^{2} \mathrm{a}} \mathrm{D}^{3}\left\langle\beta_{*}\right\rangle$. Let us consider two subcases corresponding to the partitioning of the support of $\beta_{*}$ :

(a) If $I$ is the unique connected component of $\operatorname{supp} \beta_{*}$, then $\beta_{*}=0$ in $[\mathrm{D}, 1]$ and $\int_{0}^{\mathrm{D}} \beta_{*}(x) d x=$ $\left\langle\beta_{*}\right\rangle>0$. Therefore $\mathrm{D}^{3}=\frac{\pi^{2} \mathrm{a}}{\mathrm{bc} c^{2}}$ and $\mathrm{R}=\left(\pi^{2} \mathrm{a}\right)^{1 / 3}\left(\mathrm{bc}^{2}\right)^{2 / 3}$. The condition $\mathrm{D} \leq 1$ requires that $\pi^{2} \mathrm{a} \leq \mathrm{bc}^{2}$.

(b) If there exists another connected component, then $\int_{0}^{D} \beta_{*}(x) d x<\left\langle\beta_{*}\right\rangle$, $\mathrm{D}^{3}<\frac{\pi^{2} \mathrm{a}}{\mathrm{bc}^{2}}$ and $\left.\mathrm{R}>\left(\pi^{2} \mathrm{a}\right)^{1 / 3}(\mathrm{bc})^{2}\right)^{2 / 3}$.

3. Case where $\beta_{*}\left(x_{m}\right)=0$ and $\beta_{*}\left(x_{M}\right)>0$. It is a case symmetric to the previous one. We obtain the same results by changing $x$ by $1-x$.

4. Case where $\beta_{*}\left(x_{m}\right)=\beta_{*}\left(x_{M}\right)=0$. Let us set $x_{i}=\left(x_{M}+x_{m}\right) / 2$ and $\mathrm{D}=\left(x_{M}-x_{m}\right) / 2$. Then, after calculations similar to those of the two previous cases we get

$$
\mathrm{R}=\frac{\pi^{2} \mathrm{a}}{\mathrm{D}^{2}}, \quad \beta_{*}(x)=\frac{\mathrm{bc}^{2}}{\pi^{2} \mathrm{a}} \mathrm{D}^{2}\left\langle\beta_{*}\right\rangle\left(1+\cos \pi \frac{x-x_{i}}{\mathrm{D}}\right), \quad \forall x \in\left[x_{i}-\mathrm{D}, x_{i}+\mathrm{D}\right] .
$$

Moreover $\int_{I} \beta_{*}(x) d x=\frac{\mathrm{bc}^{2}}{\pi^{2} \mathrm{a}} 2 \mathrm{D}^{3}\left\langle\beta_{*}\right\rangle$ and we still consider two subcases corresponding to the partitioning of the support of $\beta_{*}$ :

(a) If $I$ is the unique connected component of $\operatorname{supp} \beta_{*}$, then $\int_{I} \beta_{*}(x) d x=\left\langle\beta_{*}\right\rangle>0$. Therefore $\mathrm{D}^{3}=\frac{\pi^{2} \mathrm{a}}{2 \mathrm{bc} c^{2}}$ and $\mathrm{R}=\left(\pi^{2} \mathrm{a}\right)^{1 / 3}\left(2 \mathrm{bc}^{2}\right)^{2 / 3}$. The condition $2 \mathrm{D} \leq 1$ requires that $4 \pi^{2} \mathrm{a} \leq \mathrm{bc}^{2}$. 
(b) If there exists another connected component, then $\int_{I} \beta_{*}(x) d x<\left\langle\beta_{*}\right\rangle$, $\mathrm{D}^{3}<\frac{\pi^{2} \mathrm{a}}{2 \mathrm{bc} c^{2}}$ and $\mathrm{R}>\left(\pi^{2} \mathrm{a}\right)^{1 / 3}\left(2 \mathrm{bc} c^{2}\right)^{2 / 3}$.

Comparing the different cases, we see that if $\pi^{2} a>b c^{2}$ then the subcase 1a gives the minimal value for the Rayleigh ratio, while if $\pi^{2} a<b^{2}$ then the minimal value is obtained in subcase $2 \mathrm{a}$ (or symmetrically in subcase $3 \mathrm{a}$ ) and the support of the minimizer consists in a unique connected component starting at $x=0$ or finishing at $x=1$. Specifically, we have

Proposition A.2. The minimization of the Rayleigh ratio $\hat{\mathcal{R}}$ over $\mathcal{H}_{+}$leads to

1. If $\pi^{2} \mathrm{a}>\mathrm{bc}^{2}$, then $\min _{\mathcal{H}_{+}} \hat{\mathcal{R}}=\mathrm{bc}^{2}$ and the minimizers are $\beta_{*}(x)=C>0$.

2. If $\pi^{2} \mathrm{a}=\mathrm{bc}^{2}$, then $\min _{\mathcal{H}_{+}} \hat{\mathcal{R}}=\mathrm{bc}^{2}$ and the minimizers are $\beta_{*}(x)=C+A \cos \pi x$ with $C>0$ and $|A| \leq C$.

3. If $\pi^{2} \mathrm{a}<\mathrm{bc}^{2}$, then $\min _{\mathcal{H}_{+}} \hat{\mathcal{R}}=\left(\pi^{2} \mathrm{a}\right)^{1 / 3}\left(\mathrm{bc}^{2}\right)^{2 / 3}$ and the minimizers are

$$
\beta_{*}(x)=\left\{\begin{array}{ll}
C\left(1+\cos \pi \frac{x}{\mathrm{D}}\right), & \text { if } x \in(0, \mathrm{D}) \\
0 & \text { otherwise }
\end{array} \quad \text { and } \quad \tilde{\beta}_{*}(x)=\beta_{*}(1-x)\right.
$$

where $C$ is an arbitrary positive constant and $\mathrm{D}^{3}=\frac{\pi^{2} \mathrm{a}}{\mathrm{bc}^{2}}$.

\section{Appendix B. The bifurcation problem}

Many arguments used in this section are those developed in the previous one. Hence we merely refer to that section when we need such arguments. Let $\mathrm{a}, \mathrm{b}$ and $\mathrm{c}$ three given positive real numbers such that $1<\pi \sqrt{\mathrm{a}} \mathrm{bc}^{2} \leq \mathrm{bc}^{2}$ and let $\mathcal{H}_{+}=\left\{\beta \in H^{1}(0,1): \beta \geq 0\right\}$. We consider the following variational problem

Find $\dot{\alpha} \in \mathcal{H}_{+}$such that $\forall \beta \in H^{1}(0,1)$

$$
\mathrm{a} \int_{0}^{1} \dot{\alpha}^{\prime}\left(\beta^{\prime}-\dot{\alpha}^{\prime}\right) d x+\mathrm{bc}^{2}\langle\dot{\alpha}\rangle \int_{0}^{1}(\beta-\dot{\alpha}) d x-\int_{0}^{1} \dot{\alpha}(\beta-\dot{\alpha}) d x \geq \mathrm{bc} \int_{0}^{1}(\beta-\dot{\alpha}) d x
$$

where $\langle\dot{\alpha}\rangle=\int_{0}^{1} \dot{\alpha} d x$. This problem admits the constant solution $\dot{\alpha}(x)=\mathrm{bc} /\left(\mathrm{bc}^{2}-1\right), \forall x \in[0,1]$ and the issue is to know whether the solution is unique.

Even though it is possible to prove that any solution belongs to $H^{2}(0,1)$ (and hence is continuously differentiable), we omit the technical proof of this smoothness property and assume that it holds. Accordingly, after an integration by parts, (B.1) becomes

$$
0 \geq \int_{0}^{1}\left(\mathrm{a} \dot{\alpha}^{\prime \prime}+\dot{\alpha}+\mathrm{bc}(1-\mathrm{c}\langle\dot{\alpha}\rangle)\right)(\beta-\dot{\alpha}) d x-\mathrm{a} \dot{\alpha}^{\prime}(1)(\beta(1)-\dot{\alpha}(1))+\mathrm{a} \dot{\alpha}^{\prime}(0)(\beta(0)-\dot{\alpha}(0)) .
$$


By standard arguments, we first obtain that $\dot{\alpha}$ must satisfy

$$
\dot{\alpha} \geq 0, \quad \mathrm{a} \dot{\alpha}^{\prime \prime}+\dot{\alpha}+\mathrm{bc}(1-\mathrm{c}\langle\dot{\alpha}\rangle) \leq 0, \quad \dot{\alpha}\left(\mathrm{a} \dot{\alpha}^{\prime \prime}+\dot{\alpha}+\mathrm{bc}(1-\mathrm{c}\langle\dot{\alpha}\rangle)\right)=0 \quad \text { a.e. in }(0,1)
$$

with the boundary conditions

$$
\dot{\alpha}(0) \geq 0, \quad \dot{\alpha}^{\prime}(0) \leq 0, \quad \dot{\alpha}(0) \dot{\alpha}^{\prime}(0)=0, \quad \dot{\alpha}(1) \geq 0, \quad \dot{\alpha}^{\prime}(1) \geq 0, \quad \dot{\alpha}(1) \dot{\alpha}^{\prime}(1)=0 .
$$

By the same arguments as those used in the previous section, we show that the boundary conditions above imply that $\dot{\alpha}^{\prime}(0)=\dot{\alpha}^{\prime}(1)=0$. Finally $\dot{\alpha}$ must be an element of $H^{2}(0,1)$ which satisfies (B.2) and the boundary conditions $\dot{\alpha}^{\prime}(0)=\dot{\alpha}^{\prime}(1)=0$.

Since bc $>0, \dot{\alpha}=0$ cannot be solution and hence supp $\dot{\alpha}$ is not empty. Let $I=\left(x_{m}, x_{M}\right)$ be a connected component of supp $\dot{\alpha}$. At the ends of the interval, we necessarily have $\dot{\alpha}^{\prime}\left(x_{m}\right)=$ $\dot{\alpha}^{\prime}\left(x_{M}\right)=0$, see Appendix A.2. Accordingly, $\dot{\alpha}$ must satisfy

$$
\begin{aligned}
\dot{\alpha}(x)>0, \quad \mathrm{a} \dot{\alpha}^{\prime \prime}(x)+\dot{\alpha}(x)=\mathrm{bc}^{2}\langle\dot{\alpha}\rangle-\mathrm{bc}, \quad \forall x \in\left(x_{m}, x_{M}\right) \\
\dot{\alpha}^{\prime}\left(x_{m}\right)=0, \quad \dot{\alpha}^{\prime}\left(x_{M}\right)=0 \\
\dot{\alpha}\left(x_{m}\right)=0 \quad \text { if } \quad x_{m}>0, \quad \dot{\alpha}\left(x_{M}\right)=0 \quad \text { if } \quad x_{M}<1
\end{aligned}
$$

Let us examine the different possibilities

1. Case where $\dot{\alpha}\left(x_{m}\right)>0$ and $\dot{\alpha}\left(x_{M}\right)>0$. Then $I=(0,1)$. By easy calculations, we find $\dot{\alpha}(x)=\mathrm{bc}^{2}\langle\dot{\alpha}\rangle-\mathrm{bc}+A \cos x / \sqrt{\mathrm{a}}$ with $A \sin 1 / \sqrt{\mathrm{a}}=0$. Hence, there exists two subcases:

(a) If $A=0$, then $\dot{\alpha}$ is the constant solution bc/(bc $\left.\mathrm{bc}^{2}-1\right)$;

(b) If $A \neq 0$, then $\sin 1 / \sqrt{\mathrm{a}}=0$ requires that there exists $n \in \mathbb{N}_{*}$ such that $n^{2} \pi^{2} \mathrm{a}=1$. Integrating $\dot{\alpha}$ over $(0,1)$ gives $\langle\dot{\alpha}\rangle=\mathrm{bc} /\left(\mathrm{bc}^{2}-1\right)$. Finally, $\dot{\alpha}$ is given by

$$
\dot{\alpha}(x)=\frac{\mathrm{bc}}{\mathrm{bc}^{2}-1}+A \cos n \pi x, \quad|A|<\frac{\mathrm{bc}}{\mathrm{bc}^{2}-1}, \quad n \pi \sqrt{\mathrm{a}}=1, \quad n \in \mathbb{N}_{*}
$$

where the value of $A$ is only limited by the condition $\dot{\alpha}>0$ in $[0,1]$.

2. Case where $\dot{\alpha}\left(x_{m}\right)>0$ and $\dot{\alpha}\left(x_{M}\right)=0$. Then $I=(0, \mathrm{D})$ with $0<\mathrm{D} \leq 1$. We get $\dot{\alpha}(x)=\mathrm{bc}^{2}\langle\dot{\alpha}\rangle-\mathrm{bc}+A \cos x / \sqrt{\mathrm{a}}$ in $[0, \mathrm{D}]$ and the boundary conditions at $\mathrm{D}$ read as

$$
\mathrm{bc}^{2}\langle\dot{\alpha}\rangle-\mathrm{bc}+A \cos \frac{\mathrm{D}}{\sqrt{\mathrm{a}}}=0, \quad A \sin \frac{\mathrm{D}}{\sqrt{\mathrm{a}}}=0
$$

The case $A=0$ is not possible, because that should lead to $\dot{\alpha}=0$ in $[0, \mathrm{D})$, what is not allowed. Therefore $A \neq 0$ and $\mathrm{D}=n \pi \sqrt{\mathrm{a}}$ with $n \in \mathbb{N}_{*}$. Hence $\dot{\alpha}(x)=\left(\mathrm{bc}^{2}\langle\dot{\alpha}\rangle-\mathrm{bc}\right)\left(1-(-1)^{n} \cos n \pi x / \mathrm{D}\right)$ in $[0, \mathrm{D}]$. But since $\dot{\alpha}>0$ in $[0, \mathrm{D})$, we must take $n=1$ and finally we have

$$
\mathrm{D}=\pi \sqrt{\mathrm{a}}, \quad \dot{\alpha}(x)=\left(\mathrm{bc}^{2}\langle\dot{\alpha}\rangle-\mathrm{bc}\right)\left(1+\cos \pi \frac{x}{\mathrm{D}}\right) \quad \text { in } \quad[0, \mathrm{D}] .
$$

It remains to determine $\langle\dot{\alpha}\rangle$ which depends on the other possible connected components of supp $\dot{\alpha}$ and to check that $\dot{\alpha} \geq 0$ everywhere. 
3. Case where $\dot{\alpha}\left(x_{m}\right)=0$ and $\dot{\alpha}\left(x_{M}\right)>0$. It is a case symmetric to the previous one. We obtain the same results by changing $x$ by $1-x$.

4. Case where $\dot{\alpha}\left(x_{m}\right)=\dot{\alpha}\left(x_{M}\right)=0$. We set $x_{i}=\left(x_{M}+x_{m}\right) / 2$ and $\mathrm{D}=\left(x_{M}-x_{m}\right) / 2$. Then, after calculations similar to those of the two previous cases we get

$$
\mathrm{D}=\pi \sqrt{\mathrm{a}}, \quad \dot{\alpha}(x)=\left(\mathrm{bc}^{2}\langle\dot{\alpha}\rangle-\mathrm{bc}\right)\left(1+\cos \frac{x-x_{i}}{\sqrt{\mathrm{a}}}\right) \quad \text { in } \quad\left[x_{i}-\pi \sqrt{\mathrm{a}}, x_{i}+\pi \sqrt{\mathrm{a}}\right] .
$$

This case requires that $2 \pi \sqrt{\mathrm{a}} \leq 1$. When this condition is satisfied, $x_{i}$ can be arbitrarily chosen in $[\pi \sqrt{\mathrm{a}}, 1-\pi \sqrt{\mathrm{a}}]$.

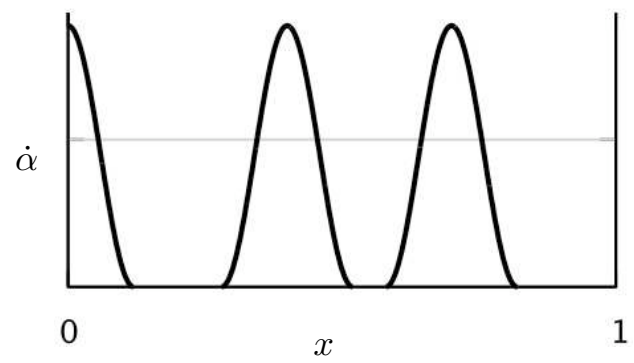

Figure B.8: Example of a non constant solution of the bifurcation problem where $\mathrm{bc}^{2}=12, \pi \sqrt{\mathrm{a}}=0.12$ and $n=5$. The solution is made of one half-sinusoid and two sinusoids. The gray line represents the constant solution.

Comparing the different cases, it appears that there exists a solution other than the constant one when $n^{2} \pi^{2} \mathrm{a}=1$ for some $n \in \mathbb{N}_{*}$. Otherwise, any solution must be a combination of cases 2, 3 and 4 above. The profile of $\dot{\alpha}$ is an half-sinusoid in cases 2 and 3 whereas the profile is a sinusoid in case 4 , but in each case the amplitude and the length of the sinusoids are the same. Accordingly, let us define $N \in \mathbb{N}_{*}$ such that $N \pi \sqrt{\mathrm{a}} \leq 1<(N+1) \pi \sqrt{\mathrm{a}}$. (Since $\pi \sqrt{\mathrm{a}} \mathrm{bc}^{2}>1$, we have $1 \leq N<\mathrm{bc}^{2}$.) Let us consider a combination of half-sinusoids and sinusoids whose total length is $n \pi \sqrt{\mathrm{a}}$ with $1 \leq n \leq N$. Since the integral of $\dot{\alpha}$ over an half-sinusoid is equal to $\left(\mathrm{bc}^{2}\langle\dot{\alpha}\rangle-\mathrm{bc}\right) \pi \sqrt{\mathrm{a}}$, we get

$$
\dot{\alpha}(x)=\frac{\mathrm{bc}}{n \pi \sqrt{\mathrm{a}} \mathrm{bc}^{2}-1}\left(1+\cos \frac{x-x_{i}}{\sqrt{\mathrm{a}}}\right)
$$

on each connected component of $\operatorname{supp} \dot{\alpha}$, i.e. on the interval $\left|x-x_{i}\right| \leq \pi \sqrt{\mathrm{a}}$ with $x_{i}=0$ in case 2 and $x_{i}=1$ in case 3 . Since $\pi \sqrt{\mathrm{a}} \mathrm{bc}^{2}>1, \dot{\alpha} \geq 0$ everywhere and this solution is admissible. Thus, we are in a position to conclude

Proposition B.1. Under the conditions that the positive parameters $\mathrm{a}, \mathrm{b}$ and $\mathrm{c}$ are such that $1<\pi \sqrt{\mathrm{a}} \mathrm{bc}^{2} \leq \mathrm{bc}^{2}$, the variational problem (B.1) admits other solutions than the constant one. In particular, the half-sinusoids $\dot{\alpha}$ and $\dot{\alpha}_{*}$ given by

$$
\dot{\alpha}(x)=\left\{\begin{array}{ll}
\frac{\mathrm{bc}}{\pi \sqrt{\mathrm{a}} \mathrm{bc}^{2}-1}\left(1+\cos \frac{x}{\sqrt{\mathrm{a}}}\right) & \text { in }(0, \pi \sqrt{\mathrm{a}}) \\
0 & \text { otherwise }
\end{array}, \quad \dot{\alpha}_{*}(x)=\dot{\alpha}(1-x)\right.
$$


are such solutions. Other solutions exist when $\mathrm{bc}^{2}$ is sufficiently large and $\pi \sqrt{\mathrm{a}}$ is sufficiently small. All those solutions are made of half-sinusoids or sinusoids with same (half-)length $\pi \sqrt{a}$ and same amplitude $2 \mathrm{bc} /\left(n \pi \sqrt{\mathrm{a}} \mathrm{bc}^{2}-1\right)$ where $n$ is the total number of half-sinusoids, but the locations of the sinusoids remain essentially arbitrary, see Figure B.8. 